\title{
Black Hole Radiation, Greybody Factors, and Generalised Wick Rotation
}

Finnian Padraig Stott Gray

\section{VICTORIA UNIVERSITY OF WELLINGTON}

Te Whare Wānanga o te Ūpoko o te Ika a Māui

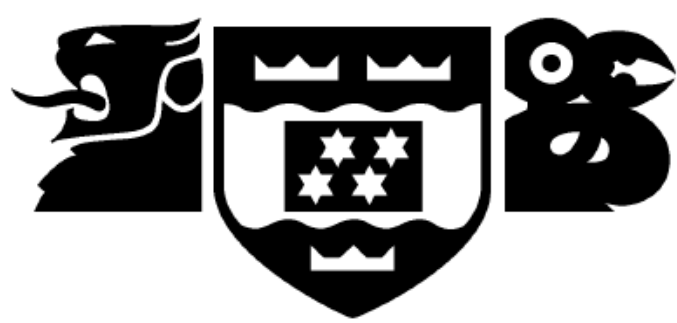

School of Mathematics and Statistics

Te Kura Mātai Tatauranga

\section{A thesis}

submitted to the Victoria University of Wellington in fulfilment of the requirements for the degree of

Master of Science

in Mathematics.

Victoria University of Wellington 



\begin{abstract}
In this thesis we look at the intersection of quantum field theory and general relativity. We focus on Hawking radiation from black holes and its implications. This is done on two fronts. In the first we consider the greybody factors arising from a Schwarzschild black hole. We develop a new way to numerically calculate these greybody factors using the transfer matrix formalism and the product calculus. We use this technique to calculate some of the relevant physical quantities and consider their effect on the radiation process.

The second front considers a generalisation of Wick rotation. This is motivated by the success of Wick rotation and Euclidean quantum field theory techniques to calculate the Hawking temperature. We find that, while an analytic continuation of the coordinates is not well defined and highly coordinate dependent, a direct continuation of the Lorentzian signature metric to Euclidean signature has promising results. It reproduces the Hawking temperature and is coordinate independent. However for consistency, we propose a new action for the Euclidean theory which cannot be simply the Euclidean Einstein-Hilbert action.
\end{abstract}





\section{Acknowledgements}

First and foremost I would like to thank my supervisor Prof. Matt Visser without whom the work conducted would not have been possible. His support, insights, and discussions have been invaluable in helping me learn all that I have over this past year. Thanks must also go to everyone in the VUW physics department for their teaching and support over my years here. I thank the Victoria Scholarship Office for supporting me financially via the Victoria Masters by Thesis Scholarship.

I would also like to thank the math grad students for such a welcoming and friendly environment.

Finally I thank my partner for her constant support and my parents for encouraging me and helping me to where I am. 



\section{Contents}

$\begin{array}{llr}1 & \text { Introduction } & 1\end{array}$

1.1 Arrangement of the Thesis . . . . . . . . . . . . . . . 2

2 Aspects of GR 5

2.1 Preliminaries . . . . . . . . . . . . . . . . . . . . 5

2.2 Curvature and Field Equations $\ldots \ldots \ldots$

2.3 Lagrangian Formulation . . . . . . . . . . . . . . . . . . . 15

\begin{tabular}{|lll}
3 & Aspects of QFT in Flat Spacetime & 21
\end{tabular}

3.1 Preliminaries and Canonical Quantisation . . . . . . . . . . 21

3.2 Path Integral Formulation . . . . . . . . . . . . . . . . . . 26

3.3 Wick Rotation and Euclidean Methods . . . . . . . . . . . . . 27

4 Applications of QFT in Curved Spacetime 31

4.1 Extension to Curved Space . . . . . . . . . . . . . . . . . 31

4.2 The Unruh Effect . . . . . . . . . . . . . . . . . . . . . . . . . 34

4.3 Hawking Radiation . . . . . . . . . . . . . . . . . . . . . . . . 37

5 Greybody Factors 43

5.1 Emission Rates from Schwarzschild Black Holes . . . . . . . . 43

5.2 Regge-Wheeler Equation . . . . . . . . . . . . . . . . . . . 45

5.3 Transfer Matrix Approach . . . . . . . . . . . . . . . . . . . . 52

5.4 Numerical Results . . . . . . . . . . . . . . . . . . . . . . . . . 59

5.5 Implications and Extensions . . . . . . . . . . . . . . . . . . 68

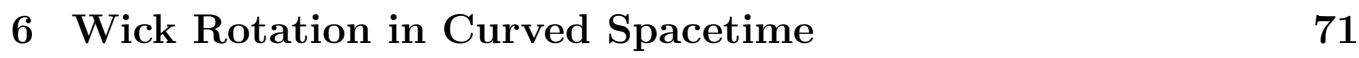

6.1 Euclidean Quantum Gravity . . . . . . . . . . . . . . . . . 71

6.2 Examples of Wick Rotation . . . . . . . . . . . . . . . . 73

6.3 Analytic Continuation of the Manifold . . . . . . . . . . . 77 
6.4 Analytic Continuation of the Metric . . . . . . . . . . . . . . 79

6.5 Open Problems and Further Work . . . . . . . . . . . . . . . . 90

\begin{tabular}{lll}
\hline 7 & Conclusions & 93
\end{tabular}

\section{List of Figures}

2.1 Penrose-Carter Diagram of Schwarzschild Black hole . . . . . 14

3.1 Wick Rotated Contour . . . . . . . . . . . . . . . . . . . . . 28

4.1 Penrose-Carter Diagram of Collapsing Body . . . . . . . . . . 38

$5.1 \quad$ Scalar Regge-Wheeler Potential . . . . . . . . . . . . . . . 47

5.2 Photon Regge-Wheeler Potential . . . . . . . . . . . . . . . . 49

5.3 Graviton Regge-Wheeler Potential . . . . . . . . . . . . . 50

5.4 Square Barrier Transmission Probabilities . . . . . . . . . . 62

5.5 Square Barrier Convergence Tests . . . . . . . . . . . . . 63

5.6 Greybody Convergence Tests . . . . . . . . . . . . . . . . . . . 64

5.7 Schwarzschild Greybody Factors . . . . . . . . . . . . . . . . . 65

5.8 Schwarzschild Emission Spectra . . . . . . . . . . . . . . 66

5.9 Schwarzschild Emission Cross-Section . . . . . . . . . . . . 67 


\section{Chapter 1}

\section{Introduction}

One of the great unresolved problems in physics is how to merge the fields of general relativity and quantum field theory. These theories are individually incredibly accurate, and have made many new predictions within their respective regimes of applicability, but have proved difficult to unify into one theory which describes both regimes. Quantum field theory (QFT) explains the microscopic world of fundamental particles moving at both high and low speeds, while general relativity encompasses, in the Einstein field equations (EFEs), the macroscopic world of the curvature of space-time due to the presence of massive objects.

The contrast of scales between the macroscopic and microscopic, and the relative weakness of gravity as compared to the other fundamental forces, makes experimental testing of quantum gravity theories extremely difficult. This means there is a variety of competing theories which as yet coexist. One starting point to uniting the two models is to consider quantum fields in a fixed curved spacetime background. This leads to interesting and suggestive effects such as the non-uniqueness of vacuum states and the creation of particles due to the background spacetimes. An example of this is Hawking radiation from black holes. This has a thermal spectrum modified by greybody factors resulting from particles scattering off the gravitational potential. These greybody factors are of importance when it comes to calculating detailed physical effects from the radiation process.

The calculation of many problems in (flat-space) QFT is made possible by a Wick rotation, in which time is analytically continued to an imaginary vari- 
able $t \rightarrow \tau=-i t$. This effectively changes the signature of the background Minkowski spacetime metric from Lorentzian to Euclidean and allows the development of a formalism for quantization via the path integral approach which is deeply related to quantum statistical mechanics. This leads to one route to the Hawking temperature for black holes, and completes the analogy between the laws of black hole mechanics and thermodynamics.

It is for this reason that Euclidean quantum gravity (EQG), in which the Lorentzian signature of spacetime is transformed into a standard Euclidean one, is suggested as a possible route to uniting general relativity and QFT. There is however, at this stage, no standard procedure by which to effect this signature change for an arbitrary spacetime. This stems from the nonexistence of a unique time-like direction. Further, in flat spacetime, Wick rotation can be physically interpreted in terms of causality and time-ordering, whereas there is no equivalent interpretation for curved spacetime. Both of these are needed in order to effectively formulate an EQG.

\subsection{Arrangement of the Thesis}

In this thesis there are two themes of study. We present a new analysis of the greybody factors for Schwarzschild black holes and review and clarify the possible approaches to a generalised Wick rotation. The work is divided into five main chapters. The first three are devoted to presenting the necessary physical concepts to understanding these problems. Chapters 2 and 3 review general relativity and the fundamentals of quantum field theory in flat spacetime. Chapter 4 presents the extension of QFT to curved spacetime and builds up to the derivation of Hawking radiation. We present two routes to this; the first is based on Hawking's original work, the second is based on Wick rotation. In the fifth chapter we develop a new formalism based on the product calculus to calculate the greybody factors and apply it to Schwarzschild black holes. It has the scope to be applied to a wide range of scattering problems not limited to general relativity. The material from this chapter has been incorporated into two papers. The first of which, in collaboration with Sebastian Schuster, Matt Visser, and Alexander Van-Brunt, has been published in Classical and Quantum Gravity [1. The second, in 
collaboration with Matt Visser, is in the submission process and available online on the arXiv [2]. Finally, in Chapter 6] we review the extension of Wick rotation, present the alternatives to a continuation $t \rightarrow-i t$, and suggest new directions for future work. 


\section{Chapter 2}

\section{Aspects of General Relativity}

In this chapter we introduce concepts from Einstein's general theory of relativity necessary for the latter material in this thesis. The first section introduces the basic manifold structure required and develops some results for hypersurfaces. The second section builds up to the Einstein equations then presents some properties of black holes. Finally the third section develops the Lagrangian formalism and shows how to derive the Einstein equations from the Einstein-Hilbert action. For fuller treatments of the aspects of general relativity presented here see the text books by Wald [3], and Misner, Thorne, and Wheeler [4]. In particular we follow Poisson's book [5].

\section{$2.1 \quad$ Preliminaries}

At its heart Einstein's general relativity represents the gravitational field as a metric (Christoffel) connection on a pseudo-Riemaninan manifold known as the spacetime and demands that free-fall correspond to geodesic motion in the manifold. This incorporates the equivalence of inertial and gravitational mass as a geometrical property at a fundamental level (See [3, 4] for more details). In this setting space and time are treated on an equal footing as the dimensions of the manifold and in particular can interchange dynamically. Standard general relativity takes place in $(3+1)$ dimensions; explicitly $x^{0}=c t$ is usually reserved for the time-like coordinate, while $x^{i}$, for $i \in\{1,2,3\}$, denote the three ordinary spatial coordinates. Hereafter we work in natural units such that the physical constants $c=\hbar=G=k_{B}=1$. A point in 
spacetime is sometimes referred to as an event.

\subsubsection{Distances and Differentiation}

The most important feature of a manifold in standard general relativity is the symmetric metric tensor, $g_{a b}$, which defines a canonical isomorphism between the tangent and co-tangent spaces. It is used to define the inner product between two vectors and hence measure distances. For example the infinitesimal distance between two points is given by,

$$
\mathrm{d} s^{2}=g_{a b} \mathrm{~d} x^{a} \mathrm{~d} x^{b}
$$

We use Einstein summation convention throughout, wherein, a repeated index indicates a sum over that index. The metric is also used to define the volume element in the manifold,

$$
\mathrm{d} V=\sqrt{|g|} \mathrm{d}^{4} x
$$

where $g$ is the determinant of the metric. The inverse metric $g^{a b}$ is defined as the inverse matrix of $g_{a b}$ treated as a $T_{0}^{2}$ tensor. Thus

$$
g_{a m} g^{m b}=\delta_{b}^{a}
$$

Here $\delta_{b}^{a}$ is the Kronecker delta.

A crucial feature of the metric is that it is not positive definite and has Lorentzian signature, with eigenvalues $(-,+,+,+)$. For example, the flat (Minkowski) spacetime metric can be written,

$$
\mathrm{d} s^{2}=-\mathrm{d} t^{2}+|\mathrm{d} \mathbf{x}|^{2} .
$$

This implies that distances can be any real number and encodes the causal structure of the spacetime. We say two events in Minkowski space $X_{1}, X_{2}$, are time-like separated, iff

$$
\Delta X=g_{a b}\left(X_{1}^{a}-X_{2}^{a}\right)\left(X_{1}^{b}-X_{2}^{b}\right)<0
$$

From eq. 2.4 and eq. 2.5 we have that the speed required to travel between 
$X_{1}$ and $X_{2}$ is less than 1 (i.e. the speed of light), and hence massive objects can travel a path between the two events. The separation is called light-like (or null) iff $\Delta X=0$, and the two events can be connected by a light ray. Iff $\Delta X>0$ the separation is space-like and the two events can never interact. These definitions also apply to any vector $V$ in the spacetime. Now, the set of all points

$$
I(X) \equiv\{Y: g([X-Y],[X-Y]) \leq 0\}
$$

defines all the events that can be causally related to $X$. Depending on the sign of $\Delta t$ they are future events affected by $X$ or past events which affect $X$.

These notions can be extended to a generic spacetime by defining two points on the manifold to be time-like separated iff there exists a curve between the two points whose tangent vector is everywhere time-like. The case of null and space-like separations follows immediately. A spacetime is called time orientable if non-space-like vectors can smoothly be assigned as future or past directed over the entire manifold. That is, there exists an everywhere time-like vector field $V$ defined on the entire manifold. We return to this in Chapter 6.

The metric also provides a natural way to differentiate on the manifold. Without going into details, a simple calculation shows that partial derivatives of anything other than scalars no longer behave as tensors. A covariant derivative of a tensor, which is a linear map from a $T_{s}^{r}$ tensor to a $T_{s+1}^{r}$ tensor, can be defined by,

$$
\begin{aligned}
\nabla_{a} X_{b_{1}, \ldots, b_{s}}^{a_{1}, \ldots, a_{r}}= & \partial_{a} X_{b_{1}, \ldots, b_{s}}^{a_{1}, \ldots, a_{r}}+\sum_{\alpha=1}^{r} \Gamma_{m a}^{a_{\alpha}} X_{b_{1}, \ldots, b_{s}}^{a_{1}, \ldots, m, \ldots, a_{r}} \\
& -\sum_{\sigma=1}^{s} \Gamma_{b_{\sigma} a}^{m} X_{b_{1}, \ldots, m, \ldots, b_{s}}^{a_{1}, \ldots, a_{r}}
\end{aligned}
$$

where,

$$
\Gamma_{b c}^{a}=\frac{1}{2} g^{a m}\left(\partial_{c} g_{m b}+\partial_{b} g_{m c}-\partial_{m} g_{b c}\right),
$$

is the Christoffel connection. It is not a tensor itself. However, the combination with the partial derivative is. Other connections may be defined, but 
eq. (2.8) is the unique connection compatible with the metric, i.e. $\nabla_{a} g_{b c}=0$. We will frequently use the notation $X_{, a}$ to indicate a partial derivative and the notation $X_{; a}$ to indicate the covariant derivative. Further we use the notation of square brackets $X_{[a|b| c]}$ to denote antisymmetrisation on the indices within the brackets. The index $b$ within || is ignored in this process. Symmetrisation is denoted with ordinary brackets.

We can also define the Lie derivative without reference to the metric. It is a generalisation of a directional derivative and defined along a vector $V$ by,

$$
\begin{aligned}
\mathcal{L}_{V} f=V^{a} \partial_{a} f: & \text { for a scalar } f, \\
\left\{\mathcal{L}_{V} T\right\}^{a}=[V, T]^{a}=V^{m} \partial_{m} T^{a}-T^{m} \partial_{m} V^{a}: & \text { for a vector } T, \\
\left\{\mathcal{L}_{V} U\right\}_{a}=V^{m} \partial_{m} U_{a}+U_{m} \partial_{a} V^{m} a: & \text { for a covector } U .
\end{aligned}
$$

It also obeys the Leibniz rule, $\mathcal{L}_{V}(X Y)=\mathcal{L}_{V}(X) Y+X \mathcal{L}_{V}(Y)$. Lie derivatives are important for representing symmetries of the manifold.

With this formalism the relevant physical equations from special relativity can be usually translated into a general relativistic context by the prescription $\partial \rightarrow \nabla$.

It was noted that free-fall is determined by geodesic motion in the spacetime. Now we explicitly define and present the geodesic equation. Most generally geodesics represent the straightest possible lines on a given manifold. These are determined by the choice of connection, since, the above statement is equivalent to demanding that the tangent vector to a curve $x^{a}(\lambda)$ in the manifold be parallel to itself when transported along the curve. That is,

$$
\frac{d x^{a}}{d \lambda} \nabla_{a}\left(\frac{d x^{b}}{d \lambda}\right)=\kappa(\lambda) \frac{d x^{b}}{d \lambda},
$$

for some scalar $\kappa(\lambda)$. With the change of parameter,

$$
\frac{d \bar{\lambda}}{d \lambda}=\exp \left(\int_{\lambda_{0}}^{\lambda} \kappa\left(\lambda^{\prime}\right) \mathrm{d} \lambda^{\prime}\right)
$$

the geodesic equation can be simplified to,

$$
\frac{d x^{a}}{d \lambda} \nabla_{a}\left(\frac{d x^{b}}{d \lambda}\right)=0 .
$$


The parameter $\bar{\lambda}$ is known as an affine parameter. Proper time is an example of an affine parameter.

Finally, we briefly mention Killing vectors before moving on. A Killing vector $K^{a}$ satisfies the equation,

$$
\left\{\mathcal{L}_{K} g\right\}_{a b}=\nabla_{a} K_{b}+\nabla_{b} K_{a}=0
$$

They are important because they represent symmetries in the metric. For example if the metric is independent of a particular coordinate, $x^{i}$, then vector $\delta_{i}^{a}$ is a Killing vector. Further each Killing vector represents a quantity which is conserved along geodesics. That is, suppose $\lambda$ is now an affine parameter, then,

$$
\frac{d}{d \lambda}\left(K_{a} \frac{d x^{a}}{d \lambda}\right)=0
$$

We now turn to hypersurfaces in a given spacetime and present some key features and a version of Stokes' theorem.

\subsubsection{Hypersurfaces and Stokes' Theorem}

In a four dimensional manifold we define a hypersurface, $\Sigma$, to be a three dimensional sub-manifold which is described by either a level-surface,

$$
\Phi\left(x^{a}\right)=0
$$

or by parametric equations

$$
x^{a}=x^{a}\left(y^{\alpha}\right) .
$$

Here $y^{\alpha}$ are the coordinates on the hypersurface, $\alpha \in\{1,2,3\}$. The hypersurface is called time-like, null, or space-like depending on whether the normal vector to it $\bar{n}^{a}$ is time-like, null, or space-like. If $\Sigma$ is time(space)-like the unit normal $n_{a}$ is given by

$$
n_{a}=\varepsilon \frac{\Phi_{, a}}{\left|g^{a b} \Phi_{, a} \Phi_{, b}\right|},
$$


where, $n_{a} n^{a}=\varepsilon=\mp 1$, refers to a time(space)-like hypersurface. This unit normal is not defined for a null surface as, $g^{a b} \Phi_{, a} \Phi_{, b}=0$. In this case let $k_{a}=-\Phi_{, a}$ be the normal where the sign ensures it is future directed. It should be noted that $k_{a}$ is also tangent to the hypersurface as $k_{a} k^{a}=0$ on $\Sigma$. From this, the gradient $\left(k^{a} k_{a}\right)_{; b}$ must be tangential to $k_{a}$. Thus one can show that $k_{a}$ automatically satisfies the general form of the geodesic equation $k^{a} \nabla_{a} k^{b}=\kappa k^{b}$ for some scalar $\kappa$. The null surfaces are hence generated by null geodesics parametrised by some (not necessarily affine) $\lambda$. Thus a change along a generator is described by $\mathrm{d} x^{a}=\lambda \mathrm{d} k^{a}$. Therefore we can describe these surfaces by the coordinates $y^{\alpha}=\left(\lambda, z^{A}\right), A \in\{2,3\}$. Null hypersurfaces will be important in the study of black hole horizons.

On the hypersurfaces we can define an induced metric using the tangent vectors to $\Sigma$,

$$
e_{\alpha}^{a}=\frac{\partial x^{a}}{\partial y^{\alpha}}
$$

Since these vectors are tangent to the hypersurface $e_{\alpha}^{a} n_{a}=0=e_{\alpha}^{a} k_{a}$. For line-elements on $\Sigma$,

$$
\mathrm{d} s_{\Sigma}^{2}=h_{\alpha \beta} \mathrm{d} y^{\alpha} \mathrm{d} y^{\beta}
$$

where,

$$
h_{\alpha \beta}=g_{a b} e_{\alpha}^{a} e_{\beta}^{b}
$$

This simplifies in the null case. Using the coordinates $y^{\alpha}=\left(\lambda, z^{A}\right)$, as then $e_{1}^{a}=\left(\partial x^{a} / \partial \lambda\right)_{\theta_{A}} \equiv k^{a}$. Thus $h_{11}=g_{a b} k^{a} k^{b}=0=g_{a b} k^{a} e_{A}^{b}=h_{1 A}$, and therefore

$$
\mathrm{d} s_{\Sigma}^{2}=\sigma_{A B} \mathrm{~d} z^{A} \mathrm{~d} z^{B}
$$

where

$$
\sigma_{A B}=g_{a b} e_{A}^{a} e_{B}^{a} \quad: \quad e_{A}^{a}=\left(\frac{\partial x^{a}}{\partial z^{A}}\right)_{\lambda} .
$$

Now in the case of time(space)-like hypersurfaces the inverse metric can be written

$$
g^{a b}=\varepsilon n^{a} n^{b}+h^{\alpha \beta} e_{\alpha}^{a} e_{\beta}^{b}
$$

where $h^{\alpha \beta}$ is the inverse of the induced metric. The right hand term defines a $4 \mathrm{D}$ version of the induced metric, $h^{a b} \equiv h^{\alpha \beta} e_{\alpha}^{a} e_{\beta}^{b}$, which can be used to project quantities onto the hypersurface. In the null case it is necessary to introduce an auxiliary null vector, $N^{a}$, satisfying $N_{a} k^{a}=-1, N_{a} e_{\alpha}^{a}=0$ on 
the surface. Then the inverse metric can be written

$$
g^{a b}=-k^{a} N^{b}-k^{b} N^{a}+\sigma^{A B} e_{A}^{a} e_{B}^{b},
$$

where $\sigma^{A B}$ is the inverse of $\sigma_{A B}$. Now for time(space)-like surfaces the surface element may be written

$$
\mathrm{d} \Sigma=\sqrt{|h|} \mathrm{d}^{3} y
$$

with $h \equiv \operatorname{det}\left[h_{\alpha \beta}\right]$. This can be extended to the outward directed surface element $n_{a} \mathrm{~d} \Sigma$, that is, in the direction of increasing $\Phi$. For the null case this can be written,

$$
\mathrm{d} \Sigma_{a}=-k_{a} \sqrt{\sigma} \mathrm{d} \lambda \mathrm{d}^{2} z
$$

where $\sigma \equiv \operatorname{det}\left[\sigma_{A B}\right]$. We are now in a position to present a version of Stokes' theorem without an excursion into differential forms.

Theorem 2.1. Consider a finite volume $\mathcal{V}$ of spacetime with closed boundary $\partial \mathcal{V}$ then for any vector $A^{a}$,

$$
\int_{\mathcal{V}} \nabla_{a} A^{a} \sqrt{|g|} \mathrm{d}^{4} x=\oint_{\partial \mathcal{V}} A^{a} \mathrm{~d} \Sigma_{a}
$$

Eq. 2.29 will be necessary for the Lagrangian Formulation of general relativity. (No proof will be given for this standard result).

It will be also necessary to introduce the notion of extrinsic curvature for the consistency of the action. For this, we now consider only time(space)-like hypersurfaces. The vectors $e_{\alpha}^{a}$ can be used to project quantities on to $\Sigma$. Consider the projection of the covariant derivative of a tangent vector to the surface, $e_{\alpha}^{a} \nabla_{a} A^{b}$. That is a vector such that,

$$
A^{a}=A^{\alpha} e_{\alpha}^{a}, \quad A^{a} n_{a}=0, \quad A_{\alpha}=A_{a} e_{\alpha}^{a} .
$$

The question remains whether the projected derivative has a normal compo- 
nent to the surface as well as a tangential component. Now

$$
\begin{aligned}
e_{\alpha}^{a} \nabla_{a} A^{b} & =g^{b}{ }_{c} A_{; a}^{c} e_{\alpha}^{a} \\
& =\left(\varepsilon n^{b} n_{c}+h^{\mu \beta} e_{\beta}^{b} e_{\mu c}\right) A_{; a}^{c} e_{\alpha}^{a} \\
& =\varepsilon\left(n_{c} A_{; a}^{c} e_{\alpha}^{a}\right) n^{b}+h^{\mu \beta}\left(A_{c ; a} e_{\mu}^{c} e_{\alpha}^{a}\right) e_{\beta}^{b} \\
& =-\varepsilon A^{\gamma}\left(n_{c ; a} e_{\alpha}^{a} e_{\gamma}^{c}\right) n^{b}+h^{\mu \beta}\left(A_{c ; a} e_{\mu}^{c} e_{\alpha}^{a}\right) e_{\beta}^{b} .
\end{aligned}
$$

In the last line we have used the orthogonality of $n_{a} A^{a}=0$. The right hand term can be seen to be tangential to the surface, however the left hand term is normal to it. Defining the extrinsic curvature

$$
K_{\alpha \beta}=n_{a ; b} e_{\alpha}^{a} e_{\beta}^{b}
$$

the orthogonal component vanishes when the extrinsic curvature vanishes. Thus the extrinsic curvature contains the information on how the surface is embedded in the spacetime and how its normal vector varies.

\subsection{Curvature and Field Equations}

Up to this point we have assumed that the spacetime metric was known and it was used to describe the physics in a particular geometry. We now turn to the equations describing the formation and evolution of these spacetimes in vacua and the presence of matter.

The information of the curvature of a spacetime is contained in the Riemann tensor, which represents how much covariant derivatives fail to commute. That is,

$$
\left[\nabla_{a}, \nabla_{b}\right] X^{c}=R_{d a b}^{c} X^{d}
$$

for a symmetric connection. Here the Riemann tensor is given by

$$
R_{b c d}^{a}=-2 \Gamma_{b[c, d]}^{a}+2 \Gamma_{m[c}^{a} \Gamma_{|b| d]}^{m}
$$

The Ricci tensor is given by contracting on the first and third index

$$
R_{a b}=R_{a m b}^{m}
$$


This has, in general, only ten independent components in four dimensions due to the symmetries of the metric and Riemann tensor.

Now the Einstein equations in vacuum, state that the Ricci curvature is identically zero

$$
R_{a b}=0
$$

Note that generically this is a set of non-linear coupled second order differential equations in the components of the metric. Further, the vanishing of the Ricci tensor does not imply that the geometry is Riemann flat. Some of the most important spacetimes are derived from the vacuum equations.

If there is matter present with an associated stress-energy tensor, $T_{a b}$ then the field equations become

$$
G_{a b}=8 \pi T_{a b},
$$

where the Einstein tensor is defined,

$$
G_{a b} \equiv R_{a b}-\frac{1}{2} R g_{a b}
$$

with the Ricci scalar $R \equiv g^{a b} R_{a b}$. Succinctly these equations state, as Wheeler put it [6], "spacetime tells matter how to move; matter tells spacetime how to curve". The Einstein tensor is chosen rather than, for example, the Ricci tensor as it satisfies the Bianchi identity $\nabla_{a} G^{b c}=0$, thus the stress-energy tensor is automatically covariantly conserved,

$$
\nabla_{a} T^{b c}=0
$$

\subsubsection{Black Holes and Horizons}

One of the simplest solutions to the vacuum field equations was found by Karl Schwarzschild, in 1916, for the case of a static spherically symmetric body. The metric in standard coordinates is given by,

$$
\mathrm{d} s^{2}=-\left(1-\frac{2 M}{r}\right) \mathrm{d} t^{2}+\left(1-\frac{2 M}{r}\right)^{-1} \mathrm{~d} r^{2}+r^{2}\left(\mathrm{~d} \theta^{2}+\sin ^{2} \theta \mathrm{d} \varphi^{2}\right)
$$

This has a coordinate singularity at $r=2 M$, and a curvature singularity at $r=0$. Now the Birkhoff theorem [5] shows that it is the unique solution for 
spacetime outside a spherically symmetric massive body of mass $M$. By the coordinate transform,

$$
\begin{aligned}
& U=-4 M e^{-u / 4 M} \\
& V=4 M e^{v / 4 M}
\end{aligned}
$$

where, $u=t-r_{*}, v=t+r_{*}$, and $r_{*}=r+2 M \ln (r / 2 M-1)$, the metric can be put into Kruskal-Szekeres form,

$$
d s^{2}=-\frac{2 M}{r} e^{-r / 2 M} \mathrm{~d} U \mathrm{~d} V+r^{2}\left(\mathrm{~d} \theta^{2}+\sin ^{2} \theta \mathrm{d} \varphi^{2}\right)
$$

which is manifestly finite at $r=2 M$. In these coordinates, ingoing and outgoing light rays correspond to curves, $V=$ const, and, $U=$ const, respectively. The spacetime can be visually represented in a conformal Penrose-Carter diagram shown below in Fig. 2.1.

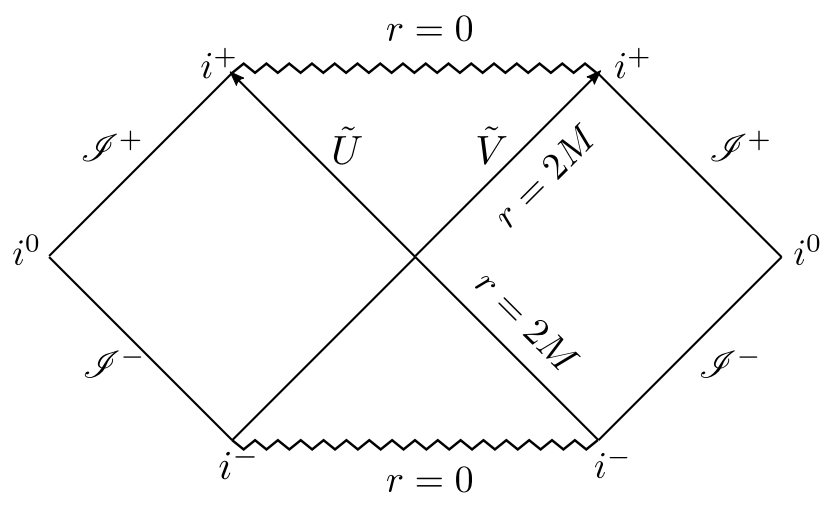

Figure 2.1: The conformal Penrose-Carter diagram for a Schwarzschild black hole.

Light rays are represented straight lines at $45^{\circ}$ on the diagram with outgoing rays having positive gradient. It can be seen that no outgoing ray with $r<2 M$ (i.e. $U<0$ ) can escape the singularity at $r=0$. This means that nothing can escape a body whose surface is less than the Schwarzschild radius $r_{s}=2 M$ once it crosses this boundary. This is what defines a black hole. The points $\mathscr{I}^{+(-)}$are called future (past) null infinity and represent all possible future (past) end points of outgoing (ingoing) null geodesics. $i^{+(-)}$ are the future and past times $(t \rightarrow \pm \infty$ for finite $r)$ and $i^{0}$ is spatial infinity $(r \rightarrow \infty$ for finite $t)$. 
The surface at $r=2 M$ is called the event horizon. It is the point where the time-like killing $t^{a}=\partial x^{a} / \partial t$ vector becomes null and hence it is a null-surface. On this surface the null generator, as before, must satisfy the geodesic equation,

$$
t^{a} \nabla_{a} t^{b}=\kappa t^{a}
$$

$\kappa$ is called the surface gravity and represents the force at spatial infinity required to hold a test particle stationary on the horizon. It can be shown to be given by

$$
\kappa^{2}=-\frac{1}{2} \nabla^{a} t^{b} \nabla_{a} t_{b}
$$

There are other solutions describing different kinds of black holes e.g. the Kerr solution for rotating black holes. These all present similar features and have an event horizon described by a null surface from inside which light rays cannot escape. Further they have a surface gravity given by eq. (2.45).

\subsection{Lagrangian Formulation}

One of the most beautiful aspects of general relativity is that it can be put into the language of least action principle with a strikingly simple Lagrangian. We shall see later that this is suggestive of a possible quantisation of the gravitational field via the Feynman path integral method. We now briefly review the least action principle and Euler-Lagrange equations.

\subsubsection{Euler-Lagrange Review}

A classical field theory can be described by a given Lagrangian, $\mathscr{L}\left(q, q_{, a}\right)$ which is a scalar function of the field, $q\left(x^{a}\right)$ (later we will generalise this to tensor fields), and its spatial derivatives defined on a volume of spacetime $\mathcal{V}$ with closed boundary $\partial \mathcal{V}$. One writes the action functional which integrates the Lagrangian over the volume of spacetime for a particular field configuration,

$$
S[q]=\int_{\mathcal{V}} \mathscr{L}\left(q, q_{, a}\right) \sqrt{-g} \mathrm{~d}^{4} x
$$

The equations of motion are then found by finding the extrema of the action, where $\delta S=0$, under variation of the field, $q+\delta q$. This is subject to 
appropriate boundary conditions such that the variation $\delta q$ vanish on the boundary

$$
\left.\delta q\right|_{\partial \mathcal{V}}=0
$$

Now

$$
\begin{aligned}
\delta S[q] & =\int_{\mathcal{V}} \delta\left[\mathscr{L}\left(q, q_{, a}\right) \sqrt{-g}\right] \mathrm{d}^{4} x \\
& =\int_{\mathcal{V}}\left(\frac{\partial \mathscr{L}}{\partial q} \delta q+\frac{\partial \mathscr{L}}{\partial q_{, a}}(\delta q)_{, a}\right) \sqrt{-g} \mathrm{~d}^{4} x \\
& =\int_{\mathcal{V}}\left(\frac{\partial \mathscr{L}}{\partial q} \delta q+\left[\frac{\partial \mathscr{L}}{\partial q_{, a}} \delta q\right]_{; a}-\left[\frac{\partial \mathscr{L}}{\partial q_{, a}}\right]_{; a} \delta q\right) \sqrt{-g} \mathrm{~d}^{4} x \\
& =\int_{\mathcal{V}}\left(\frac{\partial \mathscr{L}}{\partial q}-\left[\frac{\partial \mathscr{L}}{\partial q_{, a}}\right]_{; a}\right) \delta q \sqrt{-g} \mathrm{~d}^{4} x+\oint_{\partial \mathcal{V}} \frac{\partial \mathscr{L}}{\partial q_{, a}} \delta q \mathrm{~d} \Sigma_{a} .
\end{aligned}
$$

In the last line we have used Stokes' theorem eq. 2.29. But by the boundary condition eq. (2.47) the right most term vanishes and hence the equations of motion are,

$$
\frac{\partial \mathscr{L}}{\partial q}-\left[\frac{\partial \mathscr{L}}{\partial q_{, a}}\right]_{; a}=0 .
$$

With this background in mind, we now show how to translate general relativity into this language.

\subsubsection{Einstein-Hilbert Action}

The action for general relativity is a functional of the metric tensor $g_{a b}$. It contains a curvature term we denote $S_{H}[g]$, a boundary term, $S_{B}[g]$, and a constant, $S_{0}$. The full action, for a volume $\mathcal{V}$ of spacetime with closed non-null boundary $\partial \mathcal{V}$ and outward pointing normal vector $n_{a}$, is written,

$$
S_{G}[g]=S_{H}[g]+S_{B}[g]-S_{0},
$$


where

$$
\begin{aligned}
S_{H}[g, \phi] & =\frac{1}{16 \pi} \int_{\mathcal{V}} R \sqrt{-g} \mathrm{~d}^{4} x \\
S_{B}[g] & =\frac{1}{8 \pi} \oint_{\partial \mathcal{V}} \varepsilon K \sqrt{|h|} \mathrm{d}^{3} y \\
S_{0} & =\frac{1}{8 \pi} \oint_{\partial \mathcal{V}} \varepsilon K_{0} \sqrt{|h|} \mathrm{d}^{3} y
\end{aligned}
$$

Here $R$ is the Ricci scalar, $K=\nabla_{a} n^{a}$ is the trace of the extrinsic curvature of the boundary $\partial \mathcal{V}$, and $\varepsilon= \pm 1$ in the regions where $\partial \mathcal{V}$ is time(space)-like. $S_{0}$ will be explained latter.

The Hilbert term is the simplest action which is invariant under diffeomorphisms (i.e. a scalar) and contains derivatives of the metric. However since these derivatives are second order and we do not fix the variation of derivatives of the metric on the boundary, the boundary terms are necessary for the consistency of the action when the volume is non-compact. We will now show that under variation of the metric $\delta g_{a b}$ subject to the boundary conditions,

$$
\left.\delta g_{a b}\right|_{\partial \mathcal{V}}=0
$$

the extrema of the action leads to the Einstein field equations. Note this condition also implies the variation of the induced metric $h^{a b}$ is zero on the boundary. To aid us in the computation we note the following results,

$$
\begin{aligned}
\delta g_{a b} & =-g_{a m} g_{b n} \delta g^{m n} \\
\delta \sqrt{-g} & =-\frac{1}{2} \sqrt{-g} g_{a b} \delta g^{a b} \\
\delta R_{a b} & =\nabla_{m} \delta \Gamma_{b a}^{m}-\nabla_{b} \delta \Gamma_{m a}^{m}, \\
\left.\delta \Gamma_{b c}^{a}\right|_{\partial \mathcal{V}} & =\frac{1}{2} g^{a m}\left(\delta g_{m b, c}+\delta g_{m c, c}-\delta g_{b c, m}\right) .
\end{aligned}
$$

In the last identity the fact that the variation of the metric is zero on the boundary is used to convert the usual covariant derivatives of $\delta g_{a b}$ into partial derivatives. 
Varying the Hilbert term and using the above results leads to,

$$
\begin{aligned}
16 \pi \delta S_{H} & =\int_{\mathcal{V}}\left(R_{a b} \delta g^{a b}-\frac{1}{2} R g_{a b} \delta g^{a b}+\nabla_{m}\left[g^{a b} \delta \Gamma_{a b}^{m}-g^{m n} \delta \Gamma_{a n}^{a}\right]\right) \sqrt{-g} \mathrm{~d}^{4} x \\
& =\int_{\mathcal{V}} G_{a b} \delta g^{a b} \sqrt{-g} \mathrm{~d}^{4} x+\varepsilon \oint_{\partial \mathcal{V}}\left[g^{a b} \delta \Gamma_{a b}^{c}-g^{c d} \delta \Gamma_{a d}^{a}\right] n_{c} \sqrt{|h|} \mathrm{d}^{3} y,
\end{aligned}
$$

where in the second line we have used Stokes' theorem eq. 2.29) and $n_{c}$ is the unit normal to the surface. Now using the last of the identities we have $g^{a b} \delta \Gamma_{a b}^{c}-g^{c d} \delta \Gamma_{a d}^{a}=g^{c m} g^{a b}\left(\delta g_{m b, a}-\delta g_{a b, m}\right)$, and the right most term becomes,

$$
\begin{aligned}
& \varepsilon \oint_{\partial \mathcal{V}}\left[g^{a b}\left(\delta g_{c b, a}-\delta g_{a b, c}\right)\right] n^{c} \sqrt{|h|} \mathrm{d}^{3} y \\
& =\varepsilon \oint_{\partial \mathcal{V}}\left(\varepsilon n^{a} n^{b}+h^{a b}\right)\left(\delta g_{c b, a}-\delta g_{a b, c}\right) n^{c} \sqrt{|h|} \mathrm{d}^{3} y \\
& =\varepsilon \oint_{\partial \mathcal{V}} h^{a b}\left(\delta g_{c b, a}-\delta g_{a b, c}\right) n^{c} \sqrt{|h|} \mathrm{d}^{3} y .
\end{aligned}
$$

In the third line we have used the antisymmetry to get rid of the term multiplied by $n^{a} n^{c}$. Further, since the variation of the metric is zero on the boundary, its tangential derivative must also vanish. This implies $\delta g_{a b, c} h^{c d}=$ 0 and we are left with,

$$
-\varepsilon \oint_{\partial \mathcal{V}} h^{a b} \delta g_{a b, c} n^{c} \sqrt{|h|} \mathrm{d}^{3} y
$$

This term is exactly equal in magnitude and opposite in sign to the variation of the boundary term $\delta S_{B}$. That is, using the identity

$$
K=h^{a b} n_{a ; b}=h^{a b}\left(n_{a, b}-\Gamma_{a b}^{c} n_{c}\right)
$$

we have

$$
\begin{aligned}
16 \pi \delta S_{B}[g] & =2 \varepsilon \oint_{\partial \mathcal{V}} \delta K \sqrt{|h|} \mathrm{d}^{3} y \\
& =-2 \varepsilon \oint_{\partial \mathcal{V}} h^{a b} \delta \Gamma_{a b}^{c} n_{c} \sqrt{|h|} \mathrm{d}^{3} y \\
& =\varepsilon \oint_{\partial \mathcal{V}} h^{a b} \delta g_{a b, c} n^{c} \sqrt{|h|} \mathrm{d}^{3} y
\end{aligned}
$$


In the third line we have used the fact that the tangential derivatives of the variation of the metric must vanish on the boundary.

Since $S_{0}$ depends only on the induced metric, through $\sqrt{h}$, its variation vanishes on the boundary. Thus now including a matter action of the form, $S_{m}=\int_{\mathcal{V}} \mathscr{L}_{m} \sqrt{-g} \mathrm{~d}^{4} x$, we are left with

$$
\delta\left[S_{G}+S_{m}\right]=\frac{1}{16 \pi} \int_{\mathcal{V}}\left(G_{a b}+\frac{\delta S_{m}}{\delta g^{a b}}\right) \delta g^{a b} \sqrt{-g} \mathrm{~d}^{4} x
$$

Hence defining the stress energy tensor, $T_{a b} \equiv-1 / 2 \delta S_{m} / \delta g_{a b}$, the extrema of the action $\delta S=0$ implies the Einstein equations,

$$
G_{a b}=8 \pi T_{a b}
$$

Finally the purpose of the non-dynamical $S_{0}$ term is to ensure the action remains finite. The value of the action for a solution to the vacuum equations (where $R=0$ ) is,

$$
S_{G}=S_{B}-S_{0}=\frac{1}{8 \pi} \oint_{\partial \mathcal{V}} \varepsilon\left(K-K_{0}\right) \sqrt{|h|} \mathrm{d}^{3} y
$$

In the limit of spatial infinity this term can be shown to be finite when $K_{0}$ is interpreted as being the value of the trace of the extrinsic curvature of the boundary $\partial \mathcal{V}$ when embedded in flat space. 


\section{Chapter 3}

\section{Aspects of Quantum Field Theory in Flat Spacetime}

In this chapter we introduce the basics of quantum field theory (QFT) in flat spacetime necessary for understanding the Hawking Effect in curved spacetime. We also highlight the usefulness of Wick rotation and Euclidean methods, and the correspondence with statistical mechanics. The are many text books on quantum field theory, in particular, we refer the reader to the books by Birrell and Davies [7] and Peskin and Schroeder [8] for the material presented here.

\subsection{Preliminaries and Canonical Quantisation}

There are two standard ways to quantising a field theory. In this section we review the basic concepts and present the canonical method in terms of creation and annihilation operators. We show results here for a scalar field and eschew the complications of higher spin.

\subsubsection{Free Scalar}

A free scalar field, $\phi\left(x^{a}\right)$, has the Lagrangian density,

$$
\mathscr{L}=-\frac{1}{2}\left(\partial^{a} \phi \partial_{a} \phi+m^{2} \phi^{2}\right)
$$


in flat space with metric $\eta_{a b}=\operatorname{diag}(-1,+1,+1,+1)$. The canonical conjugate momentum of the field is defined by

$$
\pi\left(x^{a}\right)=\frac{\partial \mathscr{L}}{\partial\left(\partial_{t} \phi\right)}=\partial_{t} \phi\left(x^{a}\right)
$$

This allows the Hamiltonian density to be constructed via the Legendre transform,

$$
\begin{aligned}
\mathscr{H} & =\pi(t, \mathbf{x}) \partial_{t} \phi(t, \mathbf{x})-\mathscr{L} \\
& =\frac{1}{2}\left[\left(\partial_{t} \phi\right)^{2}+(\nabla \phi)^{2}+m^{2} \phi^{2}\right] .
\end{aligned}
$$

Variation of the action, c.f. Chapter 2, leads to the Klein-Gordon equation,

$$
\left(-\square+m^{2}\right) \phi\left(x^{a}\right)=\left(\partial_{t}^{2}-\nabla^{2}+m^{2}\right) \phi\left(x^{a}\right)=0
$$

This has plane wave solutions,

$$
u_{\mathbf{k}}=\frac{1}{\sqrt{2 \omega}} e^{i \mathbf{k} \cdot \mathbf{x}-i \omega t}
$$

where, $\omega=\left(|\mathbf{k}|^{2}+m^{2}\right)^{1 / 2}$. These are called positive frequency modes with respect to $t$,

$$
\frac{\partial}{\partial t} u_{\mathbf{k}}(t, \mathbf{x})=-i \omega u_{\mathbf{k}}(t, \mathbf{x})
$$

for $\omega>0$.

One can define the inner product,

$$
\left(\phi_{1}, \phi_{2}\right)=-i \int \mathrm{d}^{3} x\left(\phi_{1}(x) \partial_{t} \phi_{2}^{*}(x)-\partial_{t}\left[\phi_{1}(x)\right] \phi_{2}^{*}(x)\right) \text {. }
$$

It can be seen that the positive frequency modes eq. (3.5) form a complete orthogonal basis and so the field can be expanded,

$$
\phi(t, \mathbf{x})=\sum_{\mathbf{k}}\left(a_{\mathbf{k}} u_{k}(t, \mathbf{x})+a_{\mathbf{k}}^{\dagger} u_{k}^{*}(t, \mathbf{x})\right)
$$

We shall often ignore the subtleties in switching between discrete and continuous expansions, in general the prescription $\sum_{\mathbf{k}} \rightarrow \int \frac{\mathrm{d}^{n} k}{(2 \pi)^{n}}$ can be employed. 


\subsubsection{Quantisation}

The canonical way to quantise a system is to promote the field to an operator in a Hilbert space, $\phi \rightarrow \hat{\phi}$, and impose the equal time commutation relations,

$$
\begin{aligned}
& {\left[\hat{\phi}(t, \mathbf{x}), \hat{\phi}\left(t, \mathbf{x}^{\prime}\right)\right]=0,} \\
& {\left[\hat{\pi}(t, \mathbf{x}), \hat{\pi}\left(t, \mathbf{x}^{\prime}\right)\right]=0,} \\
& {\left[\hat{\phi}(t, \mathbf{x}), \hat{\pi}\left(t, \mathbf{x}^{\prime}\right)\right]=i \delta^{(3)}\left(\mathbf{x}-\mathbf{x}^{\prime}\right)}
\end{aligned}
$$

where $\hat{\pi}$ is the canonical momentum operator, c.f eq. 3.2 and $\delta^{(3)}(x)$ is the three dimensional Dirac-delta function. Working in the Heisenberg picture, where the time evolution of an operator is given by $\left.\hat{\phi}(t, \mathbf{x})=e^{i \hat{H} t} \phi \hat{(\mathbf{x}}\right) e^{-i \hat{H} t}$, one can show that the operator $\hat{\phi}$ obeys the classical equation of motion eq. (3.4). Using the expansion of the field, eq. (3.8), the commutation relations are equivalent to imposing,

$$
\begin{aligned}
& {\left[\hat{a}_{\mathbf{k}}, \hat{a}_{\mathbf{k}^{\prime}}\right]=0,} \\
& {\left[\hat{a}_{\mathbf{k}}^{\dagger}, \hat{a}_{\mathbf{k}^{\prime}}^{\dagger}\right]=0,} \\
& {\left[\hat{a}_{\mathbf{k}}, \hat{a}_{\mathbf{k}^{\prime}}^{\dagger}\right]=\delta_{\mathbf{k k}^{\prime}} .}
\end{aligned}
$$

The operators $\hat{a}_{\mathbf{k}}^{(\dagger)}$ are called annihilation (creation) operators reminiscent of the quantum harmonic oscillator. In the Fock-Space representation of the Hilbert space the basis ket vectors, |\rangle , are built from the state, $|0\rangle$, called the quantum vacuum state. This vacuum state is Lorentz invariant and contains no particles; for all $\hat{a}_{\mathbf{k}}$,

$$
\hat{a}_{\mathbf{k}}|0\rangle=0
$$

Furthermore the operator $\hat{a}_{\mathbf{k}}^{\dagger}$ creates a particle in the state $\mathbf{k}$,

$$
\left|1_{\mathbf{k}}\right\rangle=\hat{a}_{\mathbf{k}}^{\dagger}|0\rangle
$$

Many particle states are then built by repeated application of creation operators to the vacuum state,

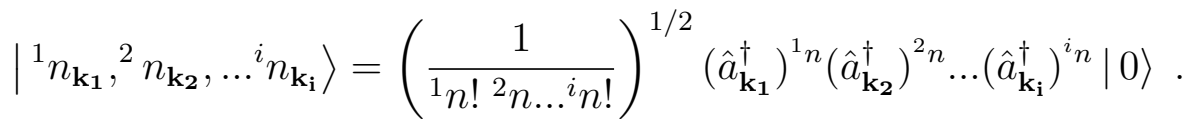


This state contains ${ }^{j} n$ particles in the mode $\mathbf{k}_{\mathbf{j}}$ and the pre-factor normalises the state. Application the of creation (annihilation) operator to a state $n_{\mathbf{k}}$ creates (destroys) a particle in this state, that is,

$$
\begin{aligned}
& \hat{a}_{\mathbf{k}}^{\dagger}\left|n_{\mathbf{k}}\right\rangle=\sqrt{n+1}\left|(n+1)_{\mathbf{k}}\right\rangle \\
& \hat{a}_{\mathbf{k}}\left|n_{\mathbf{k}}\right\rangle=\sqrt{n}\left|(n-1)_{\mathbf{k}}\right\rangle
\end{aligned}
$$

We can define the number operator,

$$
\hat{N}_{\mathbf{k}}=\hat{a}_{\mathbf{k}}^{\dagger} \hat{a}_{\mathbf{k}}
$$

which measures the number of particles in state $\mathbf{k}$, that is,

$$
\begin{gathered}
\left\langle 0\left|\hat{N}_{\mathbf{k}}\right| 0\right\rangle=0 \\
\left\langle{ }^{1} n_{\mathbf{k}_{\mathbf{1}}},{ }^{2} n_{\mathbf{k}_{\mathbf{2}}}, \ldots{ }^{i} n_{\mathbf{k}_{\mathbf{i}}}\left|\hat{N}_{\mathbf{k}_{\mathbf{j}}}\right|{ }^{1} n_{\mathbf{k}_{\mathbf{1}}},{ }^{2} n_{\mathbf{k}_{\mathbf{2}}}, \ldots{ }^{i} n_{\mathbf{k}_{\mathbf{i}}}\right\rangle={ }^{j} n .
\end{gathered}
$$

Using this the Hamiltonian operator c.f. eq. (3.3) can be written,

$$
\begin{aligned}
\hat{H} & =\sum_{\mathbf{k}}\left(\hat{a}_{\mathbf{k}}^{\dagger} \hat{a}_{\mathbf{k}}+\frac{1}{2}\right) \omega \\
& =\sum_{\mathbf{k}}\left(\hat{N}_{\mathbf{k}}+\frac{1}{2}\right) \omega .
\end{aligned}
$$

The sum $\sum_{\mathbf{k}} \frac{1}{2} \omega$ represents the vacuum energy.

So far we have presented the formalism of a many particle free scalar field theory. We now turn to the question of the probability for a particle to propagate from one point to another.

\subsubsection{Green Functions and Propagators}

The Feynman propagator is of great importance as it represents the amplitude (or probability) of propagation from $x^{a}$ to $y^{b}$. It is given as the expectation value of time ordered product of the fields, $\hat{\phi}$, at $x^{a}$ and $y^{b}$. 
That is,

$$
\begin{aligned}
D_{\mathrm{F}}(x-y) & =\left\langle 0\left|T\left\{\hat{\phi}\left(x^{a}\right) \phi\left(\hat{y^{a}}\right)\right\}\right| 0\right\rangle \\
& =\Theta\left(x^{0}-y^{0}\right)\left\langle 0\left|\hat{\phi}\left(x^{a}\right) \hat{\phi}\left(y^{b}\right)\right| 0\right\rangle+\Theta\left(y^{0}-x^{0}\right)\left\langle 0\left|\hat{\phi}\left(y^{b}\right) \hat{\phi}\left(x^{a}\right)\right| 0\right\rangle,
\end{aligned}
$$

where $T$ is the time ordering operation, and $\Theta(x)$ is the Heaviside step function. Using the commutation relations and the properties of $\Theta$ it can be shown that the propagator is a Green function of the Klein-Gordon equation. That is,

$$
\left(-\square+m^{2}\right) D_{F}\left(x^{a}-y^{b}\right)=-i \delta^{(4)}\left(x^{a}-y^{a}\right) .
$$

Here $\delta^{(4)}(x)$ is the Dirac-delta function in $4 \mathrm{D}$. An explicit expression for the propagator is given by,

$$
D_{\mathrm{F}}(x-y)=\int \frac{\mathrm{d}^{4} p}{(2 \pi)^{4}} \frac{-i}{p^{2}+m^{2}-i \epsilon} e^{i p \cdot(x-y)}
$$

where we have used the notation $p^{a}=(\omega, \mathbf{k}), \mathrm{d}^{4} p=\mathrm{d} \omega \mathrm{d}^{3} k$, so that $p^{2}=$ $-\omega^{2}+k^{2}$. The $i \epsilon$ is introduced to aid convergence; it is understood that the final result is obtained in the limit $\epsilon \rightarrow 0$. Often the Fourier transform of the propagator is a more useful representation, that is,

$$
D_{\mathrm{F}}(p)=\frac{-i}{p^{2}+m^{2}-i \epsilon} .
$$

At this point we have laid the background for a free scalar field. However the real complications of a quantum field theory come from the interactions between the particles of the field. Writing the interacting Hamiltonian as the sum, $H=H_{0}+V_{I}$, where $H_{0}$ is the free Hamiltonian and $V_{I}$ represents the interactions, one seeks to express the evolution of the interacting fields in terms of expressions of free field. That is, one seeks to find the propagator

$$
\langle\Omega|T\{\hat{\phi}(x) \hat{\phi}(y)\}| \Omega\rangle
$$

where $|\Omega\rangle$ represents the ground state of the interacting theory and $\hat{\phi}$ is governed by the full Hamiltonian. It turns out that there is a perturbative 
expansion for this in the free propagator eq. 3.27 whose terms can be formulated by Feynman diagrams. Using this one can then find the scattering amplitudes of various experimental configurations. Working with this perturbative expansion will not concern us in this thesis. Moreover, the formalism in this section can be generalised to other fields of higher spin accompanied by varying levels of complication, again that exercise is not the focus of this work.

\subsection{Path Integral Formulation}

The quantisation of a field can also be approached from the path integral representation of quantum mechanics. One advantage of this approach is that it is a priori Lorentz invariant for a Lorentz invariant Lagrangian and hence can be translated to a fully general relativistic setting more easily. In this formalism the quantum mechanical amplitude to propagate from $x^{a}$ to $y^{b}$ is the sum over all paths from $x^{a}$ to $y^{b}$ with a phase factor given by the value of the classical action along that path. Each path is equi-probable but carries a different phase. This becomes, for a field $\phi$ with source $J$, the functional integral representing the transition between initial and final vacua $\mid 0$, in $\rangle, \mid 0$, out $\rangle$,

$$
Z[J]=\langle\text { out, } 0| 0, \text { in }\rangle=\int D[\phi] \exp \left(i S[\phi]+i \int \mathrm{d}^{4} x J(x) \phi(x)\right),
$$

where $D[\phi]$ is the appropriate measure of the space of functions $\phi$ and $S[\phi]$ is the classical action. See [9] for a detailed approach to this construction. The connected Feynman diagrams for the perturbation theory of an interacting field, as described above, are given by functional differentiation,

$$
\left\langle 0\left|T\left\{\phi\left(x_{1}\right) \ldots \phi\left(x_{j}\right)\right\}\right| 0\right\rangle=\left.(-i)^{j} \frac{\delta^{j} \ln Z}{\delta J\left(x_{1}\right) . . \delta J\left(x_{j}\right)}\right|_{J=0} .
$$

These are effectively terms in a Taylor series type approximation to eq. (3.30). In special cases, for example, a free field this can be solved exactly. Defining the operator

$$
K_{x y}=\left(-\square_{x}+m^{2}-i \epsilon\right) \delta^{4}(x-y)
$$


which has inverse $K_{x y}^{-1}=i D_{\mathrm{F}}(x-y)$ and using techniques of Gaussian integrals for matrices eq. 3.30 becomes

$$
Z[J] \propto \operatorname{det}\left[K^{1 / 2}\right]^{-1} \exp \left(-\frac{1}{2} \int \mathrm{d}^{4} x \mathrm{~d}^{4} y J(x) D_{\mathrm{F}}(x-y) J(y)\right) .
$$

Thus it follows from eq. (3.31) that

$$
\langle 0|T\{\phi(x) \phi(y)\}| 0\rangle=-\left.\frac{\delta^{2} \ln Z}{\delta J(x) \delta J(y)}\right|_{J=0}=D_{\mathrm{F}}(x-y),
$$

which agrees with the previous expression.

\subsection{Wick Rotation and Euclidean Methods}

In many cases the calculations of QFT in Minkowski space can be made easier by employing a Wick rotation sending time to imaginary time, $t \rightarrow \tau=-i t$. In this section we present some examples of such calculations as motivation for Chapter 6 of this thesis, in which, we consider how to generalise this to a procedure in curved spacetime.

\subsubsection{Euclidean Propagators}

The Feynman propagator, e.q (3.27), has poles at $\omega^{2}=\mathbf{k}^{2}+m^{2}-i \epsilon=0$. That is, using the infinitesimal property of $\epsilon$, for $\omega= \pm\left(\omega_{\mathbf{k}}-i \epsilon\right)$. One can perform the integral over $p^{0}$ by an excursion into the field of complex contour integrals and the residue theorem. When $x^{0}>y^{0}$ the contour can be closed by a semi-circle in the lower half plane picking up the pole at $\omega_{\mathbf{k}}-i \epsilon$. When $y^{0}>x^{0}$ one can close the contour with a semicircle in the upper half plane, which picks up the pole $-\omega_{\mathbf{k}}+i \epsilon$ and gives the same propagator but with $x$ and $y$ reversed, that is, $D_{\mathrm{F}}(y-x)$. Thus the $i \epsilon$ prescription, which aids convergence, also encodes the information on the time ordering.

It can be seen that the original Feynman prescription for evaluating the contour is equivalent to Wick rotating $t \rightarrow \tau=-i t$ and $p^{0} \rightarrow \kappa=-i p^{0}$ which rotates the contour $90^{\circ}$ anticlockwise and picks up the same pole. This is shown below in Fig. 3.1. 


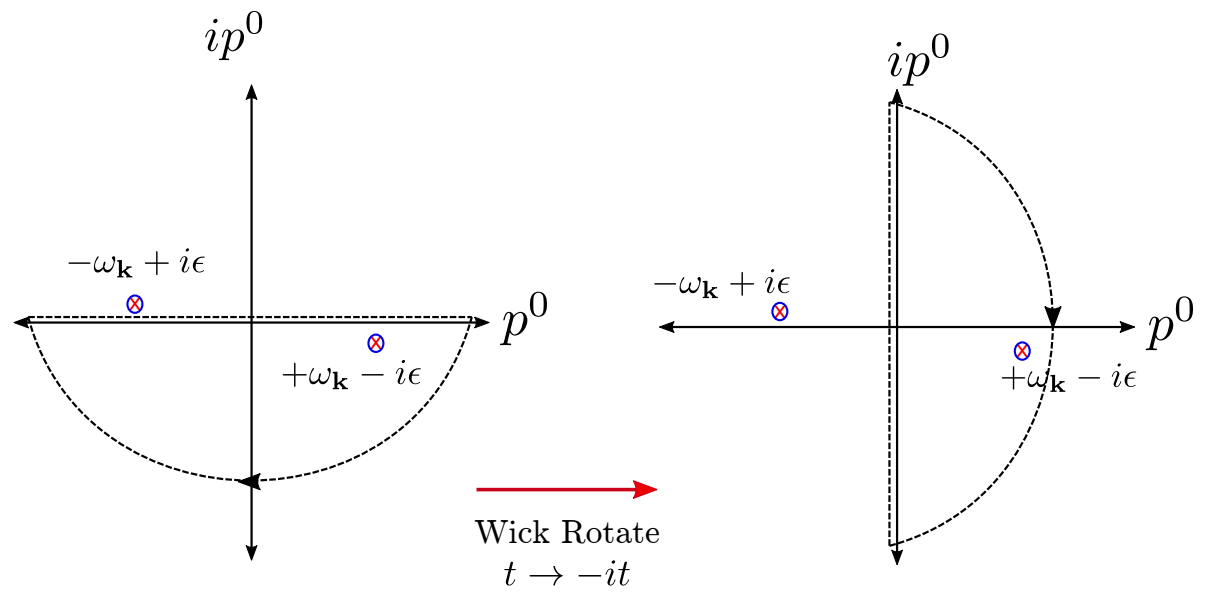

Figure 3.1: The contour integral of the time coordinate in the Feynman propagator is equivalent the Wick rotated contour along the imaginary time axis.

Doing this leads the relation between the propagators,

$$
D_{\mathrm{F}}\left(t, \mathbf{x} ; t^{\prime}, \mathbf{x}^{\prime}\right)=-i D_{\mathrm{E}}\left(i \tau, \mathbf{x} ; i \tau^{\prime}, \mathbf{x}^{\prime}\right)
$$

where $D_{\mathrm{E}}(x)$ is the Wick rotated (Euclidean) propagator. Further it can be seen in Fig 3.1 that the infinitesimal $i \epsilon$ is not needed to shift the poles, thus the Wick rotated propagator automatically incorporates the time ordered property.

Euclidean propagators first become especially useful when doing loop level calculations in the Feynman diagram perturbation scheme. Not only do they have the time-ordered property built in, but by going to Euclidean space the underlying symmetry group changes from $S O(3,1)$ to $S O(4)$. This facilitates many of the integrations encountered in the loop diagram expressions and can be made rigorous via the Osterwalder-Schrader conditions [10].

\subsubsection{Finite Temperature}

Euclidean propagators also become particularly useful for finite temperature quantum field theories. At a finite temperature $T=1 / \beta$ the expectation value of an operator $\langle\hat{A}\rangle$ becomes $\langle\hat{\rho} \hat{A}\rangle$, where the density operator at thermal equilibrium is

$$
\hat{\rho}=\frac{1}{Z_{\mathrm{G}}} \exp (-\beta(\hat{H}-\mu \hat{N})) .
$$


Here $Z_{\mathrm{G}}$ is the grand-canonical partition function,

$$
Z_{\mathrm{G}}=\operatorname{Tr}[\exp (-\beta(\hat{H}-\mu \hat{N}))]
$$

$\hat{H}$ is the Hamiltonian operator, c.f. eq. 3.3 and eq. 3.23), $\mu$ the chemical potential, and $\hat{N}$ the number operator, eq. 3.20 . Using this thermal average one can show that the Euclidean propagators must be periodic in imaginary time with period $\beta$, that is,

$$
D_{\mathrm{E}}\left(\tau, \mathbf{x} ; \tau^{\prime}, \mathbf{x}^{\prime}\right)=D_{\mathrm{E}}\left(\tau+\beta, \mathbf{x} ; \tau^{\prime}, \mathbf{x}^{\prime}\right)
$$

Therefore a finite temperature quantum field theory in flat space corresponds to a statistical quantum mechanical system with periodic boundary conditions, that is, on the circle cross Euclidean three space, $S \times \mathbb{R}^{3}$. This is made rigorous with the Kubo, Martin, Schwinger (KMS) condition [11, 12].

\subsubsection{Euclidean Path Integral and the Partition Func- tion}

The Wick rotated Euclidean path integral, ignoring the source term, is given by,

$$
Z=\int \mathrm{D}[\phi] \exp \left(-S_{\mathrm{E}}[\phi]\right)
$$

where, $S_{\mathrm{E}}[\phi]$, is the Wick rotated action, obtained from eq. 3.30 in the limit $t \rightarrow-i t$. This rotation picks up a factor of $-i$ from the change in determinant of the Minkowski metric. That is, $1=\sqrt{-g_{L}} \rightarrow \sqrt{-g_{E}}=-i$. This Euclidean path integral eq. (3.39) has the advantage of being mathematically well defined as a Wiener integral. The techniques presented in the previous section for the Lorentzian path integral are best understood in a formal sense.

One further consequence of the correspondence of finite temperature quantum field theory and statistical mechanics is that the path integral eq. 3.30 when Wick rotated and subject to periodic boundary conditions becomes the partition function of the statistical system, that is, ignoring the chemical 
potential $\mu$,

$$
Z_{\mathrm{G}}=\operatorname{Tr}[\exp (-\beta \hat{H})]=\int \mathrm{D}[\phi] \exp \left(-\int_{0}^{\beta} \mathrm{d} \tau \int \mathrm{d}^{3} x \mathscr{L}_{\mathrm{E}}\right)
$$

This is apparent since the Euclidean Lagrangian density,

$$
\begin{aligned}
\mathscr{L}_{E} \sqrt{g_{E}} & =-i \lim _{t \rightarrow-i t}\left[\mathscr{L} \sqrt{-g_{L}}\right] \\
& =\left(\frac{1}{2}\left[\left(\partial_{t} \phi\right)^{2}+(\nabla \phi)^{2}+m^{2} \phi^{2}\right]+V_{I}(\phi)\right) \sqrt{g_{E}}
\end{aligned}
$$

corresponds exactly to the interacting Hamiltonian. Thus, a system which is periodic in imaginary time $\tau$ with period $\beta$ corresponds to a thermal system with temperature $\beta^{-1}$. In the zero temperature limit, $\beta \rightarrow \infty$, the standard Wick rotated QFT can be recovered.

In the next chapter we look at the generalisation QFT to a generic curved spacetime. It will be seen that without a unique time direction and time-like Killing vector many of the methods presented here break down. 


\section{Chapter 4}

\section{Applications of Quantum Field Theory in Curved Spacetime}

In this chapter we generalise the concepts presented in Chapter 3 to a fixed curved spacetime background. In this process we build up to the derivation of Hawking radiation from black holes. We present two routes to this result; the first is the original derivation by Hawking [13] based on fields propagating near a collapsing body which forms a horizon, while the second employs a Wick rotation and the methods of Euclidean quantum field theory. Once more we consider a scalar field for simplicity. We refer the reader again to Birrell and Davies [7] for more details.

\subsection{Extension to Curved Space}

The Lagrangian of the free scalar field, $\phi$, eq. (3.1), is modified as the flat space metric, $\eta_{a b}$, becomes an arbitrary metric, $g_{a b}$, which is a solution to the Einstein field equations (2.37). Further the field is able to couple to the Ricci scalar of the spacetime, that is,

$$
\mathscr{L}=-\frac{1}{2}\left(\partial^{a} \phi \partial_{a} \phi+m^{2} \phi^{2}+\xi R\right)
$$


where, $\xi$, is a numerical coupling constant. Variation of the Lagrangian leads to the equations of motion

$$
-\nabla^{a} \nabla_{a} \phi+\left(m^{2}+\xi R\right) \phi=0 .
$$

Here $\nabla_{a}$ is the covariant derivative with Christoffel connection. Now the inner product eq. (3.7) can be generalised to,

$$
\left(\phi_{1}, \phi_{2}\right)=-i \int_{\Sigma}\left(\phi_{1}(x) \mathcal{L}_{n} \phi_{2}^{*}(x)-\mathcal{L}_{n}\left[\phi_{1}(x)\right] \phi_{2}^{*}(x)\right) \mathrm{d} \Sigma
$$

where $\mathcal{L}_{n}$ is the Lie derivative along $n^{a}$, a future directed time-like vector orthogonal to the space-like hypersurface $\Sigma$. It can be shown that this inner product is independent of the choice of hypersurface [14].

For eq. (4.2) it is still possible to find an orthonormal basis, $u_{i}, u_{i}^{*}$, such that,

$$
\left(u_{i}, u_{j}\right)=\delta_{i j}, \quad\left(u_{i}^{*}, u_{j}^{*}\right)=-\delta_{i j}, \quad\left(u_{i}, u_{j}^{*}\right)=0
$$

And using this the field maybe expanded,

$$
\phi(x)=\sum_{i}\left[a_{i} u_{i}(x)+a_{i}^{\dagger} u_{i}^{*}(x)\right] .
$$

One then can quantise the field by again promoting to operators in a Hilbert space $a_{i}^{(\dagger)} \rightarrow \hat{a}_{i}^{(\dagger)}$ and introducing the commutation relations c.f. eq. 3.12,

$$
\left[\hat{a}_{i}, \hat{a}_{j}\right]=0, \quad\left[\hat{a}_{i}^{\dagger}, \hat{a}_{j}^{\dagger}\right]=0, \quad\left[\hat{a}_{i}, \hat{a}_{j}^{\dagger}\right]=\delta_{i j}
$$

This process however is not unique and can have physical implications as we shall see. Propagators for eq. (4.2) as Green functions can be obtained via the relation,

$$
\left[-\nabla^{a} \nabla_{a} \phi+\left(m^{2}+\xi R\right)\right] D\left(x, x^{\prime}\right)=\sqrt{-g} \delta^{4}\left(x-x^{\prime}\right)
$$

However, in order to specify the time ordered nature of the causal Feynman propagator one needs to impose boundary conditions. These are not so simple in the curved space setting as choosing a contour of integration. We shall return to this when we consider the generalisation of Wick rotation in Chapter 6. 


\subsubsection{Quantum Vacuum States and Particles}

Now the expansion of $\phi(x)$ in terms of $u_{i}(x)$ defines a quantum vacuum state for the operators $\hat{a}_{i}^{(\dagger)}$ by,

$$
\hat{a}_{i}|0\rangle=0, \quad \text { for all } i \text {. }
$$

However, a generic metric $g_{a b}$ does not have the same symmetries as the flat Minkowski space. In particular there is not necessarily a time-like Killing vector, $\partial_{t}$, which was used in Chapter 3 to define positive eigen-frequency modes, $u_{\omega}$, and a quantum vacuum state, $|0\rangle$, which was invariant under Lorentz transformations.

This means that, in the curved space setting, a second observer can define a different mode expansion based on new orthonormal modes $\bar{u}_{i}, \bar{u}_{i}^{*}$,

$$
\hat{\phi}(x)=\sum_{i}\left[\hat{b}_{i} \bar{u}_{i}(x)+\hat{b}_{i}^{\dagger} \bar{u}_{i}^{*}(x)\right]
$$

which defines a new vacuum state $|\overline{0}\rangle$,

$$
\hat{b}_{i}|\overline{0}\rangle=0, \quad \text { for all } i \text {. }
$$

Since each mode expansion forms a basis they can be written as a linear combination of each other,

$$
\begin{aligned}
& \bar{u}_{i}=\sum_{j}\left[\alpha_{i j} u_{j}+\beta_{i j} u_{j}^{*}\right], \\
& u_{i}=\sum_{j}\left[\alpha_{i j}^{*} \bar{u}_{j}-\beta_{i j} \bar{u}_{j}^{*}\right],
\end{aligned}
$$

where, $\alpha_{i j}=\left(\bar{u}_{i}, u_{j}\right)$, and, $\beta_{i j}=-\left(\bar{u}_{i}, u_{j}^{*}\right)$, are known as Bogoliubov coefficients. These Bogoliubov coefficients satisfy the following properties,

$$
\begin{aligned}
\sum_{k}\left[\alpha_{i k} \alpha_{j k}^{*}-\beta_{i k} \beta_{j k}^{*}\right] & =\delta_{i j} \\
\sum_{k}\left[\alpha_{i k} \beta_{j k}-\beta_{i k} \alpha_{j k}\right] & =0
\end{aligned}
$$

Equating the field expansions eq. 4.5 and eq. 4.9) and using the orthonor- 
mality of the modes, the operators can be related to each other via,

$$
\begin{aligned}
& \hat{a}_{i}=\sum_{j}\left[\alpha_{i j} \hat{b}_{j}+\beta_{i j}^{*} \hat{b}_{j}^{\dagger}\right], \\
& \hat{b}_{i}=\sum_{j}\left[\alpha_{i j}^{*} \hat{a}_{j}-\beta_{i j}^{*} \hat{a}_{j}^{\dagger}\right] .
\end{aligned}
$$

Thus it is clear that number of particles the second observer measures in the original vacuum $|0\rangle$ is non-zero. That is,

$$
\langle 0|\hat{\bar{N}}| 0\rangle=\left\langle 0\left|\hat{b}_{i}^{\dagger} \hat{b}_{i}\right| 0\right\rangle=\sum_{j}\left|\beta_{i j}\right|^{2} .
$$

If, however, there is a time-like Killing vector, $\partial_{t}$, used to define both positive frequency modes, $u_{\omega}$, and, $\bar{u}_{\omega}$, then $\beta_{\omega \omega^{\prime}}=0$ and they share a common vacuum. We shall see the consequences of eq. (4.17) in the next two sections.

\subsection{The Unruh Effect}

Strictly speaking the Unruh effect [15, 16, 17] occurs in Minkowski spacetime. However, it serves as a useful example of the influence of non-inertial observers on the detection of particles and as a way to understand the Hawking radiation process.

Consider an accelerating observer with a constant acceleration, $a$, in flat Minkowski spacetime. We shall call them a Rindler observer who uses proper time along their world line as a time coordinate. We will compute the number of particles the Rindler observer measures. To simplify the calculation we shall work in $(1+1)$ spacetime.

An inertial observer in Minkowski space has metric c.f. eq (2.4),

$$
\mathrm{d} s^{2}=-\mathrm{d} t^{2}+\mathrm{d} x^{2}=-\mathrm{d} \bar{u} \mathrm{~d} \bar{v}
$$

where, $\bar{u}=t-x$, and $\bar{v}=t+x$ are ingoing and outgoing null coordinates. The Rindler observer has metric,

$$
\mathrm{d} s^{2}=-e^{a(v-u)} \mathrm{d} u \mathrm{~d} v=e^{2 a X}\left(-\mathrm{d} T^{2}+\mathrm{d} X^{2}\right)
$$


where $2 X=u-v$ and $2 T=u+v$. The two observers' coordinates are related by the transformation,

$$
v=\frac{1}{a} \ln \bar{v}, \quad u=\frac{1}{a} \ln (-\bar{u})
$$

It can be seen that the Rindler coordinates only represent a wedge of Minkowski spacetime, since the accelerating observer never exceeds the speed of light. That is, the observer is bounded by the $x= \pm t$ surfaces which in analogy with black holes we denote as the horizons $\mathcal{H}^{-}, \mathcal{H}^{+}$. On these the time-like Killing vector $\partial_{T}$ becomes null.

Consider now a free massless scalar field. In the global inertial coordinates, one can define the basis of positive frequency ingoing and outgoing modes, $f_{\omega}, j_{\omega}$ respectively by,

$$
f_{\omega}=\frac{1}{\sqrt{2 \pi \omega}} e^{-i \omega \bar{v}}, \quad j_{\omega}=\frac{1}{\sqrt{2 \pi \omega}} e^{-i \omega \bar{u}} .
$$

The scalar field is then given by,

$$
\hat{\phi}=\int \mathrm{d} \omega\left(\hat{a}_{\omega} f_{\omega}+\hat{a}_{\omega}^{\dagger} f_{\omega}^{*}+\hat{d}_{\omega} j_{\omega}+\hat{d}_{\omega}^{\dagger} j_{\omega}^{*}\right)
$$

where the mode operators $\hat{a}, \hat{d}$ annihilate the global inertial vacuum as in Chapter 3 ,

$$
\hat{a} \mid 0, \text { in }\rangle=0=\hat{d} \mid 0, \text { in }\rangle, \quad \text { for all } \omega>0 .
$$

We now assume the field is in this vacuum state.

Due to the conformal invariance of the $(1+1) \mathrm{D}$ wave equation for a massless scalar field, the Rindler observer also has a basis of plane wave modes in their coordinates,

$$
q_{\omega}=\frac{1}{\sqrt{2 \pi \omega}} e^{-i \omega v}, \quad p_{\omega}=\frac{1}{\sqrt{2 \pi \omega}} e^{-i \omega u} .
$$

Thus on the Rindler wedge the field can also be expanded as,

$$
\hat{\phi}=\int \mathrm{d} \omega\left(\hat{b}_{\omega} p_{\omega}+\hat{b}_{\omega}^{\dagger} p_{\omega}^{*}+\hat{c}_{\omega} q_{\omega}+\hat{c}_{\omega}^{\dagger} q_{\omega}^{*}\right)
$$

where the operator, $\hat{b}_{\omega}^{\dagger}\left(\hat{c}_{\omega}^{\dagger}\right)$, creates ingoing (outgoing) Rindler particles. The calculation begins by assuming we have an outward propagating Rindler 
wave packet on future null infinity, $\mathscr{I}^{+}$, i.e. $\phi \rightarrow p_{\omega}$. We want to find the form of the field in the far past, which consists of $\mathcal{H}^{-}$and $\mathscr{I}^{-}$. However as spacetime is flat there is no scattering of the scalar field and thus only travels from $\mathscr{I}^{+}$to $\mathcal{H}^{-}$. Thus we need to find the Bogoliubov coefficients on $\mathcal{H}^{-}$,

$$
\alpha_{\omega \omega^{\prime}}=\left(p_{\omega}, j_{\omega^{\prime}}\right)_{\mathcal{H}^{-}}, \quad \beta_{\omega \omega^{\prime}}=-\left(p_{\omega}, j_{\omega^{\prime}}^{*}\right)_{\mathcal{H}^{-}}=-i \alpha_{\omega-\omega^{\prime}}
$$

Now we have,

$$
\begin{aligned}
\alpha_{\omega \omega^{\prime}} & =-i \int_{-\infty}^{0} \mathrm{~d} \bar{u}\left(p_{\omega} \partial_{\bar{u}} j_{\omega^{\prime}}^{*}-\partial_{\bar{u}} p_{\omega} j_{\omega}^{*}\right) \\
& =\frac{1}{2 \pi \sqrt{\omega \omega^{\prime}}} \int_{-\infty}^{0} \mathrm{~d} \bar{u}\left(\omega^{\prime}-\frac{\omega}{a \bar{u}}\right) e^{i \omega^{\prime} \bar{u}} e^{i \frac{\omega}{a} \ln (-\bar{u})} \\
& =\frac{i}{\pi \sqrt{\omega \omega^{\prime}}}(i \omega)^{-i \frac{\omega}{a}} \Gamma\left(1+i \frac{\omega}{a}\right)
\end{aligned}
$$

In the last line we have used the Gamma function, $\Gamma(s)=\int_{0}^{\infty} e^{-z} z^{s-1}$, in a distributional/generalised function sense, and the property $\Gamma(s+1)=s \Gamma(s)$. Using eq. (4.26), the $\beta_{\omega \omega^{\prime}}$ coefficients can be obtained from eq. (4.27), by $\omega^{\prime} \rightarrow-\omega^{\prime}$. Due to the prefactor $(i \omega)^{-i \frac{\omega}{a}}$ there is a logarithmic singularity at $\omega^{\prime}=0$. So one needs to analytically continue this expression anticlockwise around this singularity [13. This yields the relation,

$$
\left|\alpha_{\omega \omega^{\prime}}\right|=\exp \left(\frac{\pi \omega}{a}\right)\left|\beta_{\omega \omega^{\prime}}\right| .
$$

Eq. 4.28 can be used to find the number of particles the Rindler observer detects. Using the properties in eq. 4.13, we have,

$$
\int \mathrm{d} \omega^{\prime}\left(\left|\alpha_{\omega \omega^{\prime}}\right|^{2}-\left|\beta_{\omega \omega^{\prime}}\right|^{2}\right)=\delta(0)
$$

Therefore combining eq. 4.28 and eq. (4.29),

$$
\left\langle 0\left|\hat{\bar{N}}_{\omega}\right| 0\right\rangle=\int \mathrm{d} \omega^{\prime}\left|\beta_{\omega \omega^{\prime}}\right|^{2}=\left[\exp \left(\frac{2 \pi \omega}{a}\right)-1\right]^{-1} \delta(0) .
$$

The $\delta(0)$ term is the consequence of considering plane waves which extend 
over infinite volume. Hence the number of particles per unit volume is,

$$
\hat{n}_{\omega}=\left[\exp \left(\frac{2 \pi \omega}{a}\right)-1\right]^{-1}
$$

which represents a thermal spectrum with temperature,

$$
T=\frac{a}{2 \pi}
$$

In the case of the Rindler observer it can be shown that the thermal spectrum they measure uses the energy of the accelerating detector to create particles in the vacuum. This is a toy model which requires a constant acceleration over an infinite time. Naturally, the response to a realistic acceleration would be much messier in the details.

\subsection{Hawking Radiation}

We now turn to the previously anticipated Hawking effect. The details of the calculation will turn out to be very similar to the Unruh effect of the previous section.

Early work by Bardeen, Cater, and Hawking in the 1970s suggested that black holes obey a set of rules heuristically very similar to the laws of thermodynamics [18]. With surface gravity, $\kappa$, playing the role of temperature and surface area of the horizon, $A$, playing the role of entropy. Beckenstein had also earlier proposed black holes have entropy proportional to their surface area [19]. This turned out to be more than just a correspondence when in 1974 Hawking showed that quantum field theoretical effects in curved spacetime indeed lead to a black holes having a temperature proportional to their surface gravity, $T_{\mathrm{H}}=\kappa / 2 \pi[13,20]$.

\subsubsection{Original Derivation}

We present here the original derivation based on a collapsing spherically symmetric body which forms a black hole. We refer the reader to Hawking [13] and Birrell and Davies [7] for more details. This process is shown by a Penrose-Carter diagram in Fig. 4.1. 


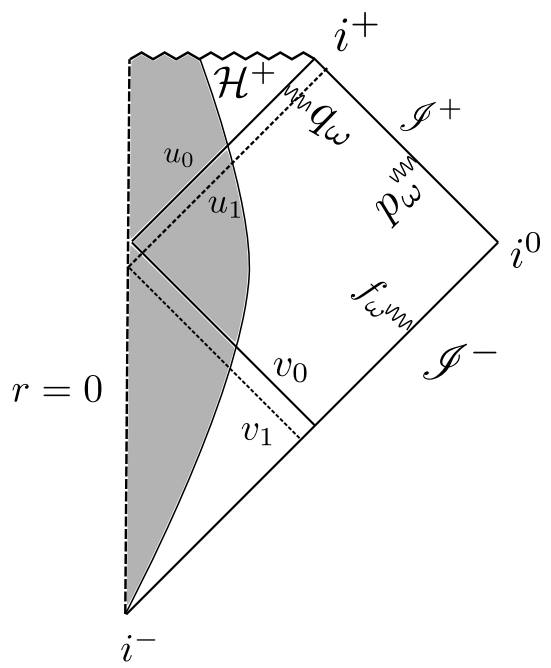

Figure 4.1: The conformal Penrose-Carter diagram for a collapsing spherically symmetric body. The massive body is shown in shaded grey. Once its radius drops below $r=2 M$ a horizon, $\mathcal{H}^{+}$, forms.

In the quasi-static situation after the collapse the metric is given by the Schwarzschild solution eq. 2.40). It is useful to consider the Kruskal-Szekeres form of the metric eq. 2.43,

$$
d s^{2}=-\frac{2 M}{r} e^{-r / 2 M} \mathrm{~d} U \mathrm{~d} V+r^{2}\left(\mathrm{~d} \theta^{2}+\sin ^{2} \theta \mathrm{d} \varphi^{2}\right) .
$$

For a spherically symmetric wave equation we can always decompose the solution into angular momentum modes $\phi_{m, \ell, \omega}$, which is what we consider henceforth. Here $m$ labels the azimuthal angular momentum number and $\ell$ the principle angular momentum number. We assume further a minimally coupled field, that is $\xi=0$ in eq. 4.1.

In the distance null past, that is, on $\mathscr{I}^{-}$, the spacetime is approximately flat Minkowski space as the strong gravitational effects are near the spherical body. This means modes can be expanded in positive frequency solutions, $f_{\omega}$, to the flat space wave equation with respect to Minkowski time,

$$
f_{\omega} \rightarrow e^{-i \omega v}, \quad \text { on } \mathscr{I}^{-}
$$

where $v$ is the ingoing null coordinate for flat space. 
Further we can assume a vacuum state $\mid 0$, in $\rangle$, defined by,

$$
\left.\hat{a}_{\omega} \mid 0, \text { in }\right\rangle=0, \quad \text { for all } \omega>0
$$

As before the field can be expanded in terms of the creation (annihilation) operators, $\hat{a}_{\omega}^{(\dagger)}$,

$$
\phi=\int \mathrm{d} \omega\left(\hat{a}_{\omega} f_{\omega}+\hat{a}_{\omega}^{\dagger} f_{\omega}^{*}\right)
$$

At late times modes must defined on both $\mathscr{I}^{+}$and the horizon $\mathcal{H}^{+}$, call these $p_{\omega}$ and $q_{\omega}$ respectively. We require the out-states to be solutions to the wave equation with the asymptotic form such that,

$$
p_{\omega} \rightarrow e^{-i \omega u}, \quad \text { on } \mathscr{I}^{+} \text {. }
$$

We note, the choice of modes $q_{\omega}$ on the horizon $\mathcal{H}^{+}$does not affect the result of the calculation [13]. Thus the field can also be expanded,

$$
\phi=\int \mathrm{d} \omega\left(\hat{b}_{\omega} p_{\omega}+\hat{b}_{\omega}^{\dagger} p_{\omega}^{*}+\hat{c}_{\omega} q_{\omega}+\hat{c}_{\omega}^{\dagger} q_{\omega}^{*}\right)
$$

Considering a mode of frequency $\omega$ propagating from $\mathscr{I}^{-}$towards the horizon. The wave essentially scatters in two parts. A portion $1-T_{m, l}(\omega)$ scatters off the gravitational field and propagates out to $\mathscr{I}^{+}$. The other fraction $T_{m, l}(\omega)$ travels parallel to the horizon $\mathcal{H}^{-}$and is absorbed. This fraction leads to particle production and so we separate the modes in to these two parts; $f_{\omega}=f_{\omega}^{(1)}+f_{\omega}^{(2)}$ and likewise for $p_{\omega}, q_{\omega}$. The Bogoliubov coefficients can be expressed with this decomposition as,

$$
\begin{aligned}
\alpha_{\omega \omega^{\prime}} & =\alpha_{\omega \omega^{\prime}}^{(1)} \delta_{\omega \omega^{\prime}}+\alpha_{\omega \omega^{\prime}}^{(2)}, \quad \beta_{\omega \omega^{\prime}}=\beta_{\omega \omega^{\prime}}^{(2)}, \\
T_{m, \ell}(\omega) & =\int \mathrm{d} \omega^{\prime}\left(\left|\alpha_{\omega \omega^{\prime}}^{(2)}\right|^{2}-\left|\beta_{\omega \omega^{\prime}}^{(2)}\right|^{2}\right) .
\end{aligned}
$$

To obtain finite expressions for $T_{m, \ell}(\omega)$ in the above equation one can consider wave packets peaked about the frequency, $\omega$, which are square integrable; this does not change the key points of the calculation.

To evaluate the Bogoliubov coefficients, consider the boundary conditions such that the field $\phi \rightarrow p_{\omega}$ as before. We then need to find expressions for this field on $\mathscr{I}^{-}$and decompose it into positive and negative frequency 
components. Now trace the mode backwards in time along a path, $\gamma$, from $\mathscr{I}^{+}$along the null-geodesic $u=u_{1}$ which passes near to the horizon $\mathcal{H}^{+}$which lies on $u=u_{0}$. It then passes out through the collapsing mass to $\mathscr{I}^{-}$along the geodesic $v_{1}$; these are shown in Fig. 4.1. Inward travelling modes with $v>v_{0}$ fall into the horizon which forms. The ray $\gamma$ is connected to $u_{0}$ and $v_{0}$ be a small geodesic deviation vector $\epsilon n^{a}$. By considering the null geodesics on the horizon and the geodesic equation in local inertial coordinates near the horizon, one can show that $\epsilon \propto \lambda$, where $\lambda$ is an affine parameter of the null generators of the horizon. Using eq. 2.13 and eq. 2.44), for the Schwarzschild metric, the affine parameter is given by,

$$
\lambda \propto e^{-\kappa u}=U
$$

where $\kappa=1 / 4 M$ is the surface gravity of the horizon. Near $\mathscr{I}^{-}$in the distant past, since spacetime is nearly flat $v_{1}$ is connected to $v_{0}$ by,

$$
v_{0}-v=\epsilon=C e^{-\kappa u}
$$

Thus using the boundary condition $\phi \sim^{-i \omega u}$ on $\mathscr{I}^{+}$on $\mathscr{I}^{-}$we have for $v_{1}<v_{0}$,

$$
\phi \sim e^{i \frac{\omega}{\kappa} \ln \left(\frac{v_{0}-v_{1}}{C}\right)} .
$$

Those modes with $v_{1}>v_{0}$ are zero on $\mathscr{I}^{-}$as they can not come from behind the horizon. Eq. (4.41) is comparable to the case of the Rindler observer in the previous section and thus leads the same relation for the Bogoliubov coefficients,

$$
\left|\alpha_{\omega \omega^{\prime}}\right|=e^{\frac{\pi \omega}{\kappa}}\left|\beta_{\omega \omega^{\prime}}\right|
$$

with $a \rightarrow \kappa$. Thus the number of particles detected is a thermal spectrum with Hawking temperature,

$$
T_{H}=\frac{\kappa}{2 \pi},
$$

similar to the previous situation but modified by the fraction of particles which scatter off the gravitational field. This fraction is called the greybody factor, $T_{\ell, m}(\omega)$. So the number of particles measured in a given mode is,

$$
\hat{n}_{\omega}=T_{\ell, m}(\omega)\left[\exp \left(\frac{2 \pi \omega}{\kappa}\right)-1\right]^{-1},
$$


This can be extended to a generic black hole and massless particle with spin, $s$, where the Hawking temperature is given by the surface gravity on the horizon of the generic black hole [13]. The study of greybody factors for a Schwarschild black hole forms the basis of the next chapter.

\subsubsection{Hawking Radiation via Wick Rotation}

There is another way to arrive at the thermal spectrum result of the last section via a Wick rotation [21]. The Schwarzschild geometry is very special in that it has a time-like Killing vector, $\partial_{t}$ and no rotation, i.e. is static. This allows one to analytically continue the time coordinate $t \rightarrow \tau=-i t$ and obtain a real valued Euclidean signature metric,

$$
\mathrm{d} s^{2}=\left(1-\frac{2 M}{r}\right) \mathrm{d} \tau^{2}+\left(1-\frac{2 M}{r}\right)^{-1} \mathrm{~d} r^{2}+r^{2}\left(\mathrm{~d} \theta^{2}+\sin ^{2} \theta \mathrm{d} \varphi^{2}\right) .
$$

This is valid outside the horizon $r=2 M$ where $t$ is a time-like coordinate. Near $r=2 M$ the pre-factor can be approximated,

$$
1-\frac{2 M}{r} \simeq 2 \kappa(r-2 M)+O\left([r-2 M]^{2}\right)
$$

where $\kappa=1 / 4 M$ is again the surface gravity. Now by the coordinate transformation $\kappa^{2} R^{2}=2 \kappa(r-2 M)$, we have $\mathrm{d} r \simeq \kappa R \mathrm{~d} R$. So defining the solid angle $\mathrm{d} \Omega^{2}=\mathrm{d} \theta^{2}+\sin ^{2} \theta \mathrm{d} \varphi^{2}$, the Euclidean metric takes the form,

$$
\mathrm{d} s^{2} \simeq R^{2} \kappa^{2} \mathrm{~d} \tau^{2}+\mathrm{d} R^{2}+(2 M)^{2} \mathrm{~d} \Omega^{2}
$$

We can, for simplicity, drop the angular part and consider only the 2D line element,

$$
\mathrm{d} s^{2} \simeq R^{2} \kappa^{2} \mathrm{~d} \tau^{2}+\mathrm{d} R^{2}
$$

These two terms represent the line element of a cone with $\tau$ the angular coordinate. If we require the analytically continued metric to be non singular at the horizon, $R=0$, then $\tau$ must be periodic with $\beta=2 \pi / \kappa$ to avoid a conical singularity [21]. Thus using the Euclidean methods in the previous section the periodic metric tensor field implies a thermal system with Hawking temperature $T_{\mathrm{H}}=\beta^{-1}=\kappa / 2 \pi$ for a Schwarzschild black hole. 
This is a very quick and simple way to obtain the Hawking temperature and is highly suggests that Euclidean techniques could have applications in a quantum theory of gravity. However the Wick rotation used requires the metric to be static and it is not clear if it can be generalised to arbitrary geometries or if indeed it makes any sense to do so. The process of generalised Wick rotation will be the focus of Chapter 6 .

We now continue with a study of the greybody factors that arise in the Hawking emission process. 


\section{Chapter 5}

\section{Greybody Factors}

The greybody factors which arise in the Hawking radiation process and influence the emission of particles from black holes are intimately related to the scattering of particles by the gravitational potential of the black hole.

In the first section of this chapter we present some of the important physical quantities from the emission process in terms of the greybody factors. In the second section we show that the problem of the greybody factors can be reduced to finding the transmission probabilities for a one dimensional (1D) Schrödinger type equation with Regge-Wheeler potential. We present the conventional methods of analysis via asymptotically plane wave solutions with some analytic results for low frequencies. The third and fourth sections develop a new method for their calculation based on a transfer matrix approach and path ordered exponentials and present the results of the numerical implementation of this method. Finally the last section outlines some of the physical implications of the greybody factors and the possible extensions to this work.

\subsection{Emission Rates from Schwarzschild Black Holes}

For simplicity we consider massless fields of spin, $s \in 0,1,2$, (i.e. scalars, photons, and gravitons), in the Schwarzschild black hole spacetime with metric 
as in eq. 2.40

$$
\mathrm{d} s^{2}=-\left(1-\frac{2 M}{r}\right) \mathrm{d} t^{2}+\left(1-\frac{2 M}{r}\right)^{-1} \mathrm{~d} r^{2}+r^{2}\left(\mathrm{~d} \theta^{2}+\sin ^{2} \theta \mathrm{d} \varphi^{2}\right)
$$

We now derive some of the important physical quantities from the Hawking process that require explicit knowledge of the greybody factors. The emission rate, $d^{2} N_{s}(\omega) / d t d \omega$, represents the total probability for an emission per unit time, per unit frequency of a particle of spin $s$, and frequency, $\omega$. It is given by the sum over the greybody factor (transmission probability), $T_{\ell, s}(\omega)$ (see eq. (4.44)), for each principal and azimuthal angular momentum mode, (which in the case of spherical symmetry are equi-probable), multiplied by the probability for a particle to be in a given mode $P_{\ell, s}(\omega)$. See references [13, 20, 22, 23. That is,

$$
\frac{d^{2} N_{s}(\omega)}{d t d \omega}=\sum_{\ell=s}^{\infty}(2 \ell+1) T_{\ell, s}(\omega) P_{\ell, s}(\omega)
$$

For integer spin particles the probability for a the particle to be in a particular mode is given by the Bose-Einstein distribution,

$$
P_{\ell, s}(\omega)=\frac{g}{2 \pi} \frac{1}{\exp \left(\omega / T_{H}\right)-1},
$$

and $g$ is the number of polarizations for a given spin $s$. Recall from eq. 4.43, $T_{H}=\kappa / 2 \pi$ is the Hawking temperature which for a Schwarzschild black hole is $1 / 8 \pi M$. Eq. (5.1) represents the rate of the emission of particles. Each particle carries a quantum of energy, $\omega$, and so the energy emission is given by

$$
\frac{d^{2} E_{s}(\omega)}{d t d \omega}=\omega \frac{d^{2} N_{s}(\omega)}{d t d \omega}
$$

Another physically important quantity is the cross-section, $\sigma(\omega)$, which represents an effective area that embodies the likelihood of a particle to be scattered, i.e. deflected, by the black hole. This is intimately related to the probability of transmission through the potential barrier [23],

$$
\sigma_{s}(\omega)=\pi \omega^{-2} \sum_{\ell=s}^{\infty}(2 \ell+1) T_{\ell, s}(\omega)
$$


In the high frequency limit, $M \omega \gg 1$, this approaches the classical geometric optics cross-section $\sigma_{\infty}=27 \pi M^{2}$ [4, 23]. This can be used to define the dimensionless measure, $S(x)$, of the cross-section,

$$
S(x)=\frac{\sigma(x)}{\sigma_{\infty}}=\frac{1}{27 x^{2}} \sum_{\ell=s}^{\infty}(2 \ell+1) T_{\ell, s}(x),
$$

where $x=M \omega$. Eq. (5.5) can then be used to rewrite eq. (5.1) and eq. 5.3) in dimensionless form,

$$
M \frac{d^{2} E_{s}(x)}{d t d \omega}=x \frac{d^{2} N_{s}(x)}{d t d \omega}=\frac{g}{2 \pi} \frac{27 x^{3} S(x)}{\exp (8 \pi x)-1} .
$$

With these physical quantities the radiation process can be well characterised. In the next section we present the development of the Regge-Wheeler potential which governs the scattering process.

\subsection{Regge-Wheeler Equation}

In the early 1970s Price [24, 25] showed that scalar, photon and graviton fields are governed by a complete set of dynamical gauge-invariant functions $\Phi^{(s)}$ which satisfy the wave equation in curved space. These can be shown to reduce to a 1D Schrödinger type equation with the Regge-Wheeler potential. The greybody factors are then related to the probability of transmission found by analysing these wave equations. We present in detail the derivation for a scalar field and show the results for the photon and graviton, see the references [26, 27, 28, 29, 30] for more details on these.

\subsubsection{Scalar}

As in the previous chapter we consider a minimally coupled massless scalar field $\Phi^{(0)}\left(x^{a}\right)$ in the Schwarzschild spacetime which satisfies,

$$
\nabla_{a} \nabla^{a} \Phi^{(0)}=0
$$

where $\nabla_{a}$ is the covariant derivative with the Christoffel connection associated with the Schwarzschild metric $g_{a b}$ given by eq. 2.40). Eq. (5.7) can be 
written purely in terms of partial derivatives as,

$$
\frac{1}{\sqrt{-g}} \partial_{a}\left[\sqrt{-g} g^{a b} \partial_{b} \Phi^{(0)}\left(x^{a}\right)\right]=0
$$

Here, $g=-r^{4} \sin ^{2} \theta$, is the determinant of the metric $g_{a b}$. Defining, $f(r) \equiv$ $1-2 M / r$, this becomes,

$-f^{-1} \partial_{t}^{2} \Phi^{(0)}+\frac{1}{r^{2}} \partial_{r}\left[r^{2} f \partial_{r} \Phi^{(0)}\right]+\frac{1}{r^{2} \sin \theta} \partial_{\theta}\left[\sin \theta \partial \Phi^{(0)}\right]+\frac{1}{r^{2} \sin ^{2} \theta} \partial_{\varphi}^{2} \Phi^{(0)}=0$.

As a result of the spherical symmetry of the Schwarzschild solution the last two terms in eq. (5.9) can be recognised as part of the 3D flat-space Laplacian in spherical coordinates. Thus expanding in terms of spherical harmonics $Y_{\ell, m}(\theta, \varphi)$ we take as an ansatz,

$$
\Phi^{(0)}(t, r, \theta, \phi)=\sum_{\ell=0}^{\infty} \sum_{m=-\ell}^{\ell} \frac{\Psi_{\ell}(t, r)}{r} Y_{\ell, m}(\theta, \varphi),
$$

where, $\ell$ is the principal angular momentum number. There are $2 \ell+1$ azimuthal modes for each principle angular momentum mode. The remaining function $\Psi_{\ell}(r, t)$ satisfies the equation,

$$
-\partial_{t}^{2} \Psi_{\ell}(t, r)+f \partial_{r}\left[f \partial_{r} \Psi_{\ell}\right]=\frac{f}{r}\left(\frac{\ell(\ell+1)}{r}+\partial_{r} f\right) \Psi_{\ell}
$$

Changing variables to the tortoise coordinate, $r_{*}$, which is related to the radial coordinate $r$ implicitly by,

$$
\frac{d r_{*}}{d r}=f^{-1}=\frac{1}{1-\frac{2 M}{r}}
$$

and assuming harmonic time dependence,

$$
\Psi_{\ell}(r, t)=\left(A(\omega) e^{+i \omega t}+B(\omega) e^{-i \omega t}\right) \psi_{\ell}(r)
$$

eq. 5.11 becomes,

$$
-\frac{d^{2} \psi_{\ell}}{d r_{*}^{2}}+V\left(r_{*}\right) \psi\left(r_{*}\right)=\omega^{2} \psi_{\ell}\left(r_{*}\right)
$$


with potential,

$$
V\left(r_{*}\right)=\left[1-\frac{2 M}{r\left(r_{*}\right)}\right]\left[\frac{\ell(\ell+1)}{r\left(r_{*}\right)^{2}}+\frac{2 M}{r\left(r_{*}\right)^{3}}\right] .
$$

$r$ and $r_{*}$ are related by $r_{*}=r+2 M \ln (r / 2 M-1)$ as in chapter 2, This can be inverted explicitly

$$
r\left(r_{*}\right)=2 M\left[1+\mathrm{W}\left(\exp \left(\frac{r_{*}}{2 M}-1\right)\right)\right],
$$

where $\mathrm{W}(x)$ is the Lambert $\mathrm{W}$ function defined by [31]

$$
\mathrm{W} e^{\mathrm{W}}=x
$$

This potential is shown below in Fig. (5.1). Note the increasing potential height with increasing angular momentum number.

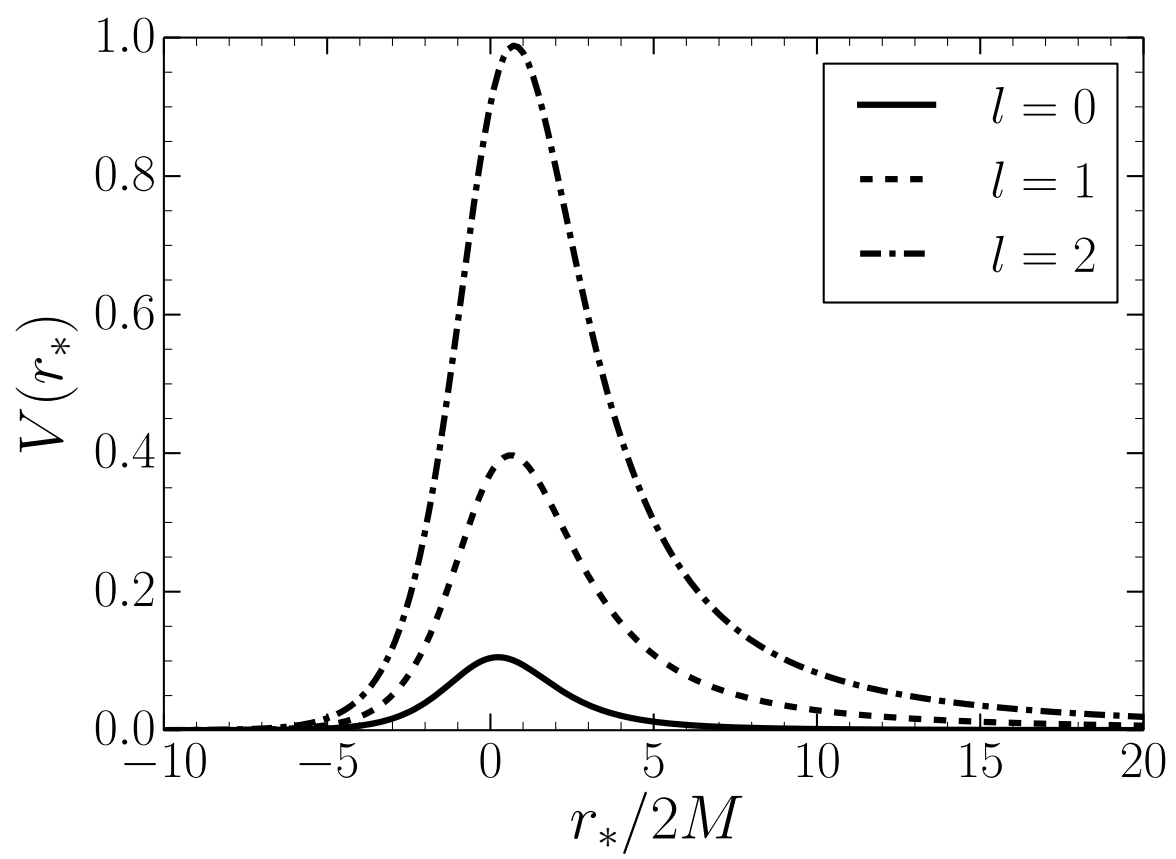

Figure 5.1: The potential barrier (in units of $(2 M)^{2}$ ) for a scalar scattered by a Schwarzschild black hole, for increasing angular momentum $\ell$.

Since the potential vanishes in the limit $r_{*} \rightarrow \pm \infty$ the solutions to eq. (5.14) must asymptote to freely propagating plane waves in these regions. Therefore, in a similar method to the derivation of Hawking radiation, we can 
choose to analyse incoming modes from the distant past, $\mathscr{I}^{-}$, which are normalised to unity, which are then scattered by black hole and make it out to the distant future $\mathscr{I}^{+}$. Due to scattering by the gravitational potential a fraction of these become outgoing $A_{\text {out }}^{(\ell, 0)}$ and a fraction remain ingoing $A_{\text {in }}^{(\ell, 0)}$. That is we consider solutions obeying the asymptotic behaviour

$$
\psi_{\ell}^{(0)}\left(r_{*}\right) \sim\left\{\begin{array}{ll}
e^{-i \omega r_{*}} & r_{*} \rightarrow-\infty \\
A_{\mathrm{out}}^{(\ell, 0)} e^{i \omega r_{*}}+A_{\mathrm{in}}^{(\ell, 0)} e^{-i \omega r_{*}} & r_{*} \rightarrow+\infty
\end{array} .\right.
$$

From this the transmission probabilities can be obtained

$$
T=1-\left|\frac{A_{\mathrm{out}}^{(\ell, 0)}}{A_{\mathrm{in}}^{(\ell, 0)}}\right|^{2}
$$

Next we present results for the photon and graviton cases and highlight the similarities.

\subsubsection{Photon}

The scattering of the electromagnetic field, i.e. photons, can be analysed by solving the source free Maxwell equations in the Schwarzschild spacetime,

$$
\begin{gathered}
\nabla_{a} F_{b c}=0, \\
\epsilon^{a b c d} \nabla_{b} F_{c d}=0,
\end{gathered}
$$

where $F_{a b}=\nabla_{a} A_{b}-\nabla_{b} A_{a}$ is the electromagnetic field tensor and $\epsilon^{a b c d}$ is the totally antisymmetric Levi-Civita tensor. Using the conformal invariance of eq. 5.20a and eq. 5.20b), and following reference [28, 29], the electromagnetic four-potential can be constructed as

$$
A_{a}=\left(u^{b} v_{a}-v^{b} u_{a}\right) \partial_{b} \Phi_{\mathrm{I}}^{(1)}+\epsilon_{a}^{b c d} u_{c} v_{d} \partial_{b} \Phi_{\mathrm{II}}^{(1)}
$$

\footnotetext{
${ }^{1}$ An alternative derivation may be found in 30 .
} 
where $v^{a}=(0,1,0,0), u^{a}=(1,0,0,0)$. The scalar fields $\Phi_{\mathrm{I}}^{(1)}, \Phi_{\mathrm{II}}^{(1)}$ are two independent solutions which are expanded in terms of spherical harmonics

$$
\Phi_{\mathrm{I}(\mathrm{II})}^{(1)}=\sum_{\ell=1}^{\infty} \sum_{m=-\ell}^{\ell} \Psi_{\ell}^{I(I I)}(t, r) Y_{\ell, m}(\theta, \varphi)
$$

where $\Psi_{\ell}^{I}(r, t), \Psi_{\ell}^{I I}(r, t)$, are independent solutions to a 1D type Schrödinger equation (i.e. eq. (5.14) ) now with the potential

$$
V\left(r_{*}\right)=\left[1-\frac{2 M}{r\left(r_{*}\right)}\right]\left[\frac{\ell(\ell+1)}{r\left(r_{*}\right)^{2}}\right] .
$$

As before, $\ell$ is the angular momentum number, but now only $\ell \geq 1$ modes are radiative. The transmission probabilities can found as before by considering solutions with the asymptotic behaviour given by eq. (5.18).

Fig. (5.2) below is a plot of this potential. Note the increase in barrier height as compared to the scalar but the overall similarity in shape. Once again the barrier increases with angular momentum $\ell$.

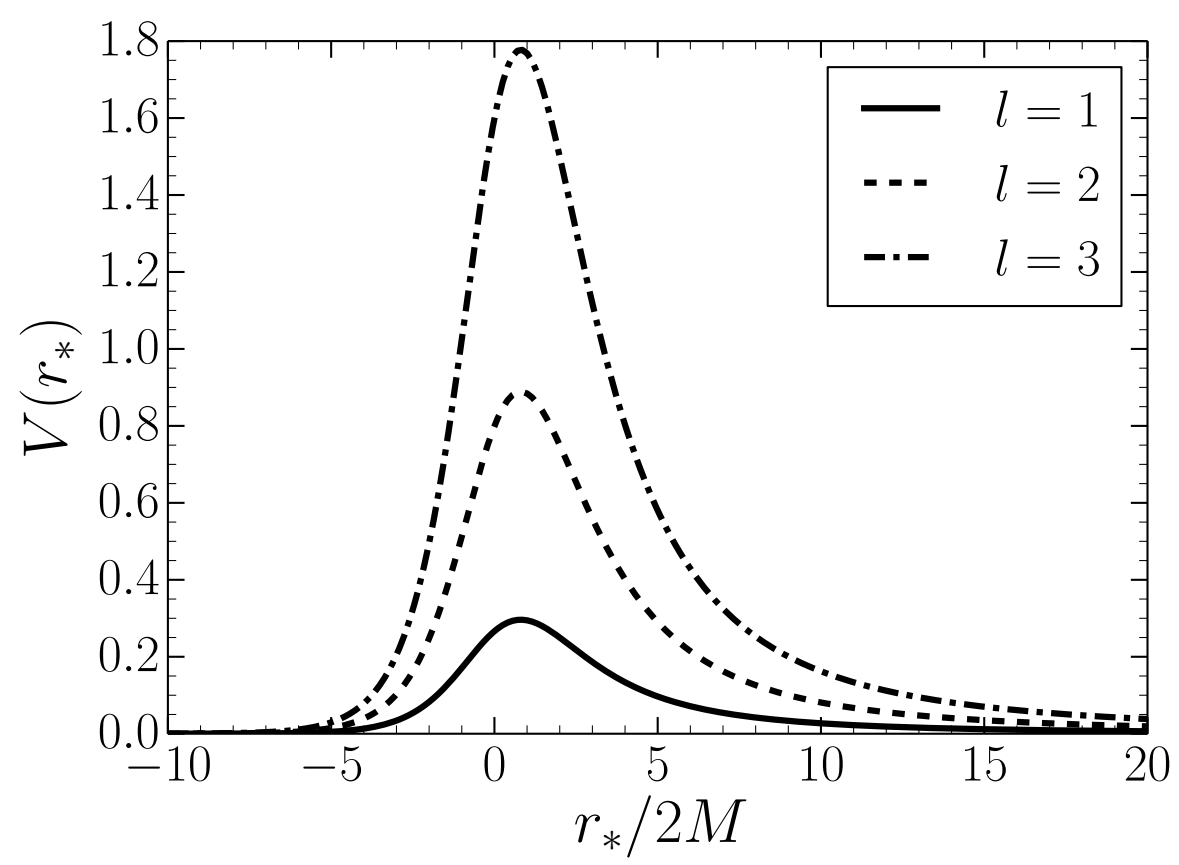

Figure 5.2: The potential barrier (in units of $(2 M)^{2}$ ) for a photon scattered by a Schwarzschild black hole, for increasing angular momentum $\ell$. 


\subsubsection{Graviton}

The analysis of graviton scattering begins by considering linear perturbations to the background Schwarzschild metric eq. 22.40] [32,

$$
\tilde{g}_{a b}=g_{a b}+h_{a b} .
$$

Here $h_{a b}$ is a small perturbation representing the free graviton. A somewhat involved calculation for odd parity perturbations, neglecting all terms $O\left(h^{2}\right)$ and higher, leads to a 1D Schrödinger type equation (e.g. see [26, 27]). This is similar to the scalar, that is, eq. (5.14), but with the potential,

$$
V\left(r_{*}\right)=\left[1-\frac{2 M}{r\left(r_{*}\right)}\right]\left[\frac{\ell(\ell+1)}{r\left(r_{*}\right)^{2}}-\frac{6 M}{r\left(r_{*}\right)^{3}}\right]
$$

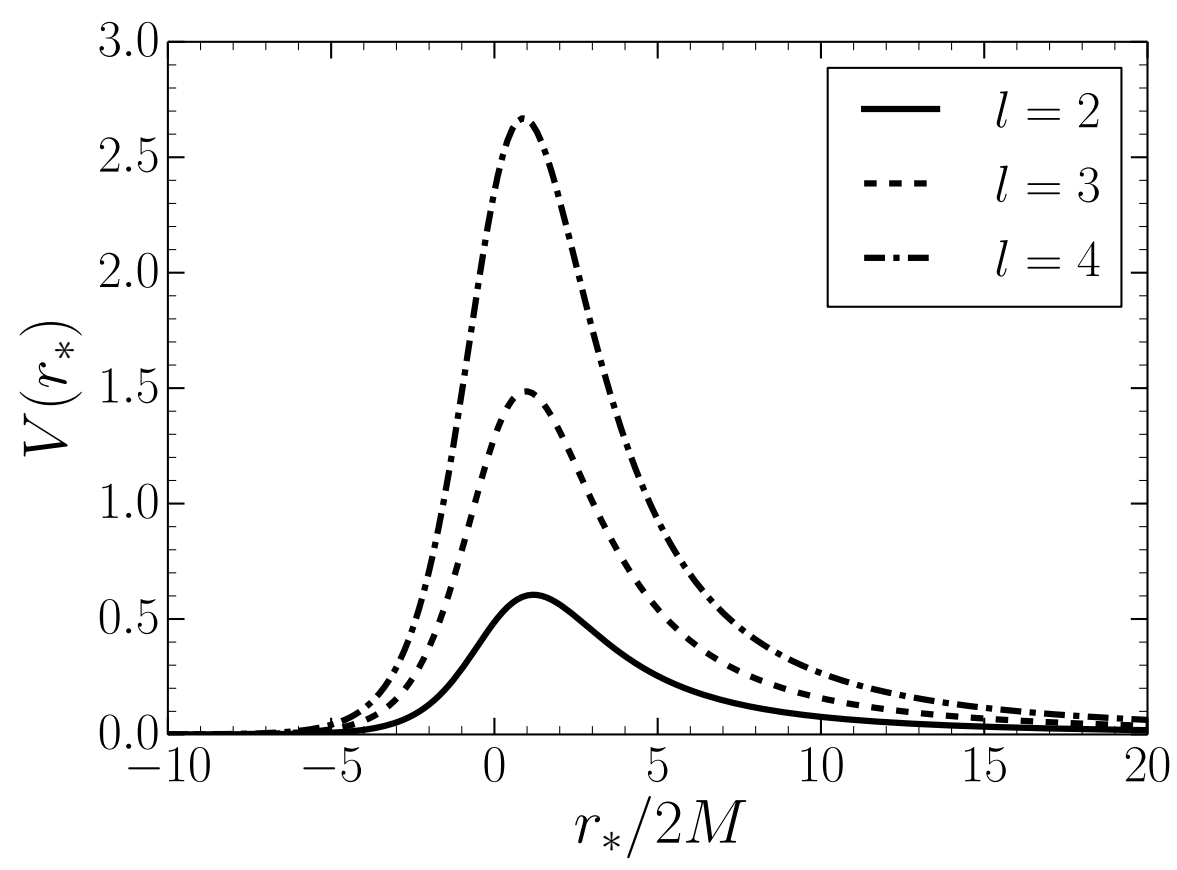

Figure 5.3: The potential barrier (in units of $(2 M)^{2}$ ) for a graviton scattered by a Schwarzschild black hole, for increasing angular momentum $\ell$.

As before, the solution has been decomposed into spherical harmonics where $\ell$ is the principle angular momentum number. Only those solutions with $\ell \geq 2$ are radiative. Fig. (5.3) shows this potential for different $\ell \geq 2$ values. Once again this is very similar to the shape of the scalar but the potential 
has a much higher peak which increases with increasing angular momentum number.

Even parity perturbations leads to a considerably more complicated Zerilli potential [33], however the transmission probabilities from it are the same as those for the Regge-Wheeler potential eq. (5.25] [26]. Thus we need only consider solutions to eq. (5.25) which have the same asymptotic behaviour as for the scalar, that is, eq. (5.18).

\subsubsection{All species}

Summarising these results; the greybody factors associated with scalar, photon, and graviton ( $s=0,1,2$ respectively) emission from black holes are found by expanding the solution in spherical harmonics characterised by the angular momentum number $\ell \geq s$. The problem then reduces to the transmission probability, $T_{\ell, s}(\omega)$, for a solution, $\psi_{\ell}^{(s)}\left(r_{*}\right)$, to a 1D Schrödinger type equation

$$
-\frac{d^{2} \psi_{\ell}^{(s)}}{d r_{*}^{2}}+V_{\ell, s}\left(r_{*}\right) \psi(x)=\omega^{2} \psi_{\ell}^{(s)}\left(r_{*}\right)
$$

with potential,

$$
V_{\ell, s}\left(r_{*}\right)=\left[1-\frac{2 M}{r\left(r_{*}\right)}\right]\left[\frac{\ell(\ell+1)}{r\left(r_{*}\right)^{2}}+\frac{\left(1-s^{2}\right) 2 M}{r\left(r_{*}\right)^{3}}\right] .
$$

where $\psi_{\ell}^{(s)}\left(r_{*}\right)$ satisfies the plane wave asymptotic behaviour

$$
\psi_{\ell}^{(s)}\left(r_{*}\right) \sim\left\{\begin{array}{ll}
e^{-i \omega r_{*}} & r_{*} \rightarrow-\infty \\
A_{\mathrm{out}}^{(\ell, s)} e^{i \omega r_{*}}+A_{\mathrm{in}}^{(\ell, s)} e^{-i \omega r_{*}} & r_{*} \rightarrow+\infty
\end{array} .\right.
$$

The greybody factors for each $\ell, s$, are then

$$
T_{\ell, s}(\omega)=1-\left|\frac{A_{\mathrm{out}}^{(\ell, s)}}{A_{\mathrm{in}}^{(\ell, s)}}\right|^{2}
$$




\subsubsection{Conventional Analysis}

So far, we have developed the formalism for the scattering problem for Schwarzschild black holes, but have yet to solve for the transmission probabilities. To do this for an ordinary scattering problem one first checks to see if there is an analytic solution and then analyse the asymptotic forms to find $A_{\text {in }}$ and $A_{\text {out }}$. It turns out that there is an analytic solution to the ReggeWheeler equation eq. (5.27) in terms of Heun functions [34, 29]. However, this is not particularly useful as not enough is known about the asymptotic forms of the Heun functions.

Where there is no (useful) analytic solution one turns to numerics. In the 1970s Teukolsky and Page [23, 35, 36, 37] numerically solved a slightly different differential equation underlying the scattering/emission process. They used the Teukolsky equation [37] which is equivalent to the Regge-Wheeler potential for a Schwarzschild black hole [26], and thus obtained the transmission coefficients. More recently Phillipp and Perlick [29] use series representations of the Heun functions to numerically calculate the greybody factors. In the next section we shall develop a scheme for numerical approximation based on a transfer matrix approach which is applicable to a wide variety of scattering processes.

In the low frequency limit $M \omega \rightarrow 0$ more is known. Page and Starobinsky [23, 38. have derived the approximate expression,

$$
T_{\ell, s}(\omega) \approx\left[\frac{2^{2 \ell+1}(\ell-s) ! \ell !(\ell+s) !}{(2 \ell) !(2 \ell+1) !}\right]^{2}(M \omega)^{2 \ell+2} .
$$

This is obtained by noting that in the small frequency limit the solutions to the Teukolsky equation [37] become hypergeometric functions. Unfortunately eq. (5.30) is of no use in the intermediate regime $0 \ll M \omega \lesssim 1$, which turns out to be the region of most physical interest; here numerics are required.

\subsection{Transfer Matrix Approach}

We now present the development of analysis a new method based on the transfer matrix. We first present the formalism, then relate the resulting 
path ordered exponential to the product calculus formalism and use this to form the basis for a numerical approximation scheme.

\subsubsection{Transfer Matrix Formalism}

We are looking at calculating the transmission and reflection coefficients for 1D Schrödinger type equations

$$
-\frac{d^{2} \psi}{d x^{2}}+V(x) \psi(x)=E \psi(x)
$$

where the potential asymptotes to a constant,

$$
\lim _{x \rightarrow \pm \infty} V(x)=V_{ \pm \infty}
$$

Later on we will assume that $V_{+\infty}=V_{-\infty}$ but in principle this is not required. In the two asymptotic regions there are two independent solutions [39]

$$
\psi_{ \pm \infty}^{ \pm i}(x) \approx \frac{\exp \left( \pm i \omega_{ \pm \infty}\right)}{\sqrt{\omega_{ \pm \infty}}}
$$

The $\pm i$ refers to right (left) moving modes, $e^{i \omega_{ \pm \infty} x}\left(e^{-i \omega_{ \pm \infty} x}\right)$, and $\omega_{ \pm \infty}=$ $\sqrt{\left(E-V_{ \pm \infty}\right)}$. The two \pm symbols are otherwise unrelated. To analyse the transmission and reflection coefficients we consider Jost solutions, $J_{ \pm}(x)$, which are exact solutions to eq. (5.31) satisfying

$$
J_{ \pm}(x \rightarrow \pm \infty) \rightarrow \frac{\exp \left( \pm i \omega_{ \pm \infty} x\right)}{\sqrt{\omega_{ \pm \infty}}}
$$

and

$$
\begin{aligned}
& J_{+}(x \rightarrow-\infty) \rightarrow \alpha \frac{\exp \left(+i \omega_{-\infty} x\right)}{\sqrt{\omega_{-\infty}}}+\beta \frac{\exp \left(-i \omega_{-\infty} x\right)}{\sqrt{\omega_{-\infty}}} \\
& J_{-}(x \rightarrow+\infty) \rightarrow \alpha^{*} \frac{\exp \left(-i \omega_{+\infty} x\right)}{\sqrt{\omega_{+\infty}}}+\beta^{*} \frac{\exp \left(+i \omega_{+\infty} x\right)}{\sqrt{\omega_{+\infty}}}
\end{aligned}
$$

Here $\alpha$ and $\beta$ are the Bogoliubov coefficients which are related to the reflection and transmission coefficients by,

$$
r=\frac{\beta}{\alpha}, \quad t=\frac{1}{\alpha}
$$


This idea is very similar to the asymptotic behaviour presented before however we avoid the use of $A_{\text {in }}$ and $A_{\text {out }}$ to denote the coefficients of the asymptotic forms as this is a more general context than scattering and radiation by black holes. These solutions are for incoming/right moving waves which are partially scattered and transmitted by the potential $V(x)$. The reflection and transmission probabilities are then given by

$$
R=|r|^{2}, \quad T=|t|^{2} .
$$

That is the probability for an incident particle to be reflected off or transmitted through the potential $V(x)$ is given by $R$ or $T$ respectively. N.b. by definition, the sum of the probabilities for a particle to be reflected and transmitted must be unity,

$$
R+T=1 \quad \Longleftrightarrow \quad|\alpha|^{2}-|\beta|^{2}=1
$$

Now the second order Schrödinger equation (5.31) can be written as a ShabatZakharov system of coupled first order differential equations [40]. To do this write the wave function as

$$
\psi(x)=a(x) \frac{\exp (+i \varphi)}{\sqrt{\varphi^{\prime}}}+b(x) \frac{\exp (-i \varphi)}{\sqrt{\varphi^{\prime}}}
$$

where $a(x)$ and $b(x)$ are arbitrary functions, and the auxiliary function, $\varphi(x)$, is chosen such that it has a non zero derivative, $\varphi^{\prime}(x)$, and

$$
\varphi^{\prime}(x) \rightarrow \omega_{ \pm \infty} \quad \text { as } \quad x \rightarrow \pm \infty
$$

To remove the number of degrees of freedom we can impose the gauge condition,

$$
\frac{d}{d x}\left(\frac{a}{\sqrt{\varphi^{\prime}}}\right) e^{+i \varphi}+\frac{d}{d x}\left(\frac{b}{\sqrt{\varphi^{\prime}}}\right) e^{-i \varphi}=0 .
$$

We now define $\omega(x)^{2} \equiv E-V(x)$ and $\rho \equiv \varphi^{\prime \prime}+i\left[\omega^{2}(x)-\left(\varphi^{\prime}\right)^{2}\right]$, and substitute eq. (5.40) into eq. (5.31) and then use the gauge condition, eq. 5.42, to obtain the following system of equations,

$$
\frac{d}{d x}\left[\begin{array}{l}
a(x) \\
b(x)
\end{array}\right]=\frac{1}{2 \varphi^{\prime}}\left[\begin{array}{cc}
i \operatorname{Im}[\rho] & \rho \exp (-2 i \varphi) \\
\rho^{*} \exp (2 i \varphi) & -i \operatorname{Im}[\rho]
\end{array}\right]\left[\begin{array}{l}
a(x) \\
b(x)
\end{array}\right]
$$


This has the formal solution [39],

$$
\left[\begin{array}{l}
a\left(x_{f}\right) \\
b\left(x_{f}\right)
\end{array}\right]=E\left(x_{0}, x_{f}\right)\left[\begin{array}{l}
a\left(x_{0}\right) \\
b\left(x_{0}\right)
\end{array}\right]
$$

in terms of a generalised position-dependent transfer matrix,

$$
E\left(x_{0}, x_{f}\right)=\mathcal{P} \exp \left(\int_{x_{0}}^{x_{f}} \frac{1}{2 \varphi^{\prime}}\left[\begin{array}{cc}
i \operatorname{Im}[\rho] & \rho \exp (-2 i \varphi) \\
\rho^{*} \exp (2 i \varphi) & -i \operatorname{Im}[\rho]
\end{array}\right] d x\right)
$$

where $\mathcal{P} \exp$ denotes a path ordered exponential operation. Now in the limit $x_{0} \rightarrow-\infty, x_{f} \rightarrow+\infty$ this becomes an exact expression for the Bogoliubov coefficients

$$
\begin{aligned}
{\left[\begin{array}{ll}
\alpha & \beta^{*} \\
\beta & \alpha^{*}
\end{array}\right] } & =E(\infty,-\infty) \\
& =\mathcal{P} \exp \left(\int_{-\infty}^{+\infty} \frac{1}{2 \varphi^{\prime}}\left[\begin{array}{cc}
i \operatorname{Im}[\rho] & \rho \exp (-2 i \varphi) \\
\rho^{*} \exp (2 i \varphi) & -i \operatorname{Im}[\rho]
\end{array}\right] d x\right)
\end{aligned}
$$

In the case that $V_{-\infty}=V_{+\infty}$ there is a natural choice for the auxiliary function, $\varphi(x) \equiv \omega x$ (for simplicity we have written $\omega \equiv \omega_{ \pm \infty}$ ). With this choice eq. 5.45 can be seen to reduce to

$$
E\left(x_{0}, x_{f}\right)=\mathcal{P} \exp \left(-\frac{i}{2 \omega} \int_{x_{0}}^{x_{f}}\left(V(x)-V_{\infty}\right)\left[\begin{array}{cc}
1 & e^{-2 i \omega x} \\
-e^{2 i \omega x} & -1
\end{array}\right] d x\right)
$$

These formal expressions (5.46, 5.47) are very useful for placing bounds on transmission and reflection probabilities in general [39] and for greybody factors for black holes [41, 42]. However we employ them now as a method for direct numerical computation. 


\subsubsection{Path Ordered Exponentials and the Product Cal- culus}

Recall the definition of the path ordered exponential for $A(x)$ a real valued $m \times m$ matrix function:

$$
\mathcal{P} \exp \left(\int_{x_{0}}^{x_{f}} A(x) d x\right) \equiv \lim _{N \rightarrow \infty} \prod_{k=0}^{N-1} \exp \left(A\left(x_{k}^{*}\right) \Delta x_{k}\right)
$$

where $x_{k}^{*} \in\left[x_{k}, x_{k+1}\right]$ is a tag of some partition $\left\{x_{k}\right\}_{k=1}^{N}$ of $\left[x_{0}, x_{f}\right], \Delta x_{k}=$ $\left(x_{k+1}-x_{k}\right)$, and the mesh, $D=\max \left\{\Delta x_{k}\right\}$, goes to zero in the limit $N \rightarrow \infty$. This definition is very much like that of the Riemann integral, and in fact, the path ordered exponential is equivalent to the definition of the product integral [43, 44, 45, 46, 47]. The product calculus is exactly the idea of defining a different notion of calculus based on infinitesimal products and divisions (recall that ordinary calculus is based on infinitesimal sums and subtractions). In the language of product calculus eq. 5.48 becomes

$$
\mathcal{P} \exp \left(\int_{x_{0}}^{x_{f}} A(x) d x\right)=\prod_{x_{0}}^{x_{f}}(\mathcal{I}+A(x) d x)
$$

where the product integral is defined as [45, 46, 47]

$$
\prod_{x_{0}}^{x_{f}}(\mathcal{I}+A(x) d x) \equiv \lim _{N \rightarrow \infty} \prod_{k=0}^{N-1}\left(\mathcal{I}+A\left(x_{k}^{*}\right) \Delta x_{k}\right)
$$

with the partition $\left\{x_{k}\right\}_{k=1}^{N}$ of $\left[x_{0}, x_{f}\right]$, the tag $x_{k}^{*}, \Delta x_{k}$, and the mesh, $D$, defined as before. The equivalence of definitions (5.48, 5.50) can be deduced by noting that all the second order or higher times in $\exp \left(A\left(x_{k}^{*}\right) \Delta x_{k}\right)$ go to zero in the limit $N \rightarrow \infty$ [43]. (Further if the matrix $A(x)$ happens to be nilpotent, i.e. $A(x)^{2}=0$, then the result $\exp \left(A\left(x_{k}^{*}\right) \Delta x_{k}\right)=\mathcal{I}+\left(A\left(x_{k}^{*}\right)\right)$ is exact even be for the limit $D \rightarrow 0$ is taken.) See for instance references [43, 45] for an overview of the product calculus. The following results for the product integral [43, 45, 47] should be noted especially :

- If $\prod_{a}^{b}(\mathcal{I}+A d x)$ exists then $\mathrm{A}$ is bounded on $[a, b]$.

- if $\prod_{a}^{b}(\mathcal{I}+A d x)$ exists and $a \leq u<v \leq b$, then $\prod_{u}^{v}(I+A d x)$ exists . 
- if $a<b<c$, and $\prod_{a}^{b}(\mathcal{I}+A d x)$ and $\prod_{b}^{c}(\mathcal{I}+A d x)$ exist then,

$$
\prod_{a}^{c}(\mathcal{I}+A d x)=\prod_{a}^{b}(\mathcal{I}+A d x) \cdot \prod_{b}^{c}(\mathcal{I}+A d x)
$$

- $\prod_{a}^{b}(\mathcal{I}+A d x)$ exists, if and only if, $\int_{a}^{b} A d x$ exists

- if $\prod_{a}^{b}(\mathcal{I}+A d x)$ exists then,

$$
\begin{aligned}
\prod_{a}^{b}(I+A d x)= & \mathcal{I}+\int_{a}^{b} A\left(x_{1}\right) d x_{1}+\int_{a}^{b} \int_{a}^{x_{1}} A\left(x_{1}\right) A\left(x_{2}\right) d x_{2} d x_{1} \\
& +\int_{a}^{b} \int_{a}^{x_{1}} \int_{a}^{x_{2}} A\left(x_{1}\right) A\left(x_{2}\right) A\left(x_{3}\right) d x_{3} d x_{2} d x_{1}+\ldots
\end{aligned}
$$

Eq. 5.52 is commonly known in the mathematics community as the Peano series and as the Dyson series in the physics community. It is the physics community's standard method for approximating path ordered exponentials and can offer some more analytic insight as it yields a successively better analytic approximation to the Bogoliubov coefficients. Using an ordering operator $\mathcal{P}$, as in chapter 3 , eq. 5.52 can be written,

$$
\prod_{a}^{b}(I+A d x)=\sum_{n=0}^{\infty} \frac{1}{n !} \int_{a}^{b} d x_{1} \int_{a}^{b} d x_{2} \ldots \int_{a}^{b} d x_{n} \mathcal{P}\left\{A\left(x_{1}\right) A\left(x_{2}\right) \ldots A\left(x_{n}\right)\right\}
$$

where,

$$
\mathcal{P}\left\{A\left(x_{1}\right) A\left(x_{2}\right) \ldots A\left(x_{n}\right)\right\}=A\left(x_{\sigma(1)}\right) A\left(x_{\sigma(2)}\right) . . A\left(x_{\sigma(n)}\right) .
$$

with $\sigma_{(i)}$ any permutation such that $x_{\sigma(1)} \geq x_{\sigma(2)} \geq \ldots \geq x_{\sigma(n)}$. For example,

$$
\mathcal{P}\left\{A\left(x_{1}\right) A\left(x_{2}\right)\right\}=\Theta\left(x_{1}-x_{2}\right) A\left(x_{1}\right) A\left(x_{2}\right)+\Theta\left(x_{2}-x_{1}\right) A\left(x_{2}\right) A\left(x_{1}\right)
$$

where $\Theta(x)$ is again the Heaviside step function. 


\subsubsection{Example: a Delta Function Potential}

As a first example we apply this formalism to a Dirac-Delta function, $\delta(x)$, potential of the form

$$
V_{\text {Delta }}=V_{0} \delta(x)
$$

This is a situation in which the transmission and reflection probabilities can be calculated explicitly by analysing the incoming and outgoing plane wave solutions to eq. (5.31) with potential eq. (5.56). The analytic expression for transmission probability, with our notation, is, in this example,

$$
T=\frac{1}{1+\frac{V_{0}^{2}}{4 \omega^{2}}}
$$

Now in the transfer matrix formalism the Bogoliubov coefficients becomes

$$
\left[\begin{array}{ll}
\alpha & \beta^{*} \\
\beta & \alpha^{*}
\end{array}\right]=\prod_{-\infty}^{+\infty}\left(\mathcal{I}+\delta(x) A_{\mathrm{D}}(x) d x\right)
$$

where

$$
A_{\mathrm{D}}(x)=-\frac{i V_{0}}{2 \omega}\left[\begin{array}{cc}
1 & e^{-2 i \omega x} \\
-e^{2 i \omega x} & -1
\end{array}\right] .
$$

Now using eq. (5.54) this can be written,

$$
\begin{aligned}
{\left[\begin{array}{cc}
\alpha & \beta^{*} \\
\beta & \alpha^{*}
\end{array}\right] } & =\sum_{n=0}^{\infty} \frac{1}{n !} \int_{-\infty}^{+\infty} d x_{1} \int_{-\infty}^{+\infty} d x_{2} \ldots \int_{-\infty}^{+\infty} d x_{n} \delta\left(x_{1}\right) \delta\left(x_{2}\right) \ldots \delta\left(x_{n}\right) \\
& \times \mathcal{P}\left\{A_{\mathrm{D}}\left(x_{1}\right) A_{\mathrm{D}}\left(x_{2}\right) \ldots A_{\mathrm{D}}\left(x_{n}\right)\right\} \\
& =\sum_{n=0}^{\infty} \frac{\left(A_{\mathrm{D}}(0)\right)^{n}}{n !}=\exp \left(A_{\mathrm{D}}(0)\right)
\end{aligned}
$$

Finally noting $A_{\mathrm{D}}(x)^{2}=0$, this becomes

$$
\left[\begin{array}{ll}
\alpha & \beta^{*} \\
\beta & \alpha^{*}
\end{array}\right]=\left[\begin{array}{cc}
1-\frac{i V_{0}}{2 \omega} & -\frac{i V_{0}}{2 \omega} \\
+\frac{i V_{0}}{2 \omega} & 1+\frac{i V_{0}}{2 \omega}
\end{array}\right],
$$

which using eq. (5.37) and eq. (5.38) can be seen to agree with the result eq. (5.57).

Having shown the success of the method in the next section we show how 
to turn this method into an accurate numerical approximation scheme for situations in which there is no known analytic expression for the transmission probabilities. In particular we present the calculation of the greybody factors for the Schwarzschild black hole and evaluate some of the important physical quantities.

\subsection{Numerical Results}

We use the Product Calculus formalism as a basis for numerical calculation of the greybody factors. In the form of eq. (5.49) the transfer matrix is particularly simply to evaluate numerically. Defining

$$
A\left(x_{k}\right) \equiv-\frac{i}{2 \omega} V\left(x_{k}\right)\left[\begin{array}{cc}
1 & e^{-2 i \omega x_{k}} \\
-e^{2 i \omega x_{k}} & -1
\end{array}\right]
$$

the transfer matrix can be compactly written,

$$
\begin{aligned}
E\left(x_{0}, x_{f}\right) & =\lim _{N \rightarrow \infty} \prod_{k=1}^{N}\left(\mathcal{I}+A\left(x_{k}\right) h\right) \\
& =\lim _{N \rightarrow \infty}\left\{\left(\mathcal{I}+A\left(x_{N-1}\right) h\right) \ldots\left(\mathcal{I}+A\left(x_{2}\right) h\right)\left(\mathcal{I}+A\left(x_{1}\right) h\right)\right\} .
\end{aligned}
$$

Where $h=\left(x_{f}-x_{0}\right) / N$, and $x_{k}=x_{0}+k h$. This expression is extremely easy to compute numerically, however, much like a simple Riemann sum, convergence is rather slow. In order to improve convergence, Helton and Stuckwisch [47] introduce a polynomial approximation which has error bounded by $H\left(x_{0}, x_{f}\right) h^{p}$. Here, $H\left(x_{0}, x_{f}\right)$ is a bounded interval function determined by the matrix $A$, and $p$ is the order of the polynomial approximation. This is essentially the equivalent of a higher order Simpson type approximation for the product integral. There is a small technicality that Helton and Stuckwisch use a definition of the product integral based on right hand multiplication, i.e. the order of the products in eq. (5.50) is reversed. This can be related 
to the form in eq. (5.50) by noting in the limit $N \rightarrow \infty$,

$$
\begin{aligned}
{\left[\prod_{x_{0}}^{x_{f}}(\mathcal{I}+\right.} & A(x) d x)]^{-1} \\
& =\left[\lim _{N \rightarrow \infty}\left\{\left(\mathcal{I}+A\left(x_{N-1}\right) h\right) \ldots\left(\mathcal{I}+A\left(x_{2}\right) h\right)\left(\mathcal{I}+A\left(x_{1}\right) h\right)\right\}\right]^{-1} \\
& =\lim _{N \rightarrow \infty}\left\{\left[\left(\mathcal{I}+A\left(x_{N-1}\right) h\right) \ldots\left(\mathcal{I}+A\left(x_{2}\right) h\right)\left(\mathcal{I}+A\left(x_{1}\right) h\right)\right]^{-1}\right\} \\
& =\lim _{N \rightarrow \infty}\left\{\left(\mathcal{I}-A\left(x_{1}\right) h\right)\left(\mathcal{I}-A\left(x_{2}\right) h\right) \ldots\left(\mathcal{I}-A\left(x_{N-1}\right) h\right)\right\},
\end{aligned}
$$

Thus by sending $A\left(x_{i}\right) \rightarrow-A\left(x_{i}\right)$ and using the approximation in [47] we obtain the inverse of the product integral we set out to calculate. Since the Bogoliubov matrix satisfies

$$
\left[\begin{array}{ll}
\alpha & \beta^{*} \\
\beta & \alpha^{*}
\end{array}\right]^{-1}=\left[\begin{array}{cc}
\alpha^{*} & -\beta \\
-\beta^{*} & \alpha
\end{array}\right],
$$

we see that there is no loss of information; this method lends itself very well to finding the transmission probabilities.

The 5th order approximation Helton and Stuckwisch introduce is described in [47],

$$
\begin{aligned}
& \prod_{k=1}^{N}\left[\mathcal{I}+(h / 90)\left(7 A_{0}+32 A_{1}+12 A_{2}+32 A_{3}+7 A_{4}\right)\right. \\
& +\left(h^{2} / 90\right)\left(8 A_{0} A_{1}-12 A_{0} A_{2}+18 A_{0} A_{3}-7 A_{0} A_{4}+18 A_{1} A_{2}-12 A_{1} A_{3}\right. \\
& \left.\quad+18 A_{1} A_{4}+18 A_{2} A_{3}-12 A_{2} A_{4}+8 A_{3} A_{4}\right) \\
& \quad+\left(h^{3} / 60\right)\left(3 A_{0} A_{1} A_{2}-2 A_{0} A_{1} A_{3}+3 A_{0} A_{1} A_{4}-2 A_{0} A_{2} A_{3}-2 A_{0} A_{2} A_{4}\right. \\
& \left.\quad+3 A_{0} A_{3} A_{4}+8 A_{1} A_{2} A_{3}-2 A_{1} A_{2} A_{4}-2 A_{1} A_{3} A_{4}+3 A_{2} A_{3} A_{4}\right) \\
& \quad+\left(h^{4} / 120\right)\left(4 A_{0} A_{1} A_{2} A_{3}-A_{0} A_{1} A_{2} A_{4}-A_{0} A_{1} A_{3} A_{4}\right. \\
& \left.\quad-A_{0} A_{2} A_{3} A_{4}+4 a_{1} A_{2} A_{3} a_{4}\right) \\
& \left.\quad+\left(h^{5} / 120\right) A_{0} A_{1} A_{2} A_{3} A_{4} A_{5}\right],
\end{aligned}
$$


where $A_{j}=A\left[x_{k-1}+j h / 4\right]$ for $j=0,1,2,3,4$ and $k=1,2, . ., N$. This rather unwieldy expression they show can compactly be written as,

$$
\prod_{k=1}^{N}\left[\mathcal{I}+\left(28 K_{1}+32 K_{2}+6 K_{3}+4 K_{4}+K_{5}\right) / 360\right]
$$

where

$$
\begin{aligned}
& K_{1}=h A_{4} \\
& K_{2}=h A_{3}\left(4 \mathcal{I}+K_{1}\right) \\
& K_{3}=h A_{2}\left[8\left(\mathcal{I}-K_{1}\right)+3 K_{2}\right] \\
& K_{4}=h A_{1}\left[32 \mathcal{I}+18 K_{1}+3 h A_{2}\left(6 \mathcal{I}-K_{1}+K_{2}\right)-3 K_{2}\right] \\
& K_{5}=h A_{0}\left[28\left(\mathcal{I}-K_{1}\right)-3 h A_{2}\left(16 \mathcal{I}+4 K_{1}+K_{2}\right)+K_{4}+18 K_{2}\right] .
\end{aligned}
$$

This scheme is our primary tool for actually calculating the Transmission probabilities.

\subsubsection{Example: a Square Barrier Potential}

As a first example of numerically evaluating transmission and reflection probabilities via the product calculus we present the simplest situation of a square barrier. That is, the potential is of the form,

$$
V(x)=V_{0} \Theta(a-|x|)
$$

Thus, the Bogoliubov coefficients can be written,

$$
\begin{aligned}
{\left[\begin{array}{ll}
\alpha & \beta^{*} \\
\beta & \alpha^{*}
\end{array}\right] } & =\mathcal{P} \exp \left(-\frac{i V_{0}}{2 \omega} \int_{-\infty}^{+\infty} \Theta(a-|x|)\left[\begin{array}{cc}
1 & e^{-2 i \omega x} \\
-e^{2 i \omega x} & -1
\end{array}\right] d x\right) \\
& =\mathcal{P} \exp \left(-\frac{i V_{0}}{2 \omega} \int_{-a}^{+a}\left[\begin{array}{cc}
1 & e^{-2 i \omega x} \\
-e^{2 i \omega x} & -1
\end{array}\right] d x\right)
\end{aligned}
$$

Defining,

$$
A_{\mathrm{SB}}(x)=-\frac{i V_{0}}{2 \omega}\left[\begin{array}{cc}
1 & e^{-2 i \omega x} \\
-e^{2 i \omega x} & -1
\end{array}\right]
$$


eq. 5.70 becomes, in the language of product calculus,

$$
\left[\begin{array}{cc}
\alpha & \beta^{*} \\
\beta & \alpha^{*}
\end{array}\right]=\prod_{-a}^{a}\left(\mathcal{I}+A_{\mathrm{SB}}(x) d x\right) .
$$

Now the square barrier case is another of the few situations in which the transmission and reflection probabilities can be calculated explicitly by analysing the incoming and outgoing plane wave solutions to eq. (5.31) with the potential eq. (5.69). In our notation and units the transmission probability is given by,

$$
T= \begin{cases}\frac{1}{1+\frac{V_{0}^{2}}{4} \frac{\sinh ^{2}\left(2 a \sqrt{V_{0}-\omega^{2}}\right)}{\omega^{2}\left(V_{0}-\omega^{2}\right)}} & \omega^{2}<V_{0} \\ \frac{1}{1+\frac{V_{0}^{2}}{4} \frac{\sin ^{2}\left(2 a \sqrt{\omega^{2}-V_{0}}\right)}{\omega^{2}\left(\omega^{2}-V_{0}\right)}} & \omega^{2} \geq V_{0}\end{cases}
$$

and c.f. eq. (5.39) $R=1-T$. These are shown in Fig. 5.4 for the values $(2 a)^{2} V_{0}=1,10$. The numerical calculation of eq. 5.72) was implemented in Python and then compared to the exact expression eq. (5.73). The relative difference between the two is shown in Fig. 5.5
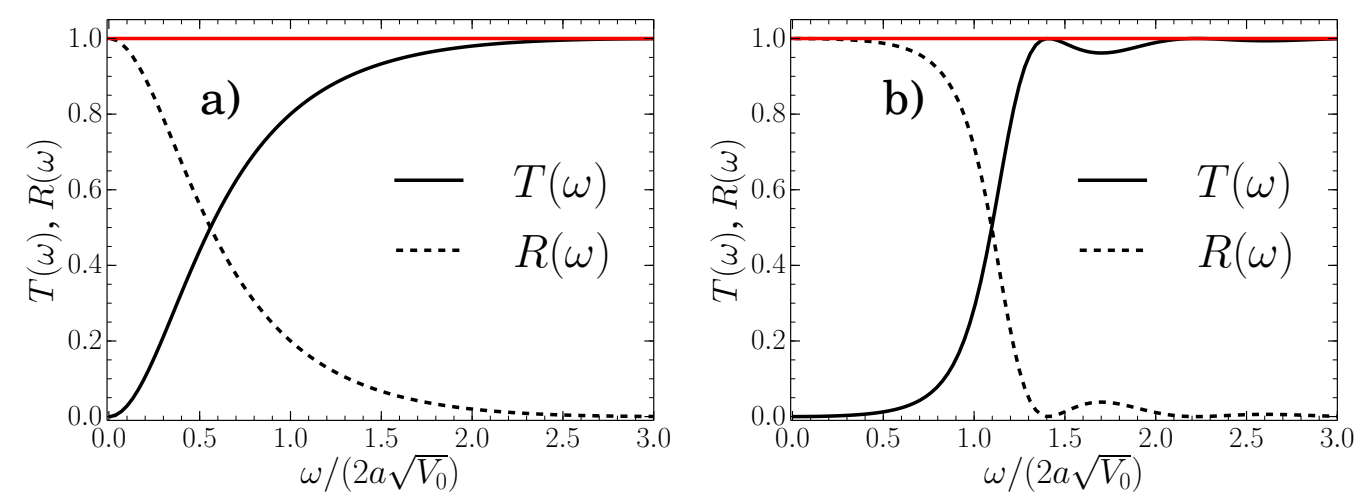

Figure 5.4: The exact expression for the transmission and reflection probabilities of a square barrier 5.73 shown for two different values of $V$ : a) shows $(2 a)^{2} V_{0}=1$, and, b), $(2 a)^{2} V_{0}=10$.

It can be seen in Fig. 5.5 that the product integral calculation very quickly approaches the exact value. With only 4 terms the error is already less than $\sim O\left(10^{-5}\right)$ for $(2 a)^{2} V_{0}=1$, and, in each case by the time 512 terms are used, the approximation is approaching the working tolerance of the double precision used. This is consistent with the error bounds introduced by Helton 
and Stuckwisch [47.
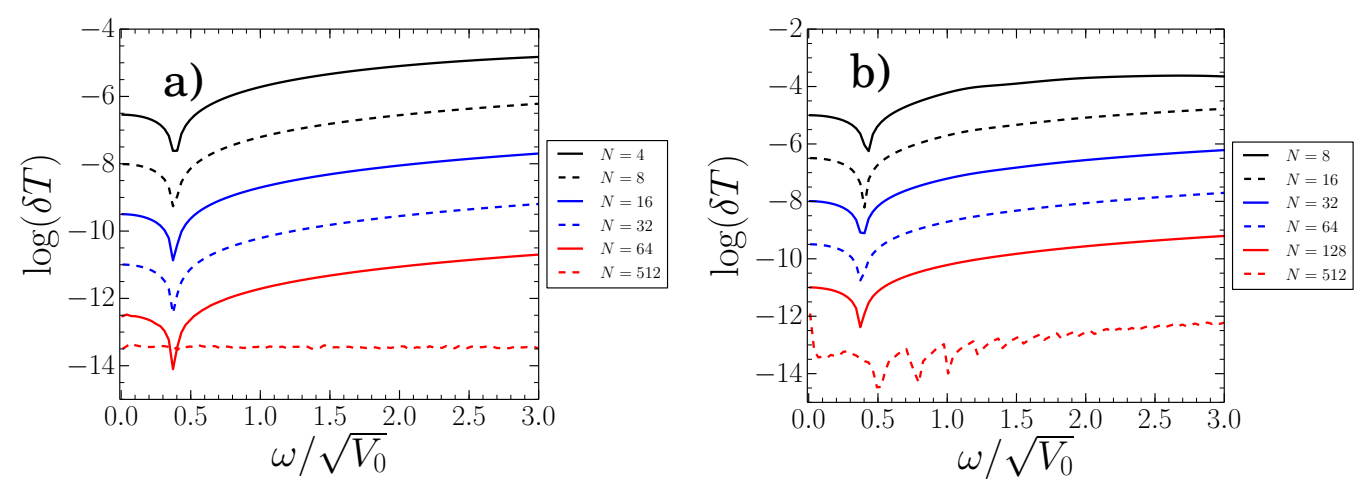

Figure 5.5: The relative difference, $\delta T \equiv\left|T_{\text {Exact }}(\omega)-T_{N}(\omega)\right| / T_{\text {Exact }}$, of the $N$-th approximation to the product integral calculation of the transmission probability to the exact expression, a), shows for $(2 a)^{2} V_{0}=1$, b), for $(2 a)^{2} V_{0}=10$.

\subsubsection{Greybody Factors from a Schwarzschild Black Hole}

Here we present the numerical results for the analysis of the Regge-Wheeler potential eq. (5.27) from which we obtain the Schwarzschild greybody factors. Now recall this potential vanishes in the asymptotic regions (i.e $V\left(r_{*}\right) \rightarrow$ 0 , as $r_{*} \rightarrow \pm \infty$ ), and so using eq. (5.37) and eq. (5.38) the transmission probabilities can be calculated from the Bogoliubov coefficients, eq. (5.46). In this case the transfer matrix becomes

$$
\left[\begin{array}{cc}
\alpha & \beta^{*} \\
\beta & \alpha^{*}
\end{array}\right]=\mathcal{P} \exp \left(-\frac{i}{4 x} \int_{-\infty}^{+\infty} V\left(u_{*}\right)\left[\begin{array}{cc}
1 & \exp \left(-4 i x u_{*}\right) \\
-\exp \left(4 i x u_{*}\right) & -1
\end{array}\right] d u_{*}\right)
$$

Here the potential eq. 5.27

$$
V\left(u_{*}\right)=\left[1-\frac{1}{\left.u\left(u_{*}\right)\right)}\right]\left[\frac{\ell(\ell+1)}{u\left(u_{*}\right)^{2}}+\frac{\left(1-s^{2}\right)}{u\left(u_{*}\right)^{3}}\right],
$$


is written in terms of the dimensionless variables $u_{*}=r_{*} / 2 M$ and $u\left(u_{*}\right)=$ $r\left(u_{*}\right) / 2 M$. In the product calculus formalism this is

$$
\left[\begin{array}{cc}
\alpha & \beta^{*} \\
\beta & \alpha^{*}
\end{array}\right]=\prod_{-\infty}^{+\infty}\left(\mathcal{I}+A\left(u_{*}\right) d u_{*}\right)
$$

where

$$
A\left(u_{*}\right) \equiv-\frac{i}{4 x} V\left(u_{*}\right)\left[\begin{array}{cc}
1 & e^{-4 i x u_{*}} \\
-e^{4 i x u_{*}} & -1
\end{array}\right]
$$
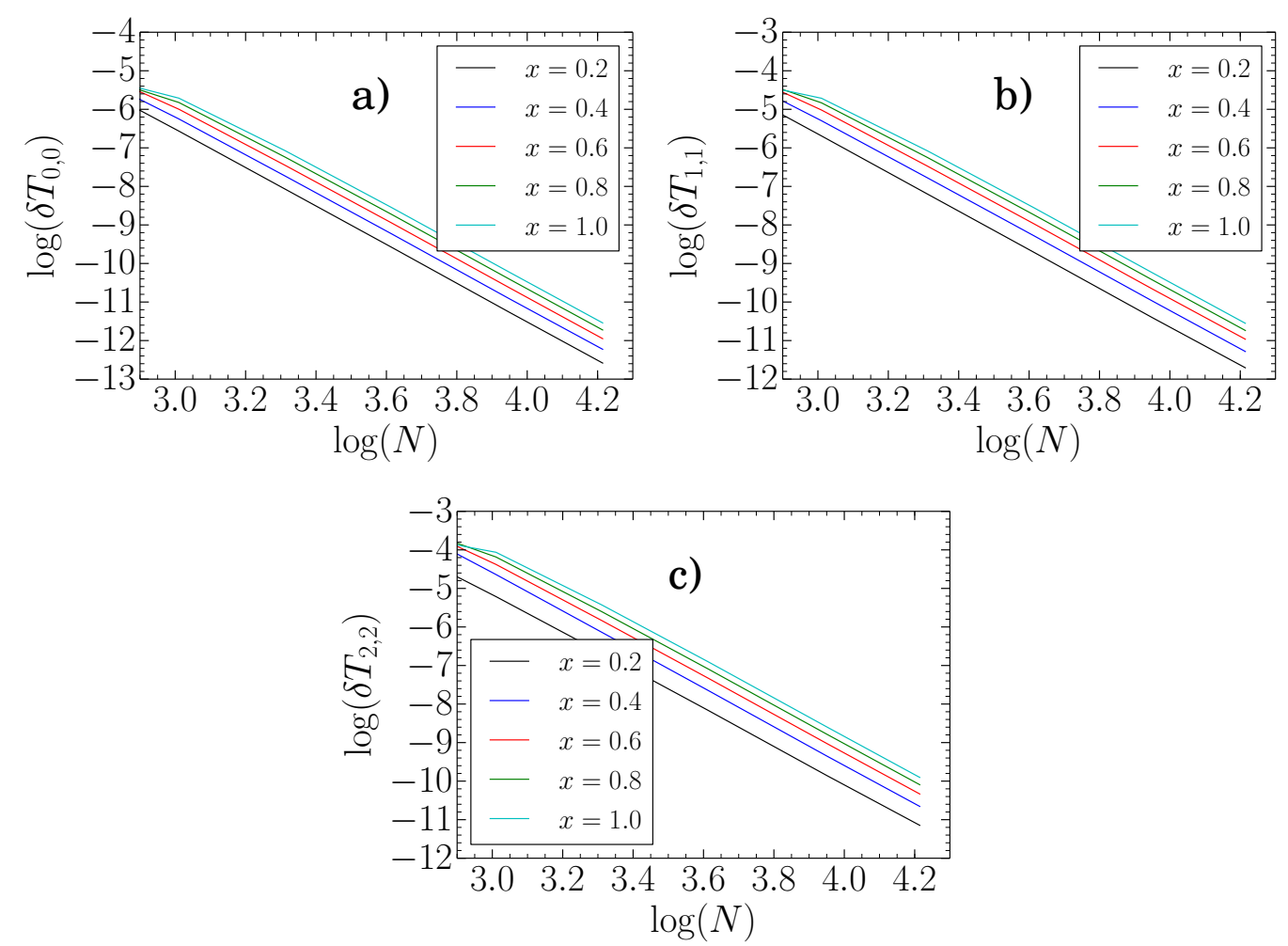

Figure 5.6: Convergence rates of transmission probabilities, $T_{s, s}(x)$, for, (a) scalars, (b) photons, (c) gravitons, where $\delta T_{s, s} \equiv \mid T_{s, s}^{(N)}(x)-$ $T_{s, s}^{(N+1)}(x) \mid / T_{s, s}^{(N)}(x)$ is the relative error of the $N$-th approximation, (i.e. $\mathrm{N}$ terms).

The calculation for the transmission probabilities was numerically implemented in Python using the polynomial approximation, eq. (5.67). The integration region $[-\infty,+\infty]$ was approximated by $[-50,350]$, which was found to introduce an error $\sim O\left(10^{-9}\right)$. The asymmetry of this region stems from the asymmetry in the decay of the potential, see Figs. 5.1, 5.2, and 5.3. The convergence of product integrals themselves is shown in Fig. 5.6. 

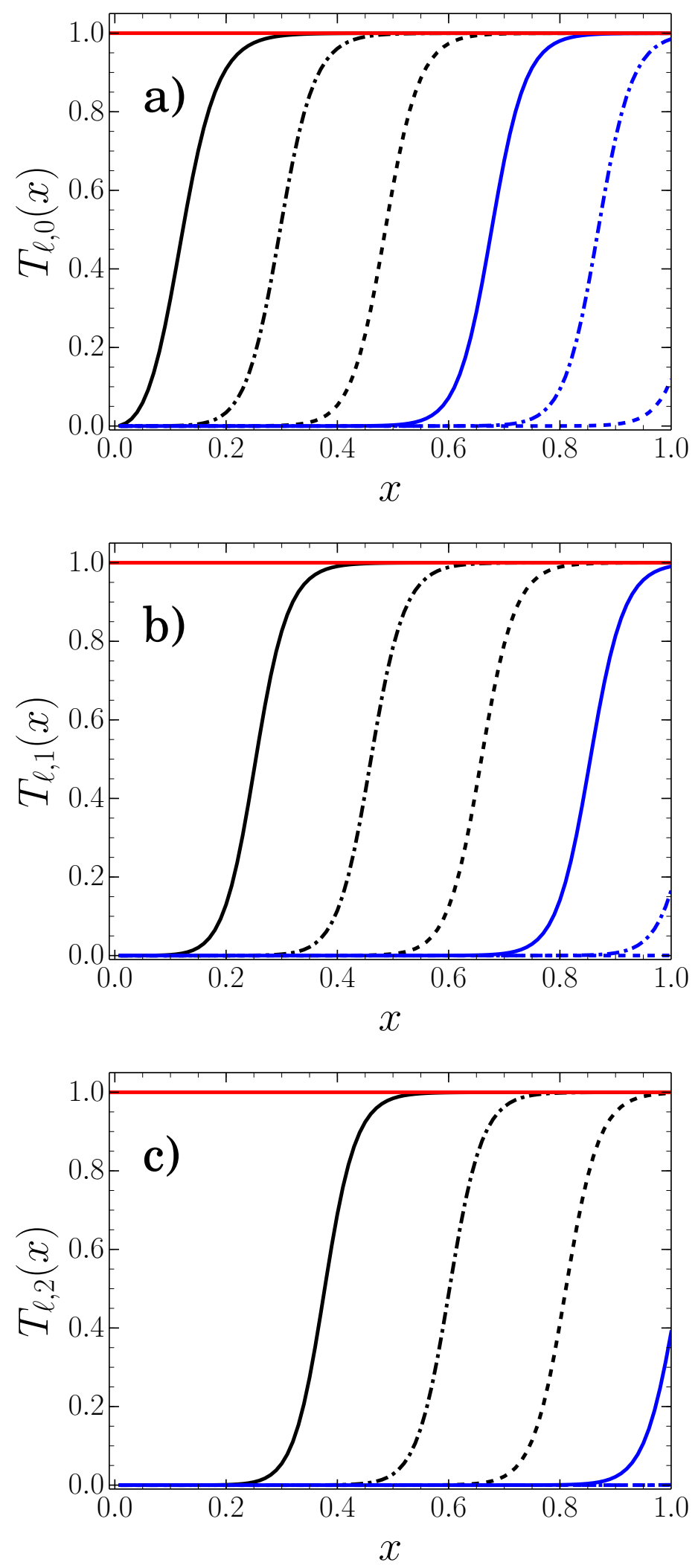

Figure 5.7: Plots of the transmission probabilities, $T_{\ell, s}(x)$, as a function of $x=M \omega$ for, a) scalars, b) photons, and c) gravitons. The left most function on each plot corresponds to the $\ell=s$ transmission probability, increasing $\ell$ values occur to the right. 
Fig. 5.7 shows the transmission probability for each of the scalar, photon, and graviton cases. It can be seen that: the scalar $s=0$ case has transmission at the lowest frequencies, for each species larger $\ell$ values require higher frequencies before there is any transmission through the barrier. Also each species shows that, for every $\ell$ mode, eventually there is complete transmission through the barrier, at high enough frequencies. These remarks can be interpreted physically by observing the potential $V\left(r_{*}\right)$ is lowest for the $\ell=0=s$ case and, as such, less energy (i.e. lower frequency) is required to pass through the barrier. For larger $\ell$ and $s$ values the potential is higher and so more energy is required. Eventually any particle species in any mode will have enough energy to completely pass through the barrier. That is $T_{\ell, s}(x) \rightarrow 1$ as $x \rightarrow \infty$
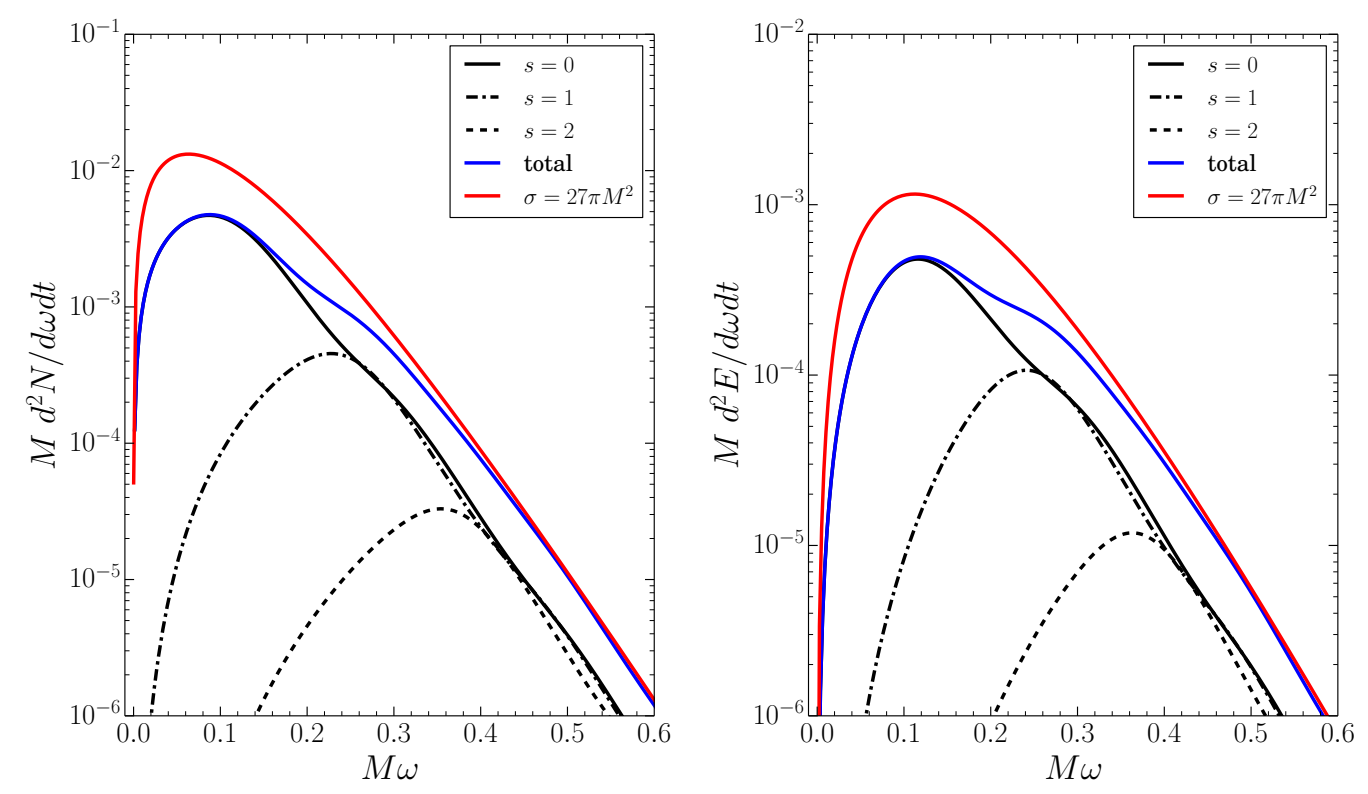

Figure 5.8: Plots of the number (Energy) emission spectrum, left (right), for a Schwarzschild black hole, c.f. eq. (5.1) and eq. (5.3). Note the logarithmic scale. The emission spectrum is dominated by scalar particles, and emission rates decrease with increasing spin. The total emission rates (i.e. summing over all particle species) are bounded by the sums over the geometric optics limit, $\sigma=27 \pi M^{2}$, for each species (shown in red), and this limit is approached as $x=M \omega \rightarrow \infty$.

Fig. 5.8 shows plots of the number and energy emission rates (eq. (5.1) and eq. (5.3)). It can be seen that for $x>0.6$ the emission rate becomes rapidly negligible, exponentially decaying to zero. These figures can be compared to 
the results obtained by Page [23] and are found to be in very precise agreement. The emission of particles is dominated by scalars, and the rate reduces with increasing spin. This can be understood from Fig. 5.7, in which, it can be seen that the transmission probabilities of lower spin become significant more quickly (for smaller $x$ ) which coincides with the peak in the probability spectrum $P_{\ell, s}(x)$. The emission rates cannot be accurately obtained from a black body approximation but this does provide a useful semi-analytic estimate for the process (see work conducted in conjunction with Sebastian Schuster, Matt Visser, and Alexander Van-Brunt [1]). Furthermore approximation schemes to the greybody factors based on low or high energy requirements do not adequately capture the region of most emission $0<x<0.2$ (for details see the results in collaboration with Matt Visser [2]).

Fig. 5.9 shows a plot of the dimensionless cross-section for each species of particle. As $x$ increases the cross-section approaches the geometric optics limit, that is, $S(x) \rightarrow 1$. This happens more quickly for lower spins. Each species also shows oscillations about the classical limit which are due to the transmission probabilities becoming appreciable for increasing $\ell$ values, weighted by the $2 \ell+1$ factor.

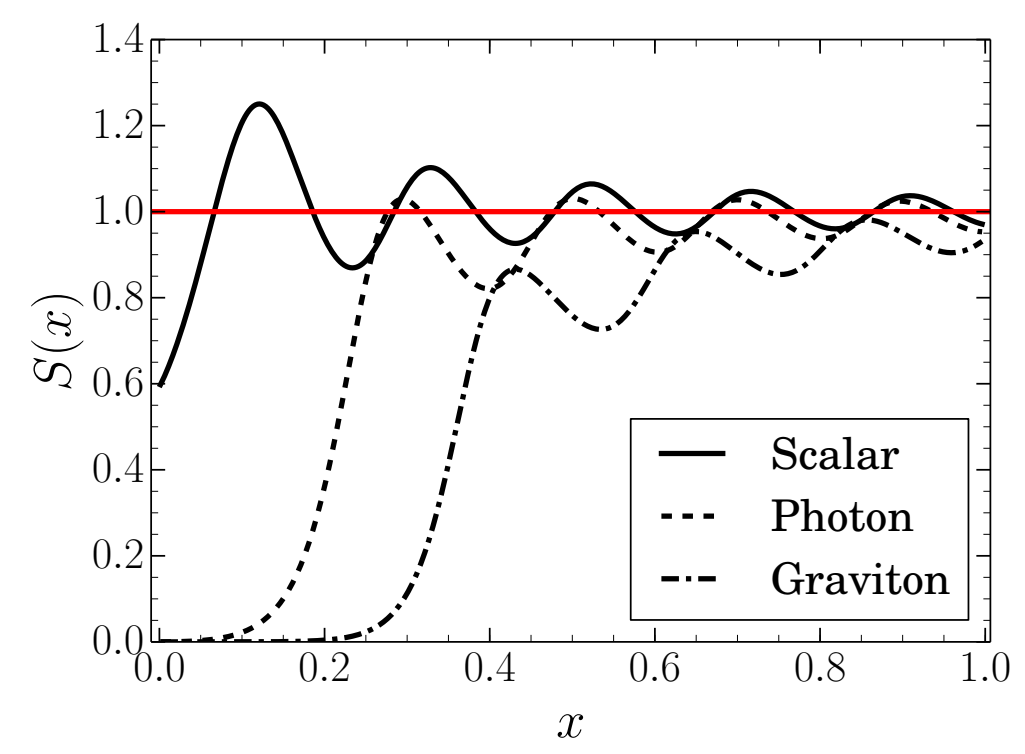

Figure 5.9: The dimensionless cross-section, $S(x)$ (eq. (5.5)) as a function $x=$ $M \omega$. Here it is shown for scalars, photons, and gravitons for a Schwarzschild black hole. 


\subsection{Implications and Extensions}

In this chapter we have developed a new method for numerical calculation of the greybody factors which has wide application to other one dimensional scattering problems. Although we have only applied it to spherically symmetric black holes there is certainly scope to apply the scheme to the more general cases. We now talk briefly about some of the implications the greybody factors have on the emission process and how our method might be extended.

\subsubsection{Implications}

One of the strongest effects of the greybody factors on the Hawking process is that they significantly reduce the emission rate, especially for higher spins, see Fig. 5.8. Fig. 5.8 also shows that a semi-analytic treatment using the geometric optics limit of the cross-section provides an upper bound on the total emission rate. Now the Hawking emission process is already sparse when treated as a semi-analytic black body [1] and the greybody factors only serve to worsen the situation. It may therefore be productive to view the Hawking evaporation in terms of a two-body decay process. Further any symmetry assumptions about the process are only valid when interpreted as statements about long time statistical averages. These comments provide tantalising hints about how to reinterpret the process.

These statements are certainly true for uncharged non-rotating, that is, Schwarzschild black holes. However difficulties arise when looking at ReissnerNordström, Kerr, and Kerr-Newman black holes. When considering these situations one must consider super-radiance wherein the Bose occupation number diverges and changes sign simultaneously with the greybody factors. Page [23, 35, 36] has shown that this can lead to greatly enhanced emission rates, i.e. super-radiance. However there is good reason to separate the super-radiant emission from the Hawking emission [1] which allows us to say generically that the Hawking radiation process is characterised by the long gaps between successive emissions. 


\subsubsection{Extensions}

It turns out that the scattering of particles by rotating and charged black hole can be described by the appropriate Teukolsky equation [23, 35, 36, 37. This is a second order differential equation which can be put into the form of eq. (5.26) with appropriate potential. This means that the transfer matrix method developed in sections three and four of this chapter could be used to find the greybody factors in more general situations. Difficulties would arise from the fact that without spherical symmetry each azimuthal mode must be treated separately and there is no longer an analytic expression for the eigenvalue of the generalised spheroidal harmonics arising from the angular equation from a separation of variables. $($ C.f. $\ell(\ell+1)$ for the Schwarzschild black hole).

Further work may also be done making use of the Dyson/Peano series eq. (5.52) to develop semi-analytic approximation schemes for greybody factors. The difficulty in this approach is evident from the problem of solving iterated integrals both analytically and numerically.

The work in this chapter has been included in two papers; the first (in collaboration with Sebastian Schuster, Matt Visser, and Alexander Van-Brunt) has been recently published in Classical and Quantum Gravity [1] and the second (in collaboration with Matt Visser) is in the submission process and available online [2].

In the next chapter we look at the possible extensions of Wick rotation to curved spacetime motivated by the results in QFT in Minkowski spacetime and the Euclidean derivation of Hawking radiation. 


\section{Chapter 6}

\section{Wick Rotation in Curved Spacetime}

We have seen in Chapter 4 that analytically continuing the time coordinate of the Schwarzschild metric, eq. (2.40), leads to the Hawking temperature [21]. This suggests a possible route to a quantisation of gravity via the Feynman path integral route and the Euclidean techniques presented in Chapter 3. Known as Euclidean Quantum Gravity [48, 49, 50] this viewpoint provides further motivation for a generic way to Wick rotate. Another question to ask is: given a spacetime can we unambiguously define a Wick rotation for the propagators of a quantum field on that spacetime? We will address these issues in this chapter. The first section is devoted to how one might write down a Euclidean quantisation of gravity. In the second we present examples of where a traditional Wick rotation can and cannot be employed. We then review the methods to find a general procedure. Finally we highlight the problems with Wick rotation and present possible future directions.

\subsection{Euclidean Quantum Gravity}

The Feynman path integral approach offers an intuitive way to conceptual a route to a quantised theory of gravity, and its history goes back to the 1950s [51]. Proceeding in a similar manner to Chapter 3, one can consider the probability of going from one metric and field configuration $g_{1}, \phi_{1}$ on a surface $\Sigma_{1}$, to another $g_{2} \phi_{2}, \Sigma_{2}$, to be the sum over all the field configurations 
satisfying the surface values [48, 49, 50,

$$
\left\langle g_{2}, \phi_{2}, \Sigma_{2} \mid g_{1}, \phi_{1}, \Sigma_{1}\right\rangle=\int D[g, \phi] \exp (i S[g, \phi])
$$

Here $D[g, \phi]$ is the measure on the configuration space, and $S[g, \phi]$ is the action of the gravitational and matter fields. The two surfaces, $\Sigma_{i}$ are connected by a time-like tube and have an induced metric $h_{i}$ on them. We note that only the induced components $h_{i}$ of the full metric $g_{i}$ are independent on the surface $\Sigma_{i}[48]$.

The boundary term of the gravitational action eq. 2.50) in Chapter 2 ensures that the transition amplitude from $\Sigma_{1}$ through a surface $\Sigma_{2}$ to a third surface $\Sigma_{3}$ is obtained by summing over the fields defined on the intermediate boundary surfaces,

$$
\left\langle h_{3}, \Sigma_{3} \mid h_{1}, \Sigma_{1}\right\rangle=\sum_{h_{2}}\left\langle h_{2}, \Sigma_{2} \mid h_{1}, \Sigma_{1}\right\rangle\left\langle h_{3}, \Sigma_{3} \mid h_{2}, \Sigma_{2}\right\rangle
$$

Given the success of Wick rotation to obtain a thermal spectrum in the Schwarzschild geometry it seems not unreasonable to consider a Wick rotated gravitational path integral where, one hopes, the dominant contributions to the path integral will come from metrics and fields close to classical background fields (which extremise the action). For finite temperature these background fields (with appropriate periodicity) will have a contribution to the thermodynamic potential $W$. This has been shown to be in agreement with the thermodynamic properties of black holes, as derived by other means [21]. The Wick rotation considered in [48] is given by sending $t \rightarrow-i t$ for the coordinate along the time-like tube on the asymptotically flat boundary. This converts the Lorentzian action to the Euclidean action $S_{L} \rightarrow i S_{E}$ and the path integral is now over positive definite metrics with periodic fields,

$$
\left\langle g_{2}, \phi_{2}, \Sigma_{2} \mid g_{1}, \phi_{1}, \Sigma_{1}\right\rangle=\int D\left[g_{E}, \phi\right] \exp \left(-S_{E}\left[g_{E}, \phi\right]\right)
$$

It is now worth pointing out that the path integral approach is fraught with as many serious difficulties as any other approach to a quantisation of gravity. First of all, it is not even clear how to define such a path integral beyond a 
heuristic level, notwithstanding the problem of actually calculating anything from a well defined gravitational path integral. This is particularly so when it comes to the meaning of the measure $D[g]$ (See for instance [52, 53, 54] for discussions of this point). Secondly, it is not clear that such a Wick rotation is possible, and if it is possible, whether summing over all Euclidean signature metrics is physically equivalent to the Lorentzian problem. Furthermore, even supposing that it is possibly to Wick rotate to a physically equivalent Euclidean problem the path integral does not necessarily converge as the gravitational action is not bounded from below [48, 52]. This can be seen by considering a conformally transformed Euclidean signature metric $\tilde{g}_{a b}=$ $\Omega^{2} g_{a b}$, where $\Omega$ is a positive function fixed on the boundary. Using the transformation of the Ricci scalar, the action becomes (ignoring the boundary term),

$$
\int \tilde{R} \sqrt{\tilde{g}} \mathrm{~d}^{4} x=\int\left[\Omega^{2} R-6 \Omega^{-3}\|\nabla \Omega\|^{2}\right] \sqrt{g} \mathrm{~d}^{4} x,
$$

which can be made arbitrarily negative for rapidly changing $\Omega$.

However, for all that, it can certainly be argued that the Euclidean path integral approach has had enough success in the realm of black hole thermodynamics to merit the work and attention it has received [21, 48, 49, 50]. See also the work of Wald using an algebraic approach to Euclidean Quantum field theory in curved spacetime [55, 56]. It also provides a motivation for finding a method to Wick rotate generic Lorentzian signature metrics to Euclidean signature metrics; certainly if this method is to proceed a way to Wick rotate is needed.

\subsection{Examples of Wick Rotation}

\subsubsection{Static Metrics}

We have already seen a Wick rotation in action for the Schwarzschild metric. This can be straightforwardly generalised to arbitrary static metrics. That is, any static metric can be put into the manifestly static form [3],

$$
\mathrm{d} s^{2}=-g_{t t}\left(x^{i}\right) \mathrm{d} t^{2}+h_{i j}\left(x^{i}\right) \mathrm{d} x^{i} \mathrm{~d} x^{j}
$$


where the indices $i, j=1,2,3$ label the spatial coordinates. This form of the metric clearly admits a Wick rotation, $t \rightarrow \tau=-i t$,

$$
\mathrm{d} s^{2}=g_{t t}\left(x^{i}\right) \mathrm{d} \tau^{2}+h_{i j}\left(x^{i}\right) \mathrm{d} x^{i} \mathrm{~d} x^{j}
$$

Solutions to the vacuum equations, $R_{a b}=0$, are unchanged as the analytic continuation of zero is zero. Thus for static metrics the procedure of $t \rightarrow-i t$ is mathematically well-defined and can be employed exactly as in Minkowski space for fields defined on the curved spacetime, see for example [57]. However, even in this manifestly static case, there is a caveat that a "third law" of black hole mechanics is needed for a consistent picture. That is the surface gravity $\kappa$ must be constant over the horizon in order to complete the thermodynamic analogy.

There is another issue at play here. A static metric need not be in a manifestly static coordinate system. For example, consider the Schwarzschild solution in Painlévé-Gullstrand coordinates

$$
\mathrm{d} s^{2}=-\mathrm{d} T^{2}+\left(\sqrt{\frac{2 M}{r}} \mathrm{~d} T+\mathrm{d} r\right)^{2}+r^{2}\left(\mathrm{~d} \theta^{2}+\sin ^{2} \theta \mathrm{d} \varphi^{2}\right) .
$$

Here $\mathrm{d} T=\mathrm{d} t-(1-2 M / r)^{-1} \sqrt{2 M / r} \mathrm{~d} r$. Now sending $T \rightarrow-i T$ results in a complex valued metric. A real valued Euclidean signature metric can be found but one must also send $M \rightarrow-M$. The resultant geometry contains now a naked singularity at $r=0$ and so is somewhat pathological. It is unclear how to interpret such a result physically.

The naïve Wick rotation can be also applied to solutions of the full field equations with non-zero stress-energy tensor in manifestly static coordinates. For example the Reissner-Nordström spacetime. However the matter fields must now couple to the Euclidean signature metric.

\subsubsection{Stationary Metrics}

Turning now to the case of stationary metrics which are still independent of time but no longer irrotational, it is not entirely clear how to proceed. For 
example, such a metric can be generally put in the form [3],

$$
\mathrm{d} s^{2}=-\lambda\left(\mathrm{d} t-\omega_{j} \mathrm{~d} x^{j}\right)^{2}+\lambda^{-1} h_{i j} \mathrm{~d} x^{i} \mathrm{~d} x^{j} .
$$

Sending $t \rightarrow \tau=-i t$ here results in a complex valued metric,

$$
\mathrm{d} s^{2}=+\lambda \mathrm{d} t^{2}-2 i \lambda \omega_{j} \mathrm{~d} x^{j} \mathrm{~d} t+\lambda\left(\omega_{j} \mathrm{~d} x^{j}\right)^{2}+\lambda^{-1} h_{i j} \mathrm{~d} x^{i} \mathrm{~d} x^{j}
$$

This is clearly not a Euclidean metric and it is unclear how to immediately interpret such a result. Interestingly, the Kerr-metric, which is the unique stationary and axi-symmetric solution to the vacuum equations eq. (2.36), and represents a rotating black hole, can be manipulated to give a real valued Euclidean metric. One starts with the Kerr metric in Boyer-Lindquist coordinates [5],

$$
\mathrm{d} s^{2}=-\frac{\rho^{2} \Delta}{\Sigma} \mathrm{d} t^{2}+\frac{\Sigma}{\rho^{2}} \sin ^{2} \theta\left(\mathrm{d} \varphi-\Omega_{\text {Kerr }} \mathrm{d} t\right)^{2}+\frac{\rho^{2}}{\Delta} \mathrm{d} r^{2}+\rho^{2} \mathrm{~d} \theta^{2},
$$

where

$$
\begin{aligned}
& \rho^{2}=r^{2}+a^{2} \cos ^{2} \theta, \quad \Delta=r^{2}-2 M r+a^{2}, \\
& \Sigma=\left(r^{2}+a^{2}\right)^{2}-a^{2} \Delta \sin ^{2} \theta, \quad \Omega_{\mathrm{Kerr}} \equiv-\frac{g_{t \varphi}}{g_{\varphi \varphi}}=\frac{2 M a r}{\Sigma} \text {. }
\end{aligned}
$$

Then sending $t \rightarrow-i t$, and $a \rightarrow-i a$, results in a real valued Euclidean signature metric which satisfies the vacuum Einstein equations. This is often used to obtain the Hawking temperature by requiring the Euclidean signature solution to be periodic in the frame co-moving with the horizon. This is done to obtain a regular solution on the horizon. That is, to require the identification [21, 48]

$$
(\tau, r, \theta, \varphi)=\left(\tau+\beta, r, \theta, \varphi+i \Omega_{\mathrm{Kerr}} \beta\right)
$$

where again the inverse temperature is $\beta=2 \pi / \kappa$ and $\kappa$ is the surface gravity. It has however been argued by Brown et al. [58] that this geometry is non physical, for example it has no ergo-region, wherein, near the Kerr horizon an observer cannot remain still. They propose that a complex metric simply given by $t \rightarrow-i t$ gives a real spatial Hamiltonian and the correct ther- 
modynamic properties (They equivalently interpret the procedure as acting directly on the metric not the coordinates).

\subsubsection{Time Dependent Metrics}

The situation is even worse when considering time dependent metrics. For example following [59], the spatially flat $k=0$, de Sitter spacetime has the metric,

$$
\mathrm{d} s^{2}=-\mathrm{d} t^{2}+H^{-2} e^{H t}\left(\mathrm{~d} r^{2}+r^{2}\left(\mathrm{~d} \theta^{2}+\sin ^{2} \theta \mathrm{d} \varphi^{2}\right)\right)
$$

Sending $t \rightarrow-i t$ yields

$$
\mathrm{d} s^{2}=+\mathrm{d} t^{2}+H^{-2} e^{-i H t}\left(\mathrm{~d} r^{2}+r^{2}\left(\mathrm{~d} \theta^{2}+\sin ^{2} \theta \mathrm{d} \varphi^{2}\right)\right)
$$

This is a complex metric and has no clear physical interpretation. If instead we write de Sitter space in the co-moving coordinate with a positive spatial curvature, $k=+1$, then

$$
\mathrm{d} s^{2}=-\mathrm{d} t^{2}+H^{-2} \cosh (H t)\left(\frac{\mathrm{d} r^{2}}{1-r^{2}}+r^{2}\left(\mathrm{~d} \theta^{2}+\sin ^{2} \theta \mathrm{d} \varphi^{2}\right)\right)
$$

Now the Wick rotation $t \rightarrow-i t$ gives a Euclidean signature real valued metric,

$$
\mathrm{d} s^{2}=\mathrm{d} t^{2}+H^{-2} \cos (H t)\left(\frac{\mathrm{d} r^{2}}{1-r^{2}}+r^{2}\left(\mathrm{~d} \theta^{2}+\sin ^{2} \theta \mathrm{d} \varphi^{2}\right)\right) .
$$

This is often called the Euclidean version of de Sitter space and has nice interpretation as the metric on the hypersphere, $S^{4}$, with radius $H^{-1}$.

Hawking and Page [60] have used a naïve Wick rotation to calculate thermodynamic properties in anti-de Sitter spacetime.

This is not an exhaustive review of the situations where a naïve Wick rotation of the form $t \rightarrow-i t$ can be performed but it is an illustrative list. It is clear from the examples presented here that this form of Wick rotation specified by $t \rightarrow-i t$ is a highly coordinate dependent process and only defined in one chart. Thus for manifolds with more than one coordinate chart it cannot be applied directly. In order to preserve the structure on the manifold a generalisation of this is required. 


\subsection{Analytic Continuation of the Manifold}

The traditional method to generalise the procedure of Wick rotation when the metric is no longer independent of time, or has rotation, is to analytically continue the coordinates $x^{a} \rightarrow z^{a}$ and write the geometry in terms of a 4dimensional (4-D) complex manifold, $\mathcal{M}_{\mathbb{C}}$ [3, 48, 49]. For example extend the metric,

$$
g_{a b}(x) d x^{a} \otimes d x^{b} \rightarrow g_{a b}(z) d z^{a} \otimes d z^{b},
$$

which will satisfy the (complex) Einstein Field Equations (EFEs), c.f. eq. 2.37),

$$
G_{a b}(z)=8 \pi T_{a b}(z) .
$$

Then look for real slices of the manifold which correspond to Lorentzian and Euclidean signatures.

A manifold with complex coordinates has a natural complex structure $J$ where $J^{2}(X)=-X$ for any vector $X$ [61]. This means that an $N$ complex dimensional complex manifold can always be interpreted as an $2 N$ real dimensional manifold. Furthermore, a necessary and sufficient condition for the manifold to be a complex manifold is that the Nijenhuis tensor built from $J$ must vanish [61]. See Flaherty [62] for an overview of complex manifolds and their intersection with relativity.

Now the above route of complexification has complications on two fronts. Firstly, there is still no generic way to identify a real slice of the coordinates which corresponds to a Euclidean signature metric for arbitrary spacetimes, and where it can be done it is coordinate dependent. Secondly, when naïvely interpreted in terms of a real $8 D$ manifold this procedure gives a generically singular metric. This has been understood since the fifties, see for example Shutrick [63], but maybe it is currently overlooked.

This can be seen since the rank of $g_{a b}$ as a real metric on an $N$ dimensional manifold is $N$, and so continuing to $D=2 N$ leaves it $\operatorname{singular}$ with $\operatorname{rank}(g)=$ $N<\operatorname{dim} \mathcal{M}_{\mathbb{C}}$. Explicitly, let $z^{a}=x_{\mathrm{R}}^{a}+i x_{\mathrm{I}}^{a}$ and rewrite the arc length 
$d s^{2}=d z^{a} g_{a b}(z) d z^{b}$ in terms of real variables $x_{\mathrm{R}}^{a}$ and $x_{\mathrm{I}}^{a}$. Then we have,

$$
\begin{aligned}
d s^{2} & =\left(d x_{\mathrm{R}}^{a}+i d x_{\mathrm{I}}^{a}\right) g_{a b}\left(x_{\mathrm{R}}, x_{\mathrm{I}}\right)\left(d x_{\mathrm{R}}^{b}+i d x_{\mathrm{I}}^{b}\right) \\
& =g_{a b}\left(x_{\mathrm{R}}, x_{\mathrm{I}}\right)\left(\begin{array}{ll}
d x_{\mathrm{R}}^{a} & d x_{\mathrm{I}}^{a}
\end{array}\right)\left(\begin{array}{cc}
+1 & i \\
i & -1
\end{array}\right)\left(\begin{array}{l}
d x_{\mathrm{R}}^{b} \\
d x_{\mathrm{I}}^{b}
\end{array}\right) .
\end{aligned}
$$

Thus ordering the coordinates $x^{a}=\left(x_{\mathrm{R}}^{0} . . x_{\mathrm{R}}^{3}, x_{\mathrm{I}}^{0} \ldots x_{\mathrm{I}}^{3}\right)$ the metric becomes,

$$
\tilde{g}=g \otimes\left(\begin{array}{cc}
+1 & i \\
i & -1
\end{array}\right)
$$

which is clearly singular and therefore not invertible. Thus while it may provide a canonical isomorphism between the complexified tangent and cotangent spaces it fails when treating elements of these (i.e. vectors and covectors) as being real vectors and endowed with a real valued complex structure $J$. The question is then: how does this process have any physical meaning? It is a subtly different question to ask: if given a complex metric solution to the complex field equations eq. 6.19) can one find different real slices which correspond to Lorentzian and Euclidean signatures? However this leads to similar issues when interpreted as a real manifold endowed with complex structure.

The metric could be perhaps written non singularly if the analytic continuation transformed it into a Hermitian metric [62] in the sense,

$$
g \rightarrow g_{a \bar{b}}(z, \bar{z}) d z^{a} \otimes d \bar{z}^{b}
$$

with now $z^{a}=\frac{1}{2}\left(x_{\mathrm{R}}^{a}+i x_{\mathrm{I}}^{a}\right)$ and $\bar{z}^{a}$ its conjugate. However, there does not seem to be an obvious way to generically implement this, and the Lorentzian signature of the metric poses a barrier to introducing a Hermitian metric in the mathematicians' sense [62, 64]. Further, the additional structure of a Hermitian manifold puts strong restrictions on the Christoffel connection and hence the curvature which, while simplifying the equations nicely, seem too strong a requirement on purely physical grounds.

A compromise could potentially be reached be simply requiring the analyti- 
cally continued metric to yield real valued distances, for example,

$$
\mathrm{d} s^{2}=\frac{1}{2}\left[g_{a b}(z, \bar{z}) \mathrm{d} z^{a} \mathrm{~d} z^{b}+g_{\bar{a} \bar{b}}(z, \bar{z}) \mathrm{d} \bar{z}^{a} \mathrm{~d} \bar{z}^{b}\right]
$$

where $\overline{g_{a b}}=g_{\bar{a} \bar{b}}$. Doing this and requiring no more than analyticity of the coordinate charts and transfer functions would not impose too strong conditions on the curvature of the spacetimes. In general these extension procedures will only work for non-horizon penetrating coordinate systems. Thus even the real valued metric extension does nothing to address the coordinate dependent nature of finding a Euclidean signature slice nor does it help in the situations for which a Euclidean signature manifold admits no Lorentzian signature metric. It should, however, be noted that there have been some successes with the traditional complex extension method, see for example [48, 49, 65, 66, 67], and the references therein.

We have seen that it is not at all clear that by going to a complex manifold there is a well defined, let alone unique, way of finding Euclidean slice corresponding to a Lorentzian metric. It is the author's opinion that a different route is needed if a generalised Wick rotation compatible with general relativity is to be found.

\subsection{Analytic Continuation of the Metric}

The previous two sections have demonstrated that in a curved spacetime analytic continuation of the coordinates to a complex manifold does not directly lead to a Euclidean section. To consider an alternative approach we first return to the Feynman propagator in Minkowski space eq. (3.28),

$$
D_{\mathrm{F}}(p)=\frac{-i}{p^{2}+m^{2}-i \epsilon}=\frac{i}{\omega^{2}-k^{2}-m^{2}+i \epsilon} .
$$

Following [59] and using the infinitesimal property of $\epsilon$, the propagator can be rewritten as

$$
D_{\mathrm{F}}(p)=\frac{i}{\omega^{2}(1+i \epsilon)-\mathbf{p}^{2}-m^{2}} .
$$


Here the poles are still found at

$$
\omega= \pm \sqrt{\left(m^{2}+k^{2}\right) /(1+i \epsilon)}= \pm \sqrt{\omega_{\mathbf{k}}-i \epsilon}= \pm\left(\omega_{\mathbf{k}}-i \epsilon\right)
$$

Eq. 6.24 can now be reinterpreted in terms of a deformed Minkowski metric,

$$
\eta_{\epsilon}=\operatorname{diag}(-1-i \epsilon,+1,+1,+1)
$$

With this, the Feynman propagator can be rewritten,

$$
D_{\mathrm{F}}(p)=\frac{-i}{\eta_{\epsilon}(p, p)+m^{2}}
$$

From here, there is an obvious alternative prescription to Wick rotate to a Euclidean signature metric. That is, to analytically continue from $\epsilon=0$ to $\epsilon=2 i$ with $\operatorname{Re}(\epsilon)>0$ and avoiding the singular metric at $\epsilon=+i$. For example let $\epsilon=i\left(1-e^{i \theta}\right), \theta \in[0, \pi]$. Thus for $\eta_{\epsilon=0}=\eta_{L}$ we have the ordinary Minkowski metric and for $\eta_{\epsilon=2 i}=\eta_{E}=\operatorname{diag}(+1,+1,+1,+1)$ we have the Euclidean metric on $\mathbb{R}^{4}$, and the rotated propagator becomes,

$$
\begin{aligned}
D_{E}(x) & =\int \frac{\mathrm{d}^{4} p}{(2 \pi)^{4}} \frac{-1}{\eta_{E}(p, p)+m^{2}} \exp \left(i p^{a} \eta_{a b}^{E} x^{a}\right) \\
& =\int \frac{\mathrm{d}^{4} p}{(2 \pi)^{4}} \frac{-1}{\omega^{2}+k^{2}+m^{2}} e^{i(\omega t+\mathbf{k} \cdot \mathbf{x})} .
\end{aligned}
$$

We further note that in this procedure the determinant is $\sqrt{-\operatorname{det}\left(\eta_{\epsilon}\right)}=$ $\sqrt{1+i \epsilon}$, and so $\sqrt{-\eta_{E}}=-i=-i \sqrt{\operatorname{det}\left(\eta_{E}\right)}$. Hence continuing the metric is entirely equivalent to continuing the coordinates in Minkowski space. An equivalent formulation to this continuation of the metric has found success in continuing the Dirac spinors of fermions in Minkowski space [68]. Further this alternative Wick rotation has received attention as a possible "dynamical angle" which becomes an additional degree of freedom in a Quantum Field Theory 69.

\subsubsection{Extension to Curved Spacetime}

To answer how to extend this method to curved spacetime consider now the constant time-like vector $V=(1,0,0,0)$. Using this the deformed metric 
may be written,

$$
\eta_{\epsilon}=\eta_{L}+i \epsilon \frac{V \otimes V}{\eta_{L}(V, V)}
$$

This is a coordinate independent expression and thus has a natural extension to a curved spacetime. However it requires an additional structure, namely the existence of a time-like vector, $V$, which is defined everywhere on the manifold.

At this point it is worth taking a detour to discuss the justification for, and consequences of assuming additional structure. For reference we refer the reader to Hawking and Ellis [14], a nice exposition by Geroch and Horowitz [70] and, more recently, for a physically motived view [64]. Firstly, every manifold (we assume that a paracompactness or equivalent condition is in the definition) admits a positive definite (Euclidean signature) metric. This is not true of physical Lorentzian signature metrics which require the additional structure of a direction field. This is equivalent to being able to assign a time-like direction at each point $p$ on the manifold. These time-like vectors at $p$ can be either future- or past-directed; the distinction corresponds to a choice of direction of the arrow of time.

One can ask if the designation of future-directed vectors can be made smoothly over the manifold. If this can be done the manifold is said to be time orientable (as mentioned in Chapter 2). This is a different condition to orientability which need not make explicit reference to a metric (of any signature). Similar statements can be made about the spatial directions being space orientable. Time orientability further implies the existence of an everywhere non zero time-like vector field, which is the requirement for the generalising of the Wick rotation of the metric presented above,

$$
g_{\epsilon}=g_{L}+i \epsilon \frac{V \otimes V}{g_{L}(V, V)} .
$$

where $g_{L}=g_{a b}^{L} \mathrm{~d} x^{a} \otimes \mathrm{d} x^{b}$ is a generic solution to the Einstein equations. In components this is,

$$
\begin{aligned}
& g_{a b}^{\epsilon}=g_{a b}^{L}+i \epsilon \frac{V_{a} V_{b}}{g_{L}^{a b} V_{a} V_{b}}, \\
& g_{\epsilon}^{a b}=g_{L}^{a b}-\frac{i \epsilon}{1+i \epsilon} \frac{V^{a} V^{b}}{g_{L}^{a b} V_{a} V_{b}}
\end{aligned}
$$


where $V^{a}=g_{L}^{a b} V_{b}$. It should be pointed out that this generalisation is highly non unique and depends on the specific choice of the time-like vector field. It certainly seems natural to require that there is a least one way to consistently label time over the manifold and without any experimental evidence to the contrary we adopt this as a pragmatic approach.

Given the success of the Hamiltonian formalism in other fields of physics it makes sense to try and construct this for the gravitational Lagrangian. This is called the Arnowitt, Deser and Misner (ADM) formalism [71]. For this, it is required that spacetime be foliated by space-like hypersurfaces at least in a local patch. This foliation implies the existence of a scalar function, $\tau$, whose gradient is time-like and proportional to the unit normal of the hypersurfaces $\partial_{a} \tau \propto n_{a}$. The function $\tau$ is monotonically increasing and labels the foliating hypersurfaces $\Sigma_{\tau}$. It also provides a natural way to define a time ordering as in Minkowski space,

$$
T\left\{\hat{\phi}\left(x_{1}\right) \hat{\phi}\left(x_{2}\right)\right\}=\Theta\left(\tau_{1}-\tau_{2}\right) \hat{\phi}\left(x_{1}\right) \hat{\phi}\left(x_{2}\right)-\Theta\left(\tau_{2}-\tau_{1}\right) \hat{\phi}\left(x_{2}\right) \hat{\phi}\left(x_{1}\right)
$$

Again there is no experimental evidence to prevent this being a global assignment. This assignment leads to a time orientable manifold that is globally causally stable and effectively gives rise to the topological decomposition of the manifold into time $\times$ space, $\mathcal{M}=\mathbb{R} \times \Sigma$. This is known as the $(3+1)$ split.

The success of parity and time reversal operators in quantum mechanics suggests that spacetime be at the very least quasi-locally time and space orientable and there is no experimental reason not to extend this to a global property. This leads to further decomposition into a globally defined orthonormal tetrad $e_{A}^{a}$, where $a$ labels the spacetime coordinates and $A$ labels the individual vectors. This tetrad leads to the decomposition of the metric,

$$
g_{a b}^{L}=e_{a}^{A} e_{b}^{B} \eta_{A B}
$$

where $\eta_{A B}=\operatorname{diag}(-1,+1,+1,+1)$ is the Minkowski metric and is used to raise/lower the labels $A$. We also point out that the tetrad formalism is needed to even define the spinors of fermions and write down the Dirac 
equation in curved spacetime,

$$
\left(i \gamma^{a} \nabla_{a}-m\right) \Psi=0
$$

where the gamma matrices are defined locally by,

$$
\gamma^{a}=e_{A}^{a} \gamma^{A}, \quad\left\{\gamma^{A}, \gamma^{B}\right\}=2 \eta^{A B}
$$

In the orthonormal tetrad the analytic continuation of the metric takes the form,

$$
e_{a}^{0} e_{b}^{0} \rightarrow(1+i \epsilon) e_{a}^{0} e_{b}^{0}
$$

which in the limit $\epsilon \rightarrow 2 i$ can be reinterpreted as rotating in the (co-)tangent space $e_{a}^{0} \rightarrow-i e_{a}^{0}$. Wick rotations equivalent to this have received attention in the area of causal dynamical triangulations as a route to quantising spacetime [72, 73]. They have also been used in a proof relating to Hadamard coefficients [74].

If instead one uses a null tetrad $n_{a}^{A}$ constructed from the orthonormal tetrad, where

$$
n_{a}^{0}=\frac{1}{\sqrt{2}}\left(e_{a}^{0}+e_{a}^{1}\right), \quad n_{a}^{1}=\frac{1}{\sqrt{2}}\left(e_{a}^{0}-e_{a}^{1}\right),
$$

and $n_{a}^{2}=e_{a}^{2}, n_{a}^{3}=e_{a}^{3}$. The metric can be written

$$
g_{a b}=n_{a}^{A} n_{b}^{B} \tilde{\eta}_{A B}
$$

Here the Minkowski metric is in null form,

$$
\tilde{\eta}_{A B}=\left(\begin{array}{cc}
0 & -1 \\
-1 & 0
\end{array}\right) \bigoplus\left(\begin{array}{ll}
1 & 0 \\
0 & 1
\end{array}\right)
$$

In this case the analytic continuation of the metric takes the form

$$
\tilde{\eta}_{A B}^{\epsilon}=\left(\begin{array}{cc}
-\frac{1}{2} i \epsilon & -1-\frac{1}{2} i \epsilon \\
-1-\frac{1}{2} i \epsilon & -\frac{1}{2} i \epsilon
\end{array}\right) \bigoplus\left(\begin{array}{ll}
1 & 0 \\
0 & 1
\end{array}\right)
$$

In this limit $\epsilon \rightarrow 2 i$ this becomes

$$
\eta_{A B}^{E}=\operatorname{diag}(+1,+1,+1,+1)
$$


which is the Euclidean metric on $\mathbb{R}^{4}$.

Henceforth, we consider spacetime to be globally of the form $\mathcal{M}=\mathbb{R} \times \Sigma$, with time-like vector field $\partial_{a} \tau$ and at least one orthonormal tetrad $e_{a}^{A}$, where $e_{a}^{0} \propto \partial_{a} \tau$.

\subsubsection{Propagators}

The initial success of analytically continuing the metric can be seen in the work of Candelas and Raine [75, 76]. In the first paper they consider a static manifold with a Klein-Gordon scalar field and an equivalent prescription to eq. 6.30),

$$
g_{\lambda}=g_{L}+\lambda \frac{V \otimes V}{g_{L}(V, V)}
$$

where $V=\partial_{t}$ is the time-like Killing vector field. The operator $L_{\lambda}=$ $-\square_{\lambda}+m^{2}$ is elliptic for $\lambda>1$ and has a unique Green function $G_{\lambda}$ for the appropriate boundary conditions; $G_{\lambda} \rightarrow 0$ as $t \rightarrow \pm \infty$. This $G_{\lambda}$ is analytic as a function of $\lambda$ and by continuing to $\lambda=0$ through the upper half-plane the Feynman propagator for the physical metric is obtained.

The second paper considers a generic manifold in the causally stable form $\mathcal{M}=\mathbb{R} \times \Sigma$. They consider the same prescription as above eq. (6.43), but now $V$ is any non vanishing time-like vector field. Where applicable, the Feynman propagator is found to be obtained in the limit $\lambda \rightarrow 0^{+}$in the complex plane. However the uniqueness of this limit for two time-like vectors $V$ and $W$,

$$
\lim _{\lambda \rightarrow 0^{+}}\left[G_{\lambda}\left(x, x^{\prime} ; V\right)-G_{\lambda}\left(x, x^{\prime} ; W\right)\right]=0,
$$

is only established with the restrictions that either $V$ and $W$ differ only on a compact set or that the manifold is analytic and rays passing through $x^{\prime}$ can be extended avoiding caustics (see [76] for details).

Even with the restrictions these are suggestive results. However, much of the success of Wick Rotation in curved spacetimes is in an alternative derivation of the Hawking temperature. In the next section we show that continuation of the metric can be used to obtain the Hawking temperature. 


\subsubsection{Hawking Radiation via Metric Continuation}

Certainly for the Schwarzschild spacetime in usual coordinates, eq. 2.40, analytic continuation of the metric leads to the same Euclidean metric outside the horizon. To see this, one can take the time-like Killing vector, $V=\partial_{t}$, in which case the Euclidean metric becomes,

$$
g_{E}=g_{\text {Schwarz }}+2 \frac{\partial_{t} \otimes \partial_{t}}{\left|g^{t t}\right|}
$$

In components this is exactly eq. 4.45

$$
\mathrm{d} s^{2}=\left(1-\frac{2 M}{r}\right) \mathrm{d} \tau^{2}+\left(1-\frac{2 M}{r}\right)^{-1} \mathrm{~d} r^{2}+r^{2}\left(\mathrm{~d} \theta^{2}+\sin ^{2} \theta \mathrm{d} \varphi^{2}\right)
$$

and so requiring again that the continued metric be non-singular leads to the Hawking temperature exactly as before in Chapter 4. Further it is a simple exercise using eq. 6.5 to show that the continuation of a static metric using the time-like Killing vector in these coordinates is equivalent to the rotation $t \rightarrow-i t$.

We now turn to the case of non static metrics. Recently it has been proposed by Samuel [77] that a "Wick rotation in the tangent space" will yield the correct Hawking temperature for spacetimes with horizons, provided that the Wick rotated holonomy of the null generators is trivial. We now explain what this is saying.

Samuel considers a Wick rotation in the (orthonormal) tetrad description, $e_{a}^{0} \rightarrow i e_{a}^{0}$, where again the index $a$ labels the manifold coordinates and $A$ labels the vectors of the tetrad. Using Cartan's formalism [4], the Christoffel connection/covariant derivative $\nabla$ can be treated as a matrix valued 1-form $\Omega_{B}^{A}=e_{a}^{A}\left(\nabla_{c} e_{B}^{a}\right) \mathrm{d} x^{c}$, which is defined by Cartan's structure equation,

$$
\mathrm{d} e^{A}+\Omega_{B}^{A} \wedge e^{B}=0
$$

where $e^{A}=e_{a}^{A} \mathrm{~d} x^{a}$ and $\mathrm{d}$ is the exterior derivative. The holonomy around a closed curve $\gamma$, defined by,

$$
H_{N}=\mathcal{P} \exp \left(\int_{\gamma} \Omega_{B c}^{A} n^{c}\right)
$$


where $n^{c}$ is a tangent vector to the curve $\gamma$, represents the failure of parallel transportation along $\gamma$ to return a vector to its original orientation.

Samuel demands that the Wick rotated holonomy be trivial for the null generators of the horizon, that is $H_{N}=\exp (2 \pi J)=1$, where $J$ is a generator of $S O(4)$. This is equivalent requiring the continued metric to be non-singular on the horizon. In Chapter 4 , this forced the metric to be periodic and thus lead to a thermal spectrum with Hawking temperature.

Requiring the holonomy of the continued metric to be trivial provides a nice calculational tool for obtaining the periodicity requirements for the continuation of the metric. The "Wick rotation in the tangent space" procedure is shown to work for the Schwarzschild spacetime in the horizon penetrating Painlévé-Gullstrand coordinates [77] for which ordinary coordinate Wick rotation fails. Furthermore it is demonstrated that analytic continuation of the metric yields the Hawking temperature for the Kerr spacetime.

This is suggestive that the following is equivalent to the demand of a trivial Wick rotated holonomy on the horizon. Consider spacetimes which have a time-like vector, $\xi$, outside the horizon that becomes a null generator of the horizon. For the Euclidean metric obtained using $\xi$ (by eq. 6.30) to be non singular on the horizon, it must be periodic with period $\beta=2 \pi / \kappa$, where again $\kappa$ is the surface gravity on the horizon. That is,

$$
g_{a b}\left(x^{a}+\beta \xi^{a}\right)=g_{a b}\left(x^{a}\right)
$$

Or equivalently, identify the points $\left(x^{a}+\beta \xi^{a}\right)=\left(x^{a}\right)$ exactly as required to obtain the Hawking temperature via Euclidean methods. We have not proven this equivalence.

It is clear then, given its coordinate independent nature, that continuation of the metric is not only applicable in the same situations as a continuation of the coordinates but also to a much wider class of geometries. Further, the Euclidean signature metrics produced, while non unique, are obtained directly from a corresponding Lorentzian signature metric.

There is however a major draw back from this method. The resulting metrics are not necessarily solutions to the Euclidean Einstein equations, e.g. $R_{a b}\left(g_{E}\right)=0$. This is shown below. For notational simplicity, consider the 
time-like vectors to satisfy the normalization condition, $g_{L}^{a b} V_{b} V_{a}=-1$. The resulting identity

$$
V^{a} \nabla_{b}^{L} V_{a}=0
$$

is used extensively in the following. To evaluate the rotated Ricci tensor in terms of the original metric we need to relate the two affine connexions.

$$
\begin{aligned}
{\left[\Gamma_{\varepsilon}\right]_{b c}^{a} \equiv } & \frac{1}{2} g_{\varepsilon}^{a m}\left(g_{m b, c}^{\varepsilon}+g_{m c, b}^{\varepsilon}-g_{c b, m}^{\varepsilon}\right) \\
= & \frac{1}{2}\left(g_{L}^{a m}+\frac{i \varepsilon}{1+i \varepsilon} V^{a} V^{m}\right)\left(\partial_{c}\left[g_{m b}^{L}-i \varepsilon V_{m} V_{b}\right]+\partial_{b}\left[g_{m c}^{L}-i \varepsilon V_{m} V_{c}\right]\right. \\
& \left.+\partial_{m}\left[g_{b c}^{L}-i \varepsilon V_{b} V_{c}\right]\right) .
\end{aligned}
$$

This can be written,

$$
\left[\Gamma_{\epsilon}\right]_{b c}^{a}=\left[\Gamma_{L}\right]_{b c}^{a}-Z_{b c}^{a}
$$

where we introduce the tensor,

$$
\begin{aligned}
Z_{b c}^{a} & =i \epsilon\left(V_{(b} \nabla_{c)}^{L} V^{a}-V_{(b} \nabla_{L}^{a} V_{c)}\right)+\frac{i \epsilon}{1+i \epsilon} V^{a} \nabla_{(c}^{L} V_{b)}+\frac{\epsilon^{2}}{1+i \epsilon} V^{a} V_{(b} A_{c)} \\
& =i \epsilon V_{(b} F_{c)}^{a}+\frac{i \epsilon}{2(1+i \epsilon)} V^{a} Q_{b c}+\frac{\epsilon^{2}}{1+i \epsilon} V^{a} V_{(b} A_{c)}
\end{aligned}
$$

where $\nabla^{L}$ denotes the covariant derivative with respect to the Lorentzian signature metric, $F_{a b}=2 V_{[a ; b]}, Q_{a b}=2 V_{(a ; b)}$, and $A^{a}=V^{m} \nabla_{m}^{L} V^{a}$. It should be noted that $Z_{b c}^{a}$ can be written equivalently in terms of $g_{\epsilon}$ and the Christoffel connection defined by $g_{\epsilon}$. A small computation shows $Z_{b a}^{a}=0$ and by definition $Z_{[b c]}^{a}=0$.

Now invoking the $(3+1)$ decomposition, $V_{a}=-\mu \partial_{a} \tau$ for a normalisation factor $\mu$, and is hypersurface orthogonal. Using Frobenius' theorem this implies $V_{[a ; b} V_{c]}=0$ [5]. From this one can show that,

$$
F_{a b}=A_{b} V_{a}-A_{b} V_{a}
$$

Substitution of eq. 6.53 into eq. 6.52 leads to

$$
Z_{b c}^{a}=\frac{i \epsilon}{1+i \epsilon} V^{a}\left(V_{(b} A_{c)}+\frac{1}{2} Q_{b c}\right) .
$$

In the case that $V^{a}=-\mu \partial_{a} \tau$ satisfies the affinely parametrised geodesic 
equation $A_{a}=0$ then eq. (6.54) simplifies further

$$
Z_{b c}^{a}=\frac{i \epsilon}{1+i \epsilon} V^{a} \nabla_{b} V_{c}
$$

Now using eq. 6.51) the rotated Riemann and Ricci tensors can be explicitly related to the original tensors. That is,

$$
\begin{aligned}
{\left[R_{\varepsilon}\right]_{b c d}^{a} } & =\left[R_{L}\right]_{b c d}^{a}+2\left(Z_{b[c ; d]}^{a}+Z_{n[d}^{a} Z_{|b| c]}^{n}\right), \\
R_{a b}^{\varepsilon} & =R_{a b}^{L}+2\left(Z_{a[c ; b]}^{c}+Z_{n[b}^{c} Z_{|a| c]}^{n}\right),
\end{aligned}
$$

where $; \equiv \nabla^{L}$. Eq. 6.56b can be simplified using the symmetries of $Z_{b c}^{a}$ above,

$$
R_{a b}^{\varepsilon}=R_{a b}^{L}-\nabla_{c}^{L} Z_{a b}^{c}+Z_{n b}^{c} Z_{a c}^{n}
$$

This shows that an analytic continuation of the metric result in spacetimes which are solutions to the traditional vacuum Euclidean Einstein equations $R\left(g_{E}\right)_{a b}=0$ iff

$$
\nabla_{c}^{L} Z_{a b}^{c}=Z_{n b}^{c} Z_{a c}^{n}
$$

This can be simplified using eq. (6.54) to

$$
\nabla_{m}\left(V^{m}\left[V_{(a} A_{b)}+\frac{1}{2} Q_{a b}\right]\right)=\frac{i \epsilon}{1+i \epsilon} A_{a} A_{b} .
$$

\subsubsection{A Modified Action?}

We have seen that there are suggestive results, for both propagators and the Hawking temperature from, an analytic continuation of the metric but that the continued metric is no longer a solution to the same vacuum equations. This suggests that in order to write down a Wick rotated path integral which incorporates the metrics obtained by continuation as the stationary points one cannot simply take $\sqrt{-g_{\epsilon}} R\left(g_{\epsilon}\right)$ as the Wick rotated Lagrangian density. Barbero [78] has previously introduced a modified action for essentially the Wick rotation of the metric we use. Following [78, 79] and working again with the spacetime decomposition $\mathcal{M}=\mathbb{R} \times \Sigma$, equipped with time-like vector field $V_{a}=\partial_{a} \tau$, where $\tau$ labels the foliation of space-like hypersurfaces $\Sigma_{\tau}$, we consider the Lorentzian signature metric as a function of a Euclidean 
signature one,

$$
\begin{aligned}
g_{a b}^{L} & =g_{a b}^{\epsilon}+i \epsilon \frac{V_{a} V_{b}}{\left|g_{\epsilon}^{m n} V_{m} V_{n}\right|}, \\
g_{L}^{a b} & =g_{\epsilon}^{a b}-\frac{i \epsilon}{1+i \epsilon} \frac{V^{a} V^{b}}{\left|g_{\epsilon}^{a b} V_{a} V_{b}\right|},
\end{aligned}
$$

where now $V^{a}=g_{\epsilon}^{a b} V_{b}$ and we have used $\left|g_{L}^{m n} V_{m} V_{n}\right|=\left|g_{\epsilon}^{m n} V_{m} V_{n}\right|$, for $\mid 1+$ $i \epsilon \mid=1$ where $\epsilon$ is complex. This is just the inverse of the Wick rotation eq. (6.30).

Further considering the Wick rotated Lagrangian density to be $\sqrt{-g_{\epsilon}} R\left[g_{L}\left(g_{\epsilon}\right)\right]$, a long computation using eq. (6.57) and discarding surface terms shows that the action can be written

$$
\begin{aligned}
S\left[g_{\epsilon}\right] & =\int\left(R\left(g_{\epsilon}\right)-\frac{i \epsilon}{1+i \epsilon} R_{a b}\left(g_{\epsilon}\right) \frac{V_{a} V_{b}}{\left|g_{\epsilon}^{m n} V_{m} V_{n}\right|}\right) \sqrt{-g_{\epsilon}} \mathrm{d}^{4} x, \\
& =\int\left(R\left(g_{\epsilon}\right)-\frac{i \epsilon}{1+i \epsilon} R_{a b}\left(g_{\epsilon}\right) U^{a} U^{b}\right) \sqrt{-g_{\epsilon}} \mathrm{d}^{4} x,
\end{aligned}
$$

where $U_{a}=\left|\left(g_{\epsilon}\right)^{m n} V_{m} V_{n}\right|^{-1 / 2} V_{a}$. The vector $U_{a} \propto \partial_{a} \tau$ is again hypersurface orthogonal by virtue of the manifold decomposition and so once more satisfies Frobenius' theorem [5].

For $\epsilon=0$ this is clearly the ordinary Lorentzian action eq. 2.50 , for $\epsilon=2 i$ we obtain a Euclidean action,

$$
S\left[g_{E}\right]=-i \lim _{\epsilon \rightarrow 2 i} S\left[g_{\epsilon}\right]=\int\left(R\left(g_{E}\right)-2 R_{a b}\left(g_{E}\right) \frac{V_{a} V_{b}}{\left|g_{E}^{m n} V_{m} V_{n}\right|}\right) \sqrt{g_{E}} \mathrm{~d}^{4} x
$$

taking $\sqrt{-g_{E}}=i \sqrt{g_{E}}$.

Now using the techniques of disformal transformations [80] it can be shown that, for all values of $\epsilon$, the new action eq. $(6.62)$ has the same extrema as the action for Lorentzian signature gravity eq. (2.50). Moreover demanding the action to be extremal with respect to the field $\tau$ produces no new constraints on the field equations. Therefore one can consider this action as a functional of only the continued metric $g_{\epsilon}$ for the path integral approach, safe in the knowledge that one can consistently use the Wick rotation of the metric to switch between Euclidean and Lorentzian signature.

Thus we can consider the thermal partition function for the Euclidean theory 
to be defined by

$$
Z_{\mathrm{G}}=\int D\left[g_{L}, \tau, \phi\right] \exp \left(-S_{E}\left[g_{E}, \phi\right]\right)
$$

where $S_{E}\left[g_{E}\right]$ is determined by eq. (6.63) and taken to be over fields periodic in the vector $V$ with period $\beta$. The measure, $D\left[g_{L}, \tau, \phi\right]$, now only runs over the physically relevant Lorentzian signature metrics, and does not include those Euclidean signature geometries which admit no Lorentzian one. However, one has to take into account the vector field $V_{a}=\partial_{a} \tau$. Since the extrema of the action are independent of $V$ it seems plausible that this introduces no more than a phase factor. Moreover, the stationary phase approximations to thermodynamic properties around background Euclidean continued metrics can be consistently be found via the new action.

\subsection{Open Problems and Further Work}

In this chapter we have summarised the many open problems for generalising Wick rotation to curved spacetime, and presented some alternative lines for future work. It has been shown that the traditional $t \rightarrow-i t$ fails in general. Further we have presented the difficulties for extending the real manifold to a complex manifold $\mathcal{M}_{\mathbb{C}}$ with complex metric. Namely that the naïvely defined metric is singular and there is no generic way to find real slices which correspond to different signatures. However, we have proposed a route via a complex extension to a real-valued non singular metric, eq. 6.23, that could potentially lead to a more well-defined method of looking for real slices of $\mathcal{M}_{\mathbb{C}}$. Nevertheless, this does not address the coordinate and chart dependency of the technique.

We also gave an exposition of analytic continuation of the metric, eq. 6.30, as an alternative to continuing the coordinates. Using the $(3+1)$ decomposition the continued metric was shown to have success in both defining Feynman propagators and leading to the Hawking temperature. However in order to consistently switch between the Euclidean and Lorentzian picture via this method it was necessary to introduce a new action, eq. 6.62). This action allows for the Euclidean path integral to be written down consistently and hence the corresponding thermal properties to be found using the Eu- 
clidean techniques of Chapter 3. Further this path integral is now over only the physically relevant Lorentzian signature geometries.

There are also a number of suggestive lines of further work to be pursued involving continuation of the metric. It is certainly desirable to try to show that the resulting geometries produce the same physical properties independent of the vector used to continue the metric. There is also scope develop the work of Candelas and Raine [75, 76] for propagators in curved spacetime. Further, in some ways the tetrad formalism is as fundamental as the metric and is certainly required to deal with fermions in curved spacetime. It would therefore be interesting to try and extend the work of Samuel [77] by looking at the Wick rotated action in the tetrad formalism and relate this to eq. 6.62). It would also be of interest to rigorously convert the statements made about Holonomy into statements about the periodicity of the continued Euclidean metric.

On the path integral side, understanding the new measure $D[g, \tau]$ is of crucial importance to try to continue down the Euclidean quantum gravity route. It would also be very worthwhile to calculate the new action under a conformal transformation $\tilde{g}_{a b}^{E}=\Omega^{2} g_{a b}^{E}$ and see whether it is still unbounded from below and whether this can be accounted for in the measure. Finally, invoking a $(3+1)$ split is reminiscent of the Wheeler-DeWitt equation and canonical quantum gravity [81. It would be interesting to write the new action eq. 6.62) in terms of the spatial Ricci scalar and extrinsic curvature along the lines of Barbero [78] and find the resulting Wheeler-DeWitt equation following 82 .

It is the author's opinion that the most promising outlook for a generalised Wick rotation will be along these directions for a continued metric and not for the continuation of the coordinates. 


\section{Chapter 7}

\section{Conclusions}

In this thesis we have explored the interaction between general relativity and quantum field theory, focussing on the Hawking radiation effect for black holes. The study consisted of two main themes. The first looked at the greybody factors arising in the Hawking effect from the gravitational potential. The second investigated the extension of Wick rotation to curved spacetime, motivated by the Euclidean derivation of the Hawking effect and Euclidean quantum gravity.

We have developed a new method for numerical calculation of the greybody factors which has wider application to other one dimensional scattering problems. Although we have only applied it to spherically symmetric black holes there is certainly scope to apply the scheme to the more general cases. The effect of the greybody factors on the Hawking process is that they significantly reduce the emission rate for Schwarzschild black holes and consequently Hawking radiation is extremely sparse. This provides hints about how to re-interpret the process as a chain of two-body decays [1].

Difficulties arise when looking at Reissner-Nordström, Kerr, and Kerr-Newman black holes. In these situations one must account for super-radiance due to the interplay between the greybody factors and the change of sign of the Bose occupation number. This can lead to greatly enhanced emission rates [23, 35, 36]. The product calculus method could be extended to these situations taking into account the Teukolsky equation [37]. Further there is room to gain a better understanding of all greybody situations using semianalytic approximations via the Dyson/Peano series eq. 5.52. 
On the Wick rotation front we have seen that $t \rightarrow-i t$ does not naturally extend, as is, to non static spacetimes. There are certain situations where a Wick rotation, $t \rightarrow-i t$, can be applied, and used to find thermodynamic properties, however it is highly coordinate dependent. Further, when considering complex valued metrics the extension to complex manifolds is not well defined. We have introduced a way to extend to a complex manifold using a real valued metric although this is still a coordinate dependent process which is not cleanly generalisable.

On the other hand it seems that an analytic continuation of the metric works in all the situations where a continuation of the coordinates works. Further it is coordinate independent and can be applied to any Lorentzian metric in a time orientable manifold. In spite of its successes, the analytic continuation of the metric requires a new action, $S_{E}$ in eq. (6.63). This is necessary in order to have the extrema $\delta S_{E}=0$ occur at the Euclidean signature metrics obtained via continuation from the physically relevant Lorentzian signature ones.

There is plenty of further work to be done to develop the ideas associated with analytic continuation of the metric. This should focus on examining the dependence of the process on the time-like vector employed along the lines of Candelas and Raine [76], and understanding the relation between the new proposed action and the tetrad formalism employed by Samuel [77]. Finally special attention needs to be paid to understanding the measure, $D[g, \tau]$, of the Wick rotated action and relating it to the Lorentzian measure.

Ultimately the success of any extension of Wick rotation will depend on a deeper understanding of the nature of time in general relativity and the path integral approach to a quantum gravity. 


\section{Bibliography}

[1] Finnian Gray, Sebastian Schuster, Alexander Van-Brunt, and Matt Visser. The hawking cascade from a black hole is extremely sparse. Classical and Quantum Gravity, 33(11):115003, 2016.

[2] Finnian Gray and Matt Visser. Greybody factors for Schwarzschild black holes: Path-ordered exponentials and product integrals. preprint, arXiv:1512.05018, 2015.

[3] Robert M. Wald. General Relativity. University of Chicago Press, 1984.

[4] Charles W. Misner, Kip S. Thorne, and John A. Wheeler. Gravitation. W. H. Freeman, 1973.

[5] Eric Poisson. A Relativist's Toolkit: The Mathematics of Black-Hole Mechanics. Cambridge University Press, 2004.

[6] John A. Wheeler and Kenneth Ford. Geons, Black Holes, and Quantum Foam: A Life in Physics. W. W. Norton, 2000.

[7] Nicholas D. Birrell and Paul C. W. Davies. Quantum Fields in Curved Space. Cambridge Monographs on Mathematical Physics. Cambridge University Press, 1984.

[8] Michael E. Peskin and David V. Schroeder. An Introduction To Quantum Field Theory. Frontiers in physics. Westview Press, 1995.

[9] Richard P. Feynman and Albert R. Hibbs. Quantum Mechanics and Path Integrals. Dover books on physics. Dover Publications, 2010.

[10] Konrad Osterwalder and Robert Schrader. Axioms for Euclidean Green's functions II. Communications in Mathematical Physics, 42(3):281-305, 1975. 
[11] Ryogo Kubo. Statistical-Mechanical Theory of Irreversible Processes. I. General Theory and Simple Applications to Magnetic and Conduction Problems. Journal of the Physical Society of Japan, 12(6):570-586, 1957.

[12] Paul C. Martin and Julian Schwinger. Theory of many-particle systems. i. Phys. Rev., 115:1342-1373, Sep 1959.

[13] Stephen W. Hawking. Particle creation by black holes. Communications in Mathematical Physics, 43(3):199-220, 1975.

[14] Stephen W. Hawking and George F. R. Ellis. The Large Scale Structure of Space-Time. Cambridge Monographs on Mathematical Physics. Cambridge University Press, 1973.

[15] Stephen A. Fulling. Nonuniqueness of Canonical Field Quantization in Riemannian Space-Time. Phys. Rev. D, 7:2850-2862, May 1973.

[16] Paul C. W. Davies. Scalar production in Schwarzschild and Rindler metrics. Journal of Physics A, 8(4):609, 1975.

[17] William G. Unruh. Notes on black-hole evaporation. Phys. Rev. D, 14:870-892, Aug 1976.

[18] John M. Bardeen, Brandon Carter, and Stephen W. Hawking. The four laws of black hole mechanics. Communications in Mathematical Physics, 31(2):161-170, 1973.

[19] Jacob D. Bekenstein. Black holes and the second law. Nuovo Cimento, 4(15):737-740, 1972.

[20] Stephen W. Hawking. Black holes and thermodynamics. Phys. Rev. D, 13:191-197, Jan 1976.

[21] Gary W. Gibbons and Stephen W. Hawking. Action integrals and partition functions in quantum gravity. Phys. Rev. D, 15:2752-2756, May 1977.

[22] James B. Hartle and Stephen W. Hawking. Path-integral derivation of black-hole radiance. Phys. Rev. D, 13:2188-2203, Apr 1976. 
[23] Don N. Page. Particle emission rates from a black hole: Massless particles from an uncharged, nonrotating hole. Phys. Rev. D, 13:198-206, Jan 1976.

[24] Richard H. Price. Nonspherical Perturbations of Relativistic Gravitational Collapse. I. Scalar and Gravitational Perturbations. Phys. Rev. D, 5:2419-2438, May 1972.

[25] Richard H. Price. Nonspherical Perturbations of Relativistic Gravitational Collapse. II. Integer-Spin, Zero-Rest-Mass Fields. Phys. Rev. D, 5:2439-2454, May 1972.

[26] Valeri Frolov and Igor Novikov. Black Hole Physics: Basic Concepts and New Developments. Fundamental Theories of Physics. Springer Netherlands, 1998.

[27] Subrahmanyan Chandrasekhar. The Mathematical Theory of Black Holes. Oxford classic texts in the physical sciences. Clarendon Press, 1998.

[28] Hans Stephani. Debye potentials in Riemannian spaces. J. Math. Phys., 15:14-16, 1974.

[29] Dennis Philipp and Volker Perlick. On analytic solutions of wave equations in regular coordinate systems on Schwarzschild background. preprint, arXiv:1503.08101, 2015.

[30] Tse Chin Mo and Charles H. Papas. Electromagnetic Field and Wave Propagation in Gravitation. Phys. Rev. D, 3:1708-1712, Apr 1971.

[31] R. M. Corless, G.H. Gonnet, D. E. G. Hare, D. J. Jeffrey, and D. E. Knuth. On the Lambert W function. Advances in Computational Mathematics, 5(1):329-359, 1996.

[32] Tullio Regge and John A. Wheeler. Stability of a Schwarzschild Singularity. Phys. Rev. D, 108:1063-1069, Nov 1957.

[33] Frank J. Zerilli. Effective potential for even parity Regge-Wheeler gravitational perturbation equations. Phys. Rev. Lett., 24:737-738, 1970. 
[34] Plamen P. Fiziev. Exact solutions of Regge-Wheeler equation. Journal of Physics: Conference Series, 66(1):12-16, 2007.

[35] Don N. Page. Particle emission rates from a black hole. II. Massless particles from a rotating hole. Phys. Rev. D, 14:3260-3273, Dec 1976.

[36] Don N. Page. Particle emission rates from a black hole. III. Charged leptons from a nonrotating hole. Phys. Rev. D, 16:2402-2411, Oct 1977.

[37] Saul A. Teukolsky. Perturbations of a rotating black hole. 1. Fundamental equations for gravitational electromagnetic and neutrino field perturbations. Astrophys. J., 185:635-647, 1973.

[38] Alexei A. Starobinsky and Semyon M. Churilov. Amplification of electromagnetic and gravitational waves scattered by a rotating black hole. Sov. Phys. JETP, 38, 1974.

[39] Matt Visser. Some general bounds for one-dimensional scattering. Phys. Rev. A, 59:427-438, Jan 1999.

[40] Petarpa Boonserm and Matt Visser. Reformulating the Schrodinger equation as a Shabat-Zakharov system. J. Math. Phys., 51:022105, 2010.

[41] Petarpa Boonserm and Matt Visser. Bounding the greybody factors for Schwarzschild black holes. Phys. Rev. D, 78:101502, Nov 2008.

[42] Petarpa Boonserm, Tritos Ngampitipan, and Matt Visser. Bounding the greybody factors for scalar field excitations on the Kerr-Newman spacetime. Journal of High Energy Physics, 113(3):1-19, 2014.

[43] John D. Dollard and Charles N. Friedman. Product Integration with Application to Differential Equations. Encyclopedia of Mathematics and its Applications. Cambridge University Press, 1984.

[44] John D. Dollard and Charles N. Friedman. Product integration of measures and applications. Journal of Differential Equations, 31(3):418-464, 1979 .

[45] Antonín Slavk. Product Integration, Its History and Applications. Djiny Matematiky. Matfyzpress, 2007. 
[46] Jon Helton. Product integrals and the solution of integral equations. Pacific J. Math., 58(1):87-103, 1975.

[47] Jon Helton and Stephen Stuckwisch. Numerical approximation of product integrals. Journal of Mathematical Analysis and Applications, 56(2):410-437, 1976.

[48] Stephen W. Hawking. The path-integral approach to quantum gravity. In Stephen W. Hawking and Werner Israel, editors, General Relativity: An Einstein Centenary Survey. Cambridge University Press, 1979.

[49] Stephen W. Hawking. Euclidean Quantum Gravity. In Maurice Lévy and Stanley Deser, editors, Recent Developments in Gravitation, volume 44 of NATO Advanced Study Institutes Series. Springer US, 1979.

[50] Gary W. Gibbons and Stephen W. Hawking. Euclidean Quantum Gravity. World Scientific, 1993.

[51] Charles W. Misner. Feynman Quantization of General Relativity. Rev. Mod. Phys., 29:497-509, Jul 1957.

[52] Pawel O. Mazur and Emil Mottola. The path integral measure, conformal factor problem and stability of the ground state of quantum gravity. Nuclear Physics B, 341(1):187-212, 1990.

[53] Emil Mottola. Functional integration over geometries. J. Math. Phys., 36:2470-2511, 1995.

[54] Arundhati Dasgupta. The gravitational path integral and the trace of the diffeomorphisms. General Relativity and Gravitation, 43(8):2237$2255,2011$.

[55] Robert M. Wald. On the Euclidean approach to quantum field theory in curved spacetime. Communications in Mathematical Physics, 70(3):221242, 1979.

[56] Robert M. Wald. Quantum Field Theory in Curved Spacetime and Black Hole Thermodynamics. Chicago Lectures in Physics. University of Chicago Press, 1994. 
[57] Saul A. Fulling and Simon N. M. Ruijsenaars. Temperature, periodicity and horizons. Physics Reports, 152(3):135-176, 1987.

[58] J. David Brown, Erik A. Martinez, and James W. York. Complex KerrNewman geometry and black-hole thermodynamics. Phys. Rev. Lett., 66:2281-2284, May 1991.

[59] Matt Visser. How to Wick rotate generic curved spacetime. unpublished, http://www.gravityresearchfoundation.org/pdf/awarded/1991/vissar.pdf, 1991.

[60] Stephen W. Hawking and Don N. Page. Thermodynamics of black holes in anti-de Sitter space. Communications in Mathematical Physics, 87(4):577-588, 1983.

[61] Kunihiko Kodaira. Complex Manifolds and Deformation of Complex Structures. Classics in Mathematics. Springer, 2004.

[62] Edward J. Flaherty. Hermitian and Khlerian Geometry in Relativity. Lecture Notes in Physics. Springer Berlin Heidelberg, 1975.

[63] H. B. Shutrick. Complex Extensions. Quarterly Journal of Mathematics, 9:197, 1958.

[64] Deloshan Nawarajan and Matt Visser. Global properties of physically interesting Lorentzian spacetimes. preprint, arXiv:1601.03355, 2016.

[65] Nicholas Woodhouse. The Real Geometry of Complex Space-Times. International Journal of Theoretical Physics, 16(9):663-670, 1977.

[66] A. Meister. Complex manifold methods in quantum field theory in curved spacetime. J. Math. Phys., 30(12):2930-2942, 1989.

[67] Christer Helleland and Sigbjorn Hervik. A Wick-rotatable metric is purely electric. preprint, arXiv:1504.01244, 2015.

[68] Mayank R. Mehta. Euclidean continuation of the Dirac fermion. Phys. Rev. Lett., 65:1983-1986, Oct 1990.

[69] Alberto Carlini and Jeff Greensite. Why is spacetime Lorentzian? Phys. Rev. D, 49:866-878, Jan 1994. 
[70] Robert Geroch and Gary T. Horowitz. Global structure of spacetimes. In Stephen W. Hawking and Werner Israel, editors, General Relativity; an Einstein Centenary Survey. Cambridge University Press, 1979.

[71] Richard Arnowitt, Stanley Deser, and Charles W. Misner. Dynamical Structure and Definition of Energy in General Relativity. Phys. Rev., 116:1322-1330, Dec 1959.

[72] Arundhati Dasgupta. The Real Wick Rotations in Quantum Gravity. Journal of High Energy Physics, 2002(07):062, 2002.

[73] Arundhati Dasgupta and Renate Loll. A proper-time cure for the conformal sickness in quantum gravity . Nuclear Physics B, 606(12):357-379, 2001.

[74] Valter Moretti. Proof of the symmetry of the off diagonal Hadamard/Seeley-deWitt's coefficients in $C^{\infty}$ Lorentzian manifolds by a 'local Wick rotation'. Communications in Mathematical Physics, 212:165-189, 2000 .

[75] Philip Candelas and Derek J. Raine. Quantum field theory on incomplete manifolds. J. Math. Phys., 17(11):2101-2112, 1976.

[76] Philip Candelas and Derek J. Raine. Feynman propagator in curved space-time. Phys. Rev. D, 15:1494-1500, Mar 1977.

[77] Joseph Samuel. Wick Rotation in the Tangent Space. Class. Quant. Grav., 33(1):015006, 2016.

[78] J. Fernando Barbero G. From Euclidean to Lorentzian general relativity: The real way. Phys. Rev. D, 54:1492-1499, Jul 1996.

[79] J. Fernando Barbero G. and Eduardo J.S. Villaseñor. Lorentz violations and Euclidean signature metrics. Phys. Rev. D, D68:087501, 2003.

[80] Nathalie Deruelle and Josephine Rua. Disformal transformations, veiled General Relativity and Mimetic Gravity. Journal of Cosmology and Astroparticle Physics, 2014(09):002, 2014.

[81] Bryce S. DeWitt. Quantum Theory of Gravity. I. The Canonical Theory. Phys. Rev., 160:1113-1148, Aug 1967. 
[82] James B. Hartle and Stephen W. Hawking. Wave function of the Universe. Phys. Rev. D, 28:2960-2975, Dec 1983. 